\title{
QED radiative corrections to virtual Compton scattering
}

\author{
M. Vanderhaeghen ${ }^{1}$, J.M. Friedrich ${ }^{1}$, D. Lhuillier ${ }^{2}$, D. Marchand ${ }^{3}$, L. Van Hoorebeke ${ }^{4}$ and \\ J. Van de Wiele ${ }^{3}$. \\ ${ }^{1}$ Institut für Kernphysik, Johannes Gutenberg Universität, D-55099 Mainz, Germany \\ ${ }^{2}$ CEA/Saclay, DAPNIA/SPhN, F-91191 Gif-sur-Yvette, France \\ 3 Institut de Physique Nucléaire, F-91406 Orsay, France \\ ${ }^{4}$ FWO-Vlaanderen, RUG, Proeftuinstraat 86, B-9000 Gent, Belgium
}

(September 13, 2018)

\begin{abstract}
The QED radiative corrections to virtual Compton scattering (reaction $e p \rightarrow e p \gamma)$ are calculated to first order in $\alpha_{e m} \equiv e^{2} / 4 \pi$. A detailed study is presented for the one-loop virtual corrections and for the first order softphoton emission contributions. Furthermore, a full numerical calculation is given for the radiative tail, corresponding with photon emission processes, where the photon energy is not very small compared with the lepton momenta. We compare our results with existing works on elastic electron-proton scattering, and show for the $e p \rightarrow e p \gamma$ reaction how the observables are modified due to these first order QED radiative corrections. We show results for both unpolarized and polarized observables of the virtual Compton scattering in the low energy region (where one is sensitive to the generalized polarizabilities of the nucleon), as well as for the deeply virtual Compton scattering.
\end{abstract}

PACS : 13.40.Ks, 13.60.-r, 13.60.Fz 


\section{INTRODUCTION}

Virtual Compton scattering (VCS) has become in recent years a new and versatile tool in the study of nucleon structure and has triggered an important activity on both the theoretical and experimental side (see e.g. [1,2]). VCS, which is accessed through the $\left(e, e^{\prime} \gamma\right)$ reaction, is studied now in various kinematical domains.

At low energy, below pion production threshold, it allows to access generalized polarizabilities of the proton 3, 31. These response functions, which constitute new nucleon structure observables, have been calculated in various approaches and models [3,5 9]. To extract this nucleon structure information from VCS below pion production threshold, a considerable experimental effort is taking place at various electron laboratories. The first few events of VCS were observed in [10]. The first dedicated VCS experiment has been performed at MAMI and for the first time, two combinations of generalized polarizabilities have been determined at a four-momentum squared $Q^{2}=0.33 \mathrm{GeV}^{2}$ [11]. An experiment at higher $Q^{2}$ $\left(1-2 \mathrm{GeV}^{2}\right)$ at JLab [12] has already been performed, which is under analysis at the time of writing, and a further experiment at lower $Q^{2}$ is planned at MIT-Bates [13].

The VCS is also studied vigorously in the Bjorken regime (where the photon virtuality $Q^{2}$ and the photon-proton c.m. energy $\sqrt{s}$ are both large, with $Q^{2} / s$ finite), which is referred to as deeply virtual Compton scattering (DVCS). In this region, the DVCS amplitude is parametrized at leading order in $Q$ in terms of six generalized parton distributions [14 16], commonly denoted as skewed parton distributions (4 quark helicity conserving functions and 2 which involve a quark helicity flip). This field has generated by now a whole theoretical industry, and first experiments of DVCS and related hard electroproduction reactions are being performed, analyzed or planned both at JLab [17], HERMES/HERA [18], and COMPASS [19].

The first absolute measurement of the VCS cross section on the nucleon performed at MAMI [11], indicates that QED radiative corrections provide an important contribution to the $e p \rightarrow e p \gamma$ reaction (of the order of $20 \%$ in the kinematics considered in [11]). The $e p \rightarrow e p \gamma$ reaction is particular in comparison with other electron scattering reactions because the photon can be emitted from both the proton side (this is the VCS process which contains the nucleon structure information of interest) or can be emitted from one of the electrons (which is the parasitic Bethe-Heitler process). The radiative corrections to the Bethe-Heitler process are formally different compared with the case of electron scattering. The importance of a very good understanding of the radiative corrections is indispensable if one wants to extract nucleon structure information from the $e p \rightarrow e p \gamma$ reaction, especially in those kinematical situations where the Bethe-Heitler process is not negligible. The calculation of these QED radiative corrections to the $e p \rightarrow e p \gamma$ reaction to first order in $\alpha_{e m} \equiv e^{2} / 4 \pi \approx 1 / 137.036$, is the subject of this paper.

Radiative corrections were first calculated by Schwinger for potential scattering [20]. Tsai [21] extented the calculation of the radiative corrections to electron-proton scattering. The field has a long history and we refer to the standard review papers [22,23], which were used in the interpretation of many electron scattering experiments.

The outline of the present paper is as follows. In section 【1, we introduce the kinematics and notations used for the $e p \rightarrow e p \gamma$ reaction, and give the lowest order amplitudes.

In section [II, we give the first order QED radiative corrections to the $e p \rightarrow e p \gamma$ reaction. 
We first calculate, in section ШIIA, the one-loop virtual radiative corrections originating from the lepton side, to the $e p \rightarrow e p \gamma$ reaction. Our strategy used to evaluate the rather complicated loop integrals, is to solve first simpler loop integrals, which contain entirely the ultraviolet (UV) and infrared (IR) divergences, and in which the lowest order amplitudes factorize. These simpler loop integrals are evaluated analytically. The finite remainder with respect to the original amplitude, is then expressed through Feynman parameter integrals, which are calculated numerically in this work.

In section [IIB, we calculate the soft photon emission contributions from the lepton side, to the $e p \rightarrow e p \gamma$ reaction. We discuss the similarities and differences with the bremsstrahlung contribution to elastic electron-nucleon scattering. These bremsstrahlung processes contain IR divergences which are shown to cancel exactly the IR divergences from the virtual photon processes.

In section $\amalg I \mathrm{IO}$, the numerical method to evaluate the remaining finite Feynman parameter integrals is presented. We discuss subsequently the cases where the integrand is regular or singular, the latter originating from the propagation of on-shell intermediate states in the one-loop corrections to the $e p \rightarrow e p \gamma$ reaction. In particular, we discuss the different numerical checks performed and the accuracy of the calculation.

In section IIID, we discuss the radiative corrections at the proton side and the twophoton exchange corrections, by referring to the recent work of Maximon and Tjon [24].

In section $\mathbb{\square}$, we give a full numerical calculation for elastic electron-proton scattering of the photon emission processes where the photon energy is not very small compared with the lepton momenta, and which makes up the radiative tail. We compare this full calculation with an approximate procedure based on the angular peaking approximation, and show to what extent the full calculation validates the approximate method for the case of elastic electron-nucleon scattering. The approximate method will be seen to be realistic enough to apply it next to the calculation of the radiative tail in the case of VCS.

In section $\nabla$, we start by briefly discussing the radiative corrections to elastic electronproton scattering. We apply the radiative corrections to elastic scattering data on the proton. We next give our results for the $e p \rightarrow e p \gamma$ reaction, and indicate how the observables are modified due to the first order QED radiative corrections. We discuss first the polarizability region for the $e p \rightarrow e p \gamma$ reaction, corresponding with a low outgoing photon energy. We show results for both unpolarized and polarized cross sections in MAMI and JLab kinematics. Subsequently, we give the effect of the first order QED radiative corrections to the DVCS cross section and the electron single spin asymmetry.

Finally, we give our conclusions in section $\mathrm{VI}$.

We present technical details needed in the calculations, in two appendices. In appendix $\mathrm{A}$, we calculate the radiative corrections to elastic lepton-nucleon scattering, which serves as a point of comparison with the $e p \rightarrow e p \gamma$ reaction. In particular, we present the details of the calculation of the soft photon emission contributions, and perform analytically the phase space integral over the soft photon in an exact way. We compare with other calculations in the literature. In appendix B, we present some technical details on the integration method used to evaluate singular Feynman parameter integrals. 


\section{LOWEST ORDER AMPLITUDES OF THE $E P \rightarrow E P \gamma$ REACTION}

The lowest order (in $\alpha_{e m}$ ), contributions to the $e p \rightarrow e p \gamma$ reaction are given by the one-photon exchange processes. We denote in this work the four-momenta of the initial and final electrons by $k\left(E_{e}, \vec{k}_{e}\right)$ and $k^{\prime}\left(E_{e}^{\prime}, \vec{k}_{e}^{\prime}\right)$; the four-momenta of the initial and final protons by $p\left(E_{N}, \vec{p}_{N}\right)$ and $p^{\prime}\left(E_{N}^{\prime}, \vec{p}_{N}^{\prime}\right)$; and the four-momentum of the outgoing photon by $q^{\prime}\left(\left|\vec{q}^{\prime}\right|, \vec{q}^{\prime}\right)$. Furthermore, we denote $q \equiv k-k^{\prime}=p^{\prime}-p+q^{\prime}$ and $Q^{2}=-q^{2}>0$. The masses of the electron and proton are denoted by $m$ and $M_{N}$ respectively. The helicities of the initial (final) electrons are denoted by $h\left(h^{\prime}\right)$; the spins of initial (final) protons by $s_{p}\left(s_{p}^{\prime}\right)$; and the polarization four-vector of the outgoing photon by $\varepsilon$. The spinors of initial and final electrons are denoted by $u(k, h)$ and $u\left(k^{\prime}, h^{\prime}\right)$; whereas the spinors of initial and final protons are denoted by $N\left(p, s_{p}\right)$ and $N\left(p^{\prime}, s_{p}^{\prime}\right)$. Throughout this work, we follow the conventions of Bjorken and Drell [25].

In Figs. 1 (BHi) and (BHf), which are known as the Bethe-Heitler (BH) diagrams, a photon is emitted by either the incident or final electrons. The expressions for Figs. 1 (BHi) and (BHf) are respectively given by :

$$
\begin{aligned}
& M_{B H}^{i}=i e^{3} \bar{u}\left(k^{\prime}, h^{\prime}\right) \gamma^{\nu} \frac{\left(\not k-\not q^{\prime}+m\right)}{-2 k \cdot q^{\prime}} k^{*} u(k, h) \frac{1}{\left(p^{\prime}-p\right)^{2}} \bar{N}\left(p^{\prime}, s_{p}^{\prime}\right) \Gamma_{\nu}\left(p^{\prime}, p\right) N\left(p, s_{p}\right), \\
& M_{B H}^{f}=i e^{3} \bar{u}\left(k^{\prime}, h^{\prime}\right) k^{*} \frac{\left(\not k^{\prime}+\not q^{\prime}+m\right)}{2 k^{\prime} \cdot q^{\prime}} \gamma^{\nu} u(k, h) \frac{1}{\left(p^{\prime}-p\right)^{2}} \bar{N}\left(p^{\prime}, s_{p}^{\prime}\right) \Gamma_{\nu}\left(p^{\prime}, p\right) N\left(p, s_{p}\right),
\end{aligned}
$$

where the electron charge is given by $(-e)$ (i.e. $e>0$ in this work). The on-shell electromagnetic vertex at the hadron side $\Gamma_{\nu}$ in Eqs. (1, 2) is given by

$$
\Gamma_{\nu}\left(p^{\prime}, p\right)=F_{1}\left(\left(p^{\prime}-p\right)^{2}\right) \gamma_{\nu}+F_{2}\left(\left(p^{\prime}-p\right)^{2}\right) i \sigma_{\nu \lambda} \frac{\left(p^{\prime}-p\right)^{\lambda}}{2 M_{N}}
$$

where $F_{1}$ and $F_{2}$ are respectively the Dirac and Pauli electromagnetic (on-shell) form factors of the nucleon. The four-momentum squared of the virtual photon in the BH processes is $t=\left(p^{\prime}-p\right)^{2}$, in contrast to $q^{2}$, which is the four-momentum squared for the VCS process $\gamma^{*} p \rightarrow \gamma p$, where the final photon is emitted from the hadron side. This latter part contains the nucleon structure information.

The amplitude of the VCS contribution to the $e^{-} p \rightarrow e^{-} p \gamma$ reaction is given by :

$$
M_{V C S}=-i e^{3} \bar{u}\left(k^{\prime}, h^{\prime}\right) \gamma_{\nu} u(k, h) \frac{1}{q^{2}} \varepsilon_{\mu}^{*} H^{\mu \nu} .
$$

Remark that for a positive lepton, the VCS amplitude changes sign. In Eq. (4), the gaugeinvariant, hadronic tensor $H^{\mu \nu}$ is defined by :

$$
H^{\mu \nu}=-i \int d^{4} x \mathrm{e}^{-i q \cdot x}<p^{\prime}\left|T\left[j^{\nu}(x), j^{\mu}(0)\right]\right| p>
$$

where $T$ represents the time ordering, and $j^{\nu}$ the electromagnetic current operator.

For the DVCS process in the Bjorken limit, the hadronic tensor of Eq. (5) is parametrized in terms of six leading twist skewed parton distributions (see e.g. [16]). 
For the VCS process at low energy, as investigated experimentally in [11 13], an important contribution to the tensor of Eq.(5) originates from the nucleon pole contributions shown in Figs. 1 $(\mathrm{BORNi})$ and $(\mathrm{BORNf})$. The contributions of the Born diagrams to the hadronic tensor are given by :

$$
\begin{aligned}
& H_{B O R N, i}^{\mu \nu}=\bar{N}\left(p^{\prime}, s_{p}^{\prime}\right) \Gamma^{\nu}\left(p^{\prime}, p-q^{\prime}\right) \frac{\left(\not p-\not q^{\prime}+M_{N}\right)}{-2 p \cdot q^{\prime}} \Gamma^{\mu}\left(p-q^{\prime}, p\right) N\left(p, s_{p}\right), \\
& H_{B O R N, f}^{\mu \nu}=\bar{N}\left(p^{\prime}, s_{p}^{\prime}\right) \Gamma^{\mu}\left(p^{\prime}, p^{\prime}+q^{\prime}\right) \frac{\left(\not p^{\prime}+\not q^{\prime}+M_{N}\right)}{2 p^{\prime} \cdot q^{\prime}} \Gamma^{\nu}\left(p^{\prime}+q^{\prime}, p\right) N\left(p, s_{p}\right),
\end{aligned}
$$

where the vertex $\Gamma^{\mu}$ is now evaluated for off mass-shell values of one of its arguments. In Ref. [3], the Born diagrams were evaluated by using the vertex of Eq. (3). Doing so, the Born diagrams are separately gauge invariant. All nucleon structure effects are then absorbed in a non-Born amplitude which is regular in $q^{\prime}$ and for which the Low Energy Theorem (LET) tells that it starts at order $q^{\prime}$. The nucleon structure effects to the VCS tensor (Eq. (5)) below pion threshold, are then parametrized at order $q^{\prime}$ in terms of six generalized (i.e. $Q^{2}$ dependent) nucleon polarizabilities [3, 团.

\section{FIRST ORDER RADIATIVE CORRECTIONS TO THE $E P \rightarrow E P \gamma$ REACTION}

\section{A. Virtual radiative corrections}

In this section, we calculate the one-loop QED virtual radiative corrections to the $e p \rightarrow$ ep $\gamma$ reaction, which are represented in Fig. 2. In the present section, we consider only the corrections originating from the electron side as they can be calculated model-independently. The corrections originating from the hadronic side, for which a nucleon structure model is needed, will be discussed and estimated in section IIID.

The virtual radiative corrections to the $\mathrm{BH}$ process contain vertex corrections : Figs. 2 (V1i - V3i) and (V1f - V3f); electron self-energy corrections : Figs. 2 (Si, Sf); and vacuum polarization corrections : Figs. 2 (P1i, P1f). We indicate in our notation of the different diagrams whether the photon in the $e p \rightarrow e p \gamma$ reaction is emitted from the initial (i) electron or from the final (f) electron.

The part of the virtual radiative corrections to the VCS process (i.e. where the photon in the reaction $e p \rightarrow e p \gamma$ is emitted from the hadronic side) which can be calculated model-independently, consists of the vertex diagram shown in Fig. 2 (V4) and the vacuum polarization diagram shown in Fig. 2 (P2). The blob in those figures represents the VCS process. For VCS below pion threshold, the blob is given by the Born diagrams (Fig. 1 $(\mathrm{BORNi})$ and $(\mathrm{BORNf}))+$ non-Born diagrams, which describe the nucleon polarizability effects. For DVCS, the blob is given in leading order by the so-called handbag diagrams, where the photon hits a quark in the proton [14,15,2].

The calculation of the virtual radiative corrections to the VCS process is similar to that for electron scattering. The virtual radiative corrections to the Bethe-Heitler process are different, but involve the same one-loop building blocks, i.e. electron vertex, electron self-energy and photon self-energy. Therefore, we give in appendix A (sections A1] - A4) 
the derivation and the expressions for these basic building blocks, and we apply it to elastic electron-nucleon scattering. In our calculations, we use the dimensional regularization method to treat both ultraviolet (UV) and infrared (IR) divergences. This amounts to evaluate all loop integrals in $D$ dimensions. The divergences then show up (when one takes $D \rightarrow 4$ ) as poles of the form $1 / \varepsilon$, where $\varepsilon \equiv 2-D / 2$. UV divergences are regularized by taking $D<4$ (i.e. $\varepsilon_{U V}=2-D / 2>0$ ), whereas IR divergences are regularized by taking $D>4$ (i.e. $\varepsilon_{I R}=2-D / 2<0$ ). Care has to be taken as to isolate the UV and IR divergent parts in the loop integrals first, as two different limits are understood when one takes $D=4$ at the end. The technical details of our calculational method can also be found in appendix A. We apply it here to calculate the diagrams of Fig. 2 to the $e p \rightarrow e p \gamma$ reaction.

\section{Vertex correction diagrams of Figs. 国(V1i) and (V1f)}

The amplitude corresponding to Fig. 2 (V1i) is given by

$$
\begin{aligned}
& M_{V 1}^{i}=\frac{e^{5}}{\left(p^{\prime}-p\right)^{2}} \bar{N}\left(p^{\prime}, s_{p}^{\prime}\right) \Gamma_{\nu}\left(p^{\prime}, p\right) N\left(p, s_{p}\right) \\
& \times \bar{u}\left(k^{\prime}, h^{\prime}\right) \gamma^{\nu} \frac{\left(\not k-\not q^{\prime}+m\right)}{-2 k \cdot q^{\prime}} \mu^{4-D} \int \frac{d^{D} l}{(2 \pi)^{D}} \frac{\gamma^{\alpha}\left(\not k-\not q^{\prime}-\not l+m\right) \not^{*}(\not k-\not l+m) \gamma_{\alpha}}{\left[l^{2}\right]\left[l^{2}-2 l . k\right]\left[l^{2}-2 l .\left(k-q^{\prime}\right)-2 k \cdot q^{\prime}\right]} u(k, h),
\end{aligned}
$$

where a mass scale $\mu$ (renormalization scale) is introduced when passing to $D \neq 4$ dimensions in order to keep the coupling constant dimensionless. One sees by inspection that the loop integral in Eq. (8), when taking $D=4$, is IR finite ( $l \rightarrow 0$ behavior), but has an UV divergence ( $l \rightarrow \infty$ behavior). Our strategy to evaluate a complicated loop integral as in Eq. (8), is to solve first a simpler loop integral which contains entirely the UV divergence and which can be done analytically more easily. We observe from Eq. (8) that only the term

in the numerator proportional to $\gamma^{\alpha} \Lambda \varepsilon^{*} \lambda \gamma_{\alpha}$ is responsible for the UV divergence. To evaluate it, we add a similar term by replacing one factor in the denominator and evaluate this term analytically. In order to obtain the equivalence with $M_{v 1}^{i}$, we have to subtract the added term again from the expression of Eq. (8). This leads to

$$
\begin{aligned}
& M_{V 1}^{i}=\frac{e^{5}}{\left(p^{\prime}-p\right)^{2}} \bar{N}\left(p^{\prime}, s_{p}^{\prime}\right) \Gamma_{\nu}\left(p^{\prime}, p\right) N\left(p, s_{p}\right) \\
& \times \bar{u}\left(k^{\prime}, h^{\prime}\right) \gamma^{\nu} \frac{\left(\not k-\not q^{\prime}+m\right)}{-2 k \cdot q^{\prime}}\left\{\mu^{4-D} \int \frac{d^{D} l}{(2 \pi)^{D}} \frac{\gamma^{\alpha} \not \not^{*} \not \gamma_{\alpha}}{\left[l^{2}\right]\left[l^{2}-2 l . k\right]\left[l^{2}-2 l . k^{\prime}\right]}\right. \\
& +2 \int \frac{d^{4} l}{(2 \pi)^{4}} \frac{\not \not k^{*}\left(\not k-\not q^{\prime}\right)+\not k \not k^{*} \not l-m^{2} k^{*}+4 m \varepsilon^{*} .(k-l)-\not k \not k^{*}\left(\not k-\not q^{\prime}\right)}{\left[l^{2}\right]\left[l^{2}-2 l . k\right]\left[l^{2}-2 l .\left(k-q^{\prime}\right)-2 k \cdot q^{\prime}\right]} \\
& \left.+2 \int \frac{d^{4} l}{(2 \pi)^{4}} \frac{-2 \not k^{*} \not l\left[l .\left(q-q^{\prime}\right)+k \cdot q^{\prime}\right]}{\left[l^{2}\right]\left[l^{2}-2 l . k\right]\left[l^{2}-2 l . k^{\prime}\right]\left[l^{2}-2 l .\left(k-q^{\prime}\right)-2 k \cdot q^{\prime}\right]}\right\} u(k, h) \text {. }
\end{aligned}
$$

It should be remarked that only the added term in Eq. (9) (first term within curly brackets of Eq. (9) ) is UV divergent and has therefore to be evaluated in $D$ dimensions using the dimensional regularization method. The third term within the curly brackets in Eq. (9) is 
the difference between the term proportional to $\gamma^{\alpha} \not k^{*} \not \gamma_{\alpha}$ in Eq. (8) and the added term (which has one different factor in the denominator). As can be seen by power counting, this term is UV finite and can therefore readily be evaluated for $D=4$. The denominator in the UV divergent first term of Eq. (9), was chosen so that it corresponds with the vertex correction which appears in electron scattering. Therefore, this UV divergent term can be calculated analytically along a similar way as was performed in appendix A. The result is given by :

$$
\begin{aligned}
& \mu^{4-D} \int \frac{d^{D} l}{(2 \pi)^{D}} \frac{\gamma^{\alpha} \not k^{*} \not \gamma_{\alpha}}{\left[l^{2}\right]\left[l^{2}-2 l . k\right]\left[l^{2}-2 l . k^{\prime}\right]} \\
& =\frac{i}{(4 \pi)^{2}}\left\{k^{*}\left[\frac{1}{\varepsilon_{U V}}-\gamma_{E}+\ln \left(\frac{4 \pi \mu^{2}}{m^{2}}\right)+1-v \ln \left(\frac{v+1}{v-1}\right)\right]-\frac{1}{Q^{2}} \not q k^{*} \not q\right. \\
& \left.\quad+\frac{1}{Q^{2} v} \ln \left(\frac{v+1}{v-1}\right)\left[\not k k^{*} \not k^{\prime}+\not k^{\prime} \not k^{*} \not k+\left(\frac{v^{2}+1}{2}\right) \not q \not k^{*} \not q\right]\right\},
\end{aligned}
$$

where $v$ is defined as

$$
v^{2} \equiv 1+\frac{4 m^{2}}{Q^{2}}
$$

The UV divergence in Eq. (10) is removed by the corresponding vertex counterterm as given by Eqs. (A6, A16)

$$
(C T)_{V 1}^{i}=M_{B H}^{i} \frac{\left(-e^{2}\right)}{(4 \pi)^{2}}\left\{\left[\frac{1}{\varepsilon_{U V}}-\gamma_{E}+\ln \left(\frac{4 \pi \mu^{2}}{m^{2}}\right)\right]+2\left[\frac{1}{\varepsilon_{I R}}-\gamma_{E}+\ln \left(\frac{4 \pi \mu^{2}}{m^{2}}\right)\right]+4\right\},
$$

where we have used the expression of Eq. (1) for the BH amplitude $M_{B H}^{i}$. Adding the counterterm of Eq. (12) to Eq. (9) and introducing a Feynman parametrization in the second and third terms of Eq. (9) in order to perform the integrals over $l$, yields the total, UV finite result :

$$
\begin{aligned}
& M_{V 1}^{i}+(C T)_{V 1}^{i}=M_{B H}^{i} \frac{e^{2}}{(4 \pi)^{2}}\left\{-2\left[\frac{1}{\varepsilon_{I R}}-\gamma_{E}+\ln \left(\frac{4 \pi \mu^{2}}{m^{2}}\right)\right]-3-v \ln \left(\frac{v+1}{v-1}\right)\right\} \\
& +\frac{i e^{5}}{(4 \pi)^{2}} \frac{1}{\left(p^{\prime}-p\right)^{2}} \bar{N}\left(p^{\prime}, s_{p}^{\prime}\right) \Gamma_{\nu}\left(p^{\prime}, p\right) N\left(p, s_{p}\right) \\
& \times \bar{u}\left(k^{\prime}, h^{\prime}\right) \gamma^{\nu} \frac{\left(\not k-\not q^{\prime}+m\right)}{-2 k \cdot q^{\prime}} \\
& \times\left\{\frac{1}{Q^{2}}\left[\left(-1+\frac{v^{2}+1}{2 v} \ln \left(\frac{v+1}{v-1}\right)\right) \not q k^{*} \not q+\frac{1}{v} \ln \left(\frac{v+1}{v-1}\right)\left\{\not k \not k^{*} \not k^{\prime}+\not k^{\prime} \not k^{*} \not k\right\}\right]\right. \\
& -2 \int_{0}^{1} d y \int_{0}^{1} d x \frac{1}{B_{1}^{i}}\left[y\left(\not k-\not q^{\prime} x\right) k^{*}\left(\not k-\not q^{\prime}\right)+y \not k \not k^{*}\left(\not k-\not q^{\prime} x\right)\right. \\
& \left.\quad+4 m\left(\varepsilon^{*} . k\right)(1-y)-\not k \not k^{*}\left(\not k-\not q^{\prime}\right)-m^{2} \not k^{*}\right]
\end{aligned}
$$




$$
\begin{aligned}
-4 \int_{0}^{1} d x_{3} x_{3}^{2} \int_{0}^{1} d x_{2} x_{2} \int_{0}^{1} d x_{1} & {\left[\left(\frac{1}{A^{i}} \not k^{*}+\frac{1}{\left(A^{i}\right)^{2}} P^{i} \not^{*} P^{i}\right)\left(P^{i} \cdot\left(q-q^{\prime}\right)+k \cdot q^{\prime}\right)\right.} \\
& \left.\left.-\frac{1}{2 A^{i}}\left(\left(\not q-\not q^{\prime}\right) \xi^{*} P^{i}+P^{i} \xi^{*}\left(\not q-\not q^{\prime}\right)\right)\right]\right\} u(k, h),
\end{aligned}
$$

with the four-vector $P^{i}$ defined by

$$
P^{i} \equiv\left(k-q^{\prime}\right)\left(1-x_{3}\right)+\left(k-q x_{1}\right) x_{2} x_{3},
$$

and the scalars $A^{i}$ and $B^{i}$ defined by

$$
A^{i} \equiv 2 k \cdot q^{\prime}\left(1-x_{3}\right)+\left(P^{i}\right)^{2}
$$

and

$$
B_{1}^{i} \equiv 2 k \cdot q^{\prime} x(1-y)+m^{2} y .
$$

Remark that although Eq. (13) is UV finite, it contains now an IR divergence through the vertex counterterm of Eq. (12) as shown in appendix A (Eq. (A16)). We will demonstrate however in section [IIB, that all IR divergences, arising from the one-loop corrections to the $e p \rightarrow e p \gamma$ reaction, are cancelled when adding the corresponding soft photon emission contributions.

The Feynman parameter integrals in Eq. (13) which orginate from the finite integrals in Eq. (9) remain to be evaluated. As an analytical calculation of these integrals is rather complicated, we will evaluate them numerically in this paper, which will be discussed in section $\amalg$ IIC.

In a completely similar way as for Fig. 2 (V1i), the total amplitude including the counterterm corresponding to Fig. 2 (V1f) yields :

$$
\begin{aligned}
& M_{V 1}^{f}+(C T)_{V 1}^{f}=M_{B H}^{f} \frac{e^{2}}{(4 \pi)^{2}}\left\{-2\left[\frac{1}{\varepsilon_{I R}}-\gamma_{E}+\ln \left(\frac{4 \pi \mu^{2}}{m^{2}}\right)\right]-3-v \ln \left(\frac{v+1}{v-1}\right)\right\} \\
& +\frac{i e^{5}}{(4 \pi)^{2}} \frac{1}{\left(p^{\prime}-p\right)^{2}} \bar{N}\left(p^{\prime}, s_{p}^{\prime}\right) \Gamma_{\nu}\left(p^{\prime}, p\right) N\left(p, s_{p}\right) \\
& \times \bar{u}\left(k^{\prime}, h^{\prime}\right)\left\{\frac{1}{Q^{2}}\left[\left(-1+\frac{v^{2}+1}{2 v} \ln \left(\frac{v+1}{v-1}\right)\right) \not q k^{*} \not q+\frac{1}{v} \ln \left(\frac{v+1}{v-1}\right)\left\{\not k \not k^{*} \not k^{\prime}+\not k^{\prime} k^{*} \not k\right\}\right]\right. \\
& -2 \int_{0}^{1} d y \int_{0}^{1} d x \frac{1}{B_{1}^{f}}\left[y\left(\not k^{\prime}+\not q^{\prime}\right) \not k^{*}\left(\not k^{\prime}+\not q^{\prime} x\right)+y\left(\not k^{\prime}+\not q^{\prime} x\right) \not k^{*} \not k^{\prime}\right. \\
& +4 \int_{0}^{1} d x_{3} x_{3}^{2} \int_{0}^{1} d x_{2} x_{2} \int_{0}^{1} d x_{1}\left[\left(\frac{1}{A^{f}} \not k^{*}+\frac{1}{\left(A^{f}\right)^{2}} \not P^{f} \not k^{*} \not P^{f}\right)\left(P^{f} \cdot\left(q-q^{\prime}\right)+k^{\prime} \cdot q^{\prime}\right)\right. \\
& \left.\quad \frac{\left(\not k^{\prime}+\not q^{\prime}+m\right)}{2 k^{\prime} \cdot q^{\prime}} \gamma^{\nu} u(k, h), k^{\prime}\right]
\end{aligned}
$$


with the four-vector $P^{f}$ defined by

$$
P^{f} \equiv\left(k^{\prime}+q^{\prime}\right)\left(1-x_{3}\right)+\left(k^{\prime}+q x_{1}\right) x_{2} x_{3},
$$

and the scalars $A^{f}$ and $B_{1}^{f}$ defined by

$$
A^{f} \equiv-2 k^{\prime} \cdot q^{\prime}\left(1-x_{3}\right)+\left(P^{f}\right)^{2}
$$

and

$$
B_{1}^{f} \equiv-2 k^{\prime} \cdot q^{\prime} x(1-y)+m^{2} y
$$

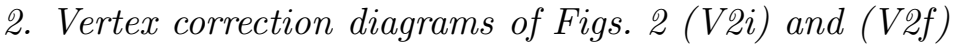

The amplitude corresponding to Fig. 2 (V2i) is given by

$$
\begin{aligned}
& M_{V 2}^{i}=\frac{e^{5}}{\left(p^{\prime}-p\right)^{2}} \bar{N}\left(p^{\prime}, s_{p}^{\prime}\right) \Gamma_{\nu}\left(p^{\prime}, p\right) N\left(p, s_{p}\right) \\
& \times \bar{u}\left(k^{\prime}, h^{\prime}\right) \mu^{4-D} \int \frac{d^{D} l}{(2 \pi)^{D}} \frac{\left.\gamma^{\alpha}\left(\not k^{\prime}-\not l+m\right) \gamma^{\nu}\left(\not k-\not q^{\prime}-\not l+m\right) \gamma_{\alpha}\right]\left[\left(k-\not l^{2}-2 l . k^{\prime}\right]\left[l^{2}-2 l .\left(k-q^{\prime}\right)-2 k \cdot q^{\prime}\right]\right.}{-2 k \cdot q^{\prime}} k^{*} u(k, h) .
\end{aligned}
$$

One sees from Eq. (21) that again only the term in the numerator proportional to $\gamma^{\alpha} \not \gamma^{\nu} \not \gamma_{\alpha}$ contains an UV divergence for $D=4$. To evaluate the loop integral of Eq. (21), we therefore apply a similar trick as used before in Eq. (9). This amounts to adding and subtracting a term in Eq. (21) by replacing $\left(l^{2}-2 l .\left(k-q^{\prime}\right)-2 k \cdot q^{\prime}\right)$ in the denominator by $\left(l^{2}-2 l . k^{\prime}\right)$, and which contains entirely the UV divergence. The further steps are then analogous to those following Eq. (9), and yield the following result for Fig. 2 (V2i) :

$$
\begin{aligned}
& M_{V 2}^{i}+(C T)_{V 2}^{i}=M_{B H}^{i} \frac{e^{2}}{(4 \pi)^{2}}\left\{-2\left[\frac{1}{\varepsilon_{I R}}-\gamma_{E}+\ln \left(\frac{4 \pi \mu^{2}}{m^{2}}\right)\right]-3-v \ln \left(\frac{v+1}{v-1}\right)\right\} \\
& +\frac{i e^{5}}{(4 \pi)^{2}} \frac{1}{\left(p^{\prime}-p\right)^{2}} \bar{N}\left(p^{\prime}, s_{p}^{\prime}\right) \Gamma_{\nu}\left(p^{\prime}, p\right) N\left(p, s_{p}\right) \\
& \times \bar{u}\left(k^{\prime}, h^{\prime}\right)\left\{\frac{1}{Q^{2}}\left[\left(-1+\frac{v^{2}+1}{2 v} \ln \left(\frac{v+1}{v-1}\right)\right) \not \gamma^{\nu} \not q+\frac{1}{v} \ln \left(\frac{v+1}{v-1}\right)\left\{\not k \gamma^{\nu} \not k^{\prime}+\not k^{\prime} \gamma^{\nu} \not k\right\}\right\}\right. \\
& -2 \int_{0}^{1} d y \int_{0}^{1} d x \frac{1}{B_{2}^{i}}\left[y\left(\not k-\not q^{\prime}\right) \gamma^{\nu}\left(\not k^{\prime}+\left(\not q-\not q^{\prime}\right) x\right)+y\left(\not k^{\prime}+\left(\not q-\not q^{\prime}\right) x\right) \gamma^{\nu} \not k^{\prime}\right. \\
& -4 \int_{0}^{1} d x_{3} x_{3}^{2} \int_{0}^{1} d x_{2} x_{2} \int_{0}^{1} d x_{1}\left[\left(\frac{1}{A^{i}} \gamma^{\nu}+\frac{1}{\left(A^{i}\right)^{2}} \not P^{i} \gamma^{\nu} \not P^{i}\right)\left(q^{\prime} \cdot\left(k-P^{i}\right)\right)\right. \\
& \} \frac{\left(\not k-\not k^{\prime}+m\right)}{-2 k \cdot q^{\prime}} k^{*} u(k, h), m^{\prime} \gamma^{\nu}\right]
\end{aligned}
$$


where $A^{i}$ is given as in Eq. (15) and where

$$
B_{2}^{i} \equiv m^{2} y+x^{2} y\left(q-q^{\prime}\right)^{2}+2 x k \cdot q^{\prime}+2 x y k^{\prime} \cdot\left(q-q^{\prime}\right)
$$

In an analogous way, the amplitude corresponding to Fig. 22 (V2f) can be calculated, and yields as result :

$$
\begin{aligned}
& M_{V 2}^{f}+(C T)_{V 2}^{f}=M_{B H}^{f} \frac{e^{2}}{(4 \pi)^{2}}\left\{-2\left[\frac{1}{\varepsilon_{I R}}-\gamma_{E}+\ln \left(\frac{4 \pi \mu^{2}}{m^{2}}\right)\right]-3-v \ln \left(\frac{v+1}{v-1}\right)\right\} \\
& +\frac{i e^{5}}{(4 \pi)^{2}} \frac{1}{\left(p^{\prime}-p\right)^{2}} \bar{N}\left(p^{\prime}, s_{p}^{\prime}\right) \Gamma_{\nu}\left(p^{\prime}, p\right) N\left(p, s_{p}\right) \\
& \times \bar{u}\left(k^{\prime}, h^{\prime}\right) \not k^{*} \frac{\left(\not k^{\prime}+\not q^{\prime}+m\right)}{2 k^{\prime} \cdot q^{\prime}} \\
& \times\left\{\frac{1}{Q^{2}}\left[\left(-1+\frac{v^{2}+1}{2 v} \ln \left(\frac{v+1}{v-1}\right)\right) \not q \gamma^{\nu} \not q+\frac{1}{v} \ln \left(\frac{v+1}{v-1}\right)\left\{\not k \gamma^{\nu} \not k^{\prime}+\not k^{\prime} \gamma^{\nu} \not k\right\}\right\}\right. \\
& -2 \int_{0}^{1} d y \int_{0}^{1} d x \frac{1}{B_{2}^{i}}\left[y \not k \gamma^{\nu}\left(\not k-\left(\not q-\not q^{\prime}\right) x\right)+y\left(\not k-\left(\not q-\not q^{\prime}\right) x\right) \gamma^{\nu}\left(\not k^{\prime}+\not q^{\prime}\right)\right. \\
& +4 \int_{0}^{1} d x_{3} x_{3}^{2} \int_{0}^{1} d x_{2} x_{2} \int_{0}^{1} d x_{1}\left[\left(\frac{1}{A^{f}} \gamma^{\nu}+\frac{1}{\left(A^{f}\right)^{2}} \not P^{f} \gamma^{\nu} \not P^{f}\right)\left(q^{\prime} \cdot\left(k^{\prime}-P^{f}\right)\right)\right. \\
& \left.+4 m(k)^{\nu}(1-y)-\not k \gamma^{\nu}\left(\not k^{\prime}+\not q^{\prime}\right)-m^{2} \gamma^{\nu}\right]
\end{aligned}
$$

where $A^{f}$ is given as in Eq. (19) and where

$$
B_{2}^{f} \equiv m^{2} y+x^{2} y\left(q-q^{\prime}\right)^{2}-2 x k^{\prime} \cdot q^{\prime}-2 x y k \cdot\left(q-q^{\prime}\right)
$$

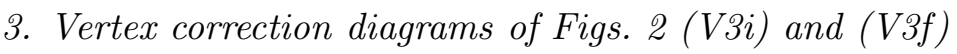

The amplitude $M_{V 3}^{i}$ corresponding to Fig. 2 (V3i) is given by

$$
\begin{aligned}
& M_{V 3}^{i}=\frac{e^{5}}{\left(p^{\prime}-p\right)^{2}} \bar{N}\left(p^{\prime}, s_{p}^{\prime}\right) \Gamma_{\nu}\left(p^{\prime}, p\right) N\left(p, s_{p}\right) \\
& \times \bar{u}\left(k^{\prime}, h^{\prime}\right) \mu^{4-D} \int \frac{d^{D} l}{(2 \pi)^{D}} \frac{\gamma^{\alpha}\left(\not k^{\prime}+\not l+m\right) \gamma^{\nu}\left(\not k-\not q^{\prime}+\not l+m\right) k^{*}(\not k+\not l+m) \gamma_{\alpha}}{\left[l^{2}\right]\left[l^{2}+2 l . k^{\prime}\right]\left[l^{2}+2 l . k\right]\left[l^{2}+2 l .\left(k-q^{\prime}\right)-2 k . q^{\prime}\right]} u(k, h) .
\end{aligned}
$$

Remark that the loop integral in Eq. (26) is UV finite but contains an IR divergence for $D=4$. This is because in Fig. 2 (V3i), a soft virtual photon $(l \rightarrow 0)$ couples to two on-shell electron lines. To isolate the IR divergence, we first decompose the 
numerator in Eq. (26) by using the relations $\bar{u}\left(k^{\prime}, h^{\prime}\right) \gamma^{\alpha}\left(k^{\prime}+m\right)=\bar{u}\left(k^{\prime}, h^{\prime}\right) 2 k_{\alpha}^{\prime}$ and $(k+m) \gamma_{\alpha} u(k, h)=2 k_{\alpha} u(k, h)$. This yields :

$$
\begin{aligned}
& M_{V 3}^{i}=\frac{e^{5}}{\left(p^{\prime}-p\right)^{2}} \bar{N}\left(p^{\prime}, s_{p}^{\prime}\right) \Gamma_{\nu}\left(p^{\prime}, p\right) N\left(p, s_{p}\right) \\
& \times \bar{u}\left(k^{\prime}, h^{\prime}\right) \mu^{4-D} \int \frac{d^{D} l}{(2 \pi)^{D}} \frac{1}{\left[l^{2}\right]\left[l^{2}+2 l \cdot k^{\prime}\right]\left[l^{2}+2 l . k\right]\left[l^{2}+2 l .\left(k-q^{\prime}\right)-2 k \cdot q^{\prime}\right]} \\
& \times\left\{4\left(k . k^{\prime}\right) \gamma^{\nu}\left(\not k-\not q^{\prime}+m\right) k^{*}+4\left(k \cdot k^{\prime}\right) \gamma^{\nu} \not \not k^{*}+2 \gamma^{\nu}\left(\not k-\not q^{\prime}+\not \lambda+m\right) k^{*} \not \not^{\prime}\right. \\
& \left.+2 \not k \not \gamma^{\nu}\left(\not k-\not q^{\prime}+\not l+m\right) k^{*}+\gamma^{\alpha} \not \gamma \gamma^{\nu}\left(\not k-\not q^{\prime}+\not l+m\right) \not k^{*} \not \gamma_{\alpha}\right\} u(k, h) .
\end{aligned}
$$

In Eq. (27), only the term in the numerator which is $l$-independent (the first term within the curly brackets) contains an IR divergence, whereas all the other terms are finite. As before, instead of aiming at an analytical formula for a rather complicated integral, we evaluate the IR divergent part of the integral in Eq. (27) by adding and subtracting a term that contains the divergence and that can be performed analytically rather easily. In constructing this term, we are looking for a denominator which contains the same dependence as the basic $\mathrm{BH}$ process in order that this $\mathrm{BH}$ amplitude can be factored from this IR divergent term. This yields the following expression, which is by construction identical to Eq. (27) :

$$
\begin{aligned}
& M_{V 3}^{i}=\frac{e^{5}}{\left(p^{\prime}-p\right)^{2}} \bar{N}\left(p^{\prime}, s_{p}^{\prime}\right) \Gamma_{\nu}\left(p^{\prime}, p\right) N\left(p, s_{p}\right) \\
& \times \bar{u}\left(k^{\prime}, h^{\prime}\right)\left\{\mu^{4-D} \int \frac{d^{D} l}{(2 \pi)^{D}} \frac{4\left(k . k^{\prime}\right) \gamma^{\nu}\left(\not k-\not q^{\prime}+m\right) k^{*}}{\left[l^{2}\right]\left[l^{2}+2 l . k^{\prime}\right]\left[l^{2}+2 l . k\right]\left[-2 k . q^{\prime}\right]}\right. \\
& +\int \frac{d^{4} l}{(2 \pi)^{4}} \frac{1}{\left[l^{2}\right]\left[l^{2}+2 l . k^{\prime}\right]\left[l^{2}+2 l . k\right]\left[l^{2}+2 l .\left(k-q^{\prime}\right)-2 k . q^{\prime}\right]} \\
& \times\left[4\left(k . k^{\prime}\right) \gamma^{\nu}\left(\not k-\not q^{\prime}+m\right) k^{*} \frac{-l^{2}-2 l .\left(k-q^{\prime}\right)}{-2 k \cdot q^{\prime}}+4\left(k . k^{\prime}\right) \gamma^{\nu} \not \xi^{*}\right. \\
& +2 \gamma^{\nu}\left(\not k-\not q^{\prime}+\not 1+m\right) k^{*} \not \not^{\prime}+2 \not k \not \gamma^{\nu}\left(\not k-\not q^{\prime}+\not \lambda+m\right) k^{*} \\
& \left.\left.+\gamma^{\alpha} \not \gamma^{\nu}\left(\not k-\not q^{\prime}+\not l+m\right) k^{*} \not \gamma_{\alpha}\right]\right\} u(k, h) \text {. }
\end{aligned}
$$

Remark that the added term (first term of Eq. (28)) contains the IR divergence whereas the other terms of Eq. (28)) do not have any divergences so that the corresponding integrals may be performed directly in four dimensions as indicated. For the first term of Eq. (28) we furthermore see that the $l$-independent part of the energy denominator is the same as the one occuring in the corresponding Bethe-Heitler diagram (Fig. 17a). The l-dependent part of the energy denominator for this term is the same as the one for the vertex correction to elastic electron scattering, Eq. (A9). The corresponding integral may therefore be evaluated analytically in a similar way as was done in appendix A. This yields for the IR divergent term in Eq. (28) :

$$
\frac{e^{5}}{\left(p^{\prime}-p\right)^{2}} \bar{N}\left(p^{\prime}, s_{p}^{\prime}\right) \Gamma_{\nu}\left(p^{\prime}, p\right) N\left(p, s_{p}\right)
$$




$$
\begin{aligned}
& \times \bar{u}\left(k^{\prime}, h^{\prime}\right) \gamma^{\nu} \frac{\left(\not k-\not q^{\prime}+m\right)}{-2 k \cdot q^{\prime}} k^{*} u(k, h) \mu^{4-D} \int \frac{d^{D} l}{(2 \pi)^{D}} \frac{4\left(k . k^{\prime}\right)}{\left[l^{2}\right]\left[l^{2}+2 l . k^{\prime}\right]\left[l^{2}+2 l . k\right]} \\
& =M_{B H}^{i} \frac{e^{2}}{(4 \pi)^{2}}\left\{\left[\frac{1}{\varepsilon_{I R}}-\gamma_{E}+\ln \left(\frac{4 \pi \mu^{2}}{m^{2}}\right)\right] \frac{v^{2}+1}{v} \ln \left(\frac{v+1}{v-1}\right)\right. \\
& \left.\quad+\frac{v^{2}+1}{2 v} \ln \left(\frac{v+1}{v-1}\right) \ln \left(\frac{v^{2}-1}{4 v^{2}}\right)+\frac{v^{2}+1}{v}\left[S p\left(\frac{v+1}{2 v}\right)-S p\left(\frac{v-1}{2 v}\right)\right]\right\} .
\end{aligned}
$$

The evaluation of the finite four-dimensional integral in Eq. (28) can be performed at the expense of the introduction of three Feynman parameter integrals due to the four energy denominators :

$$
\begin{aligned}
& \frac{1}{\left[l^{2}\right]\left[l^{2}+2 l . k^{\prime}\right]\left[l^{2}+2 l . k\right]\left[l^{2}+2 l .\left(k-q^{\prime}\right)-2 k . q^{\prime}\right]} \\
& =6 \int_{0}^{1} d y y^{2} \int_{0}^{1} d x_{2} x_{2} \int_{0}^{1} d x_{1} \frac{1}{\left[\left(l+y P_{x_{1} x_{2}}^{i}\right)^{2}-y C^{i}\right]^{4}},
\end{aligned}
$$

with the four-vector $P_{x_{1} x_{2}}^{i}$ defined by

$$
P_{x_{1} x_{2}}^{i} \equiv\left(q-x_{1} q^{\prime}\right) x_{2}+k^{\prime}
$$

and the scalar $C^{i}$ defined by

$$
C^{i} \equiv 2 k \cdot q^{\prime} x_{1} x_{2}+y\left(P_{x_{1} x_{2}}^{i}\right)^{2} .
$$

The final result for the amplitude $M_{V 3}^{i}$ is then given by

$$
\begin{aligned}
& M_{V 3}^{i}=M_{B H}^{i} \frac{e^{2}}{(4 \pi)^{2}}\left\{\left[\frac{1}{\varepsilon_{I R}}-\gamma_{E}+\ln \left(\frac{4 \pi \mu^{2}}{m^{2}}\right)\right] \frac{v^{2}+1}{v} \ln \left(\frac{v+1}{v-1}\right)\right. \\
& \left.+\frac{v^{2}+1}{2 v} \ln \left(\frac{v+1}{v-1}\right) \ln \left(\frac{v^{2}-1}{4 v^{2}}\right)+\frac{v^{2}+1}{v}\left[S p\left(\frac{v+1}{2 v}\right)-S p\left(\frac{v-1}{2 v}\right)\right]\right\} \\
& +\frac{i e^{5}}{(4 \pi)^{2}} \frac{1}{\left(p^{\prime}-p\right)^{2}} \bar{N}\left(p^{\prime}, s_{p}^{\prime}\right) \Gamma_{\nu}\left(p^{\prime}, p\right) N\left(p, s_{p}\right) \int_{0}^{1} d y y \int_{0}^{1} d x_{2} x_{2} \int_{0}^{1} d x_{1} \\
& \times \bar{u}\left(k^{\prime}, h^{\prime}\right)\left\{\gamma^{\nu} \frac{\left(k-\not q^{\prime}+m\right)}{-2 k \cdot q^{\prime}} k^{*} 4 k \cdot k^{\prime}\left[\frac{2}{C^{i}}+\frac{1}{\left(C^{i}\right)^{2}}\left(-y\left(P_{x_{1} x_{2}}^{i}\right)^{2}+2 P_{x_{1} x_{2}}^{i} \cdot\left(k-q^{\prime}\right)\right)\right]\right. \\
& \frac{2}{C^{i}}\left[\gamma^{\nu} k^{*} \not k^{\prime}+\not k \gamma^{\nu} \xi^{*}-4 m \varepsilon^{* \nu}-\gamma^{\nu}\left(\not k-\not q^{\prime}-y P_{x_{1} x_{2}}^{i}\right) \xi^{*}+y k^{*} \gamma^{\nu} P_{x_{1} x_{2}}^{i}+y P_{x_{1} x_{2}}^{i} k^{*} \gamma^{\nu}\right] \\
& +\frac{1}{\left(C^{i}\right)^{2}}\left[-4\left(k . k^{\prime}\right) \gamma^{\nu} p_{x_{1} x_{2}}^{i} k^{*}-2 \gamma^{\nu}\left(\not k-\not q^{\prime}-y p_{x_{1} x_{2}}^{i}+m\right) k^{*} \mathbb{P}_{x_{1} x_{2}}^{i} \not k^{\prime}\right. \\
& -2 \not k P_{x_{1} x_{2}}^{i} \gamma^{\nu}\left(\not k-\not q^{\prime}-y P_{x_{1} x_{2}}^{i}+m\right) k^{*} \\
& \left.\left.+y P_{x_{1} x_{2}}^{i}\left(-2 k^{*}\left(\not k-\not q^{\prime}-y P_{x_{1} x_{2}}^{i}\right) \gamma^{\nu}+4 m \varepsilon^{* \nu}\right) P_{x_{1} x_{2}}^{i}\right]\right\} u(k, h) .
\end{aligned}
$$


The Feynman parameter integrals in Eq. (33) will be performed numerically as explained in Section IIIC.

In an analogous way, the result for the amplitude $M_{V 3}^{f}$ corresponding to Fig. 2 (V3f) can be calculated, and yields as result :

$$
\begin{aligned}
& M_{V 3}^{f}=M_{B H}^{f} \frac{e^{2}}{(4 \pi)^{2}}\left\{\left[\frac{1}{\varepsilon_{I R}}-\gamma_{E}+\ln \left(\frac{4 \pi \mu^{2}}{m^{2}}\right)\right] \frac{v^{2}+1}{v} \ln \left(\frac{v+1}{v-1}\right)\right. \\
& \left.+\frac{v^{2}+1}{2 v} \ln \left(\frac{v+1}{v-1}\right) \ln \left(\frac{v^{2}-1}{4 v^{2}}\right)+\frac{v^{2}+1}{v}\left[S p\left(\frac{v+1}{2 v}\right)-S p\left(\frac{v-1}{2 v}\right)\right]\right\} \\
& +\frac{i e^{5}}{(4 \pi)^{2}} \frac{1}{\left(p^{\prime}-p\right)^{2}} \bar{N}\left(p^{\prime}, s_{p}^{\prime}\right) \Gamma_{\nu}\left(p^{\prime}, p\right) N\left(p, s_{p}\right) \int_{0}^{1} d y y \int_{0}^{1} d x_{2} x_{2} \int_{0} d x_{1} \\
& \times \bar{u}\left(k^{\prime}, h^{\prime}\right)\left\{k^{*} \frac{\left(\not k^{\prime}+\not q^{\prime}+m\right)}{2 k^{\prime} \cdot q^{\prime}} \gamma^{\nu} 4 k \cdot k^{\prime}\left[\frac{2}{C^{f}}+\frac{1}{\left(C^{f}\right)^{2}}\left(-y\left(P_{x_{1} x_{2}}^{f}\right)^{2}+2 P_{x_{1} x_{2}}^{f} \cdot\left(k^{\prime}+q^{\prime}\right)\right)\right]\right. \\
& +\frac{2}{C f}\left[k^{*} \gamma^{\nu} \not k^{\prime}+\not k k^{*} \gamma^{\nu}-4 m \varepsilon^{* \nu}-k^{*}\left(\not k^{\prime}+\not q^{\prime}-y \not P_{x_{1} x_{2}}^{f}\right) \gamma^{\nu}+y \gamma^{\nu} k^{*} P_{x_{1} x_{2}}^{f}+y \not P_{x_{1} x_{2}}^{f} \gamma^{\nu} k^{*}\right] \\
& +\frac{1}{\left(C^{f}\right)^{2}}\left[-4\left(k \cdot k^{\prime}\right) k^{*} \not P_{x_{1} x_{2}}^{f} \gamma^{\nu}-2 \not k^{*}\left(\not k^{\prime}+\not q^{\prime}-y P_{x_{1} x_{2}}^{f}+m\right) \gamma^{\nu} P_{x_{1} x_{2}}^{f} \not k^{\prime}\right. \\
& -2 \not k \not P_{x_{1} x_{2}}^{f} k^{*}\left(\not k^{\prime}+\not q^{\prime}-y \not P_{x_{1} x_{2}}^{f}+m\right) \gamma^{\nu} \\
& \left.\left.+y \not P_{x_{1} x_{2}}^{f}\left(-2 \gamma^{\nu}\left(\not k^{\prime}+\not q^{\prime}-y \not P_{x_{1} x_{2}}^{f}\right) k^{*}+4 m \varepsilon^{* \nu}\right) \not P_{x_{1} x_{2}}^{f}\right]\right\} u(k, h),
\end{aligned}
$$

with the four-vector $P_{x_{1} x_{2}}^{f}$ defined by

$$
P_{x_{1} x_{2}}^{f} \equiv-\left(q-x_{1} q^{\prime}\right) x_{2}+k
$$

and the scalar $C^{f}$ defined by

$$
C^{f} \equiv-2 k^{\prime} \cdot q^{\prime} x_{1} x_{2}+y\left(P_{x_{1} x_{2}}^{f}\right)^{2}
$$

Remark that in the vertex correction diagrams where the photon couples to the final electron (diagrams of Fig. 2 denoted by $f$ ), the invariant mass of the virtual $\left(e^{-}+\gamma^{*}\right)$ state in the loop is given by $m^{2}+2 k^{\prime} \cdot q^{\prime} \geq m^{2}$. This means that an on-shell propagation is possible for the $\left(e^{-}+\gamma^{*}\right)$ state. This translates mathematically into the presence of integrable singularities in the corresponding Feynman parameter integrals of Eqs. (17, 24) and (34), and yields an imaginary part for the corresponding amplitude. In contrast, in the vertex correction diagrams where the photon couples to the initial electron (diagrams of Fig. 2 denoted by $i)$, the invariant mass of the virtual $\left(e^{-}+\gamma^{*}\right)$ system in the loop is given by $m^{2}$ which means that the corresponding integrals contain no singularities. The numerical treatment of those singular Feynman parameter integrals will be discussed in section $\amalg$ IIC. 
We next evaluate the electron self-energy diagrams of Figs. 2 ( Si) and (Sf). We only have to consider those diagrams where a photon is emitted and re-absorbed by an intermediate electron line. The diagrams with a loop on the initial or final electron lines are already absorbed in the wavefunction and electron mass renormalization, and therefore do not yield an additional correction. This can also be seen from the expression Eq. (A30) for the renormalized lepton self-energy, which vanishes on-shell.

The amplitude corresponding to Fig. 2 ( $\mathrm{Si}$ ) is then given by :

$$
\begin{aligned}
M_{S i} & =i e^{3} \frac{1}{\left(p^{\prime}-p\right)^{2}} \bar{N}\left(p^{\prime}, s_{p}^{\prime}\right) \Gamma_{\nu}\left(p^{\prime}, p\right) N\left(p, s_{p}\right) \\
& \times \bar{u}\left(k^{\prime}, h^{\prime}\right) \gamma^{\nu} \frac{\left(\not k-\not q^{\prime}+m\right)}{-2 k \cdot q^{\prime}} \tilde{\Sigma}\left(k-q^{\prime}\right) \frac{\left(\not k-\not q^{\prime}+m\right)}{-2 k \cdot q^{\prime}} \xi^{*} u(k, h),
\end{aligned}
$$

where the renormalized self-energy is denoted by $\tilde{\Sigma}$ and is given by Eq. (A30). Remark that the UV divergence in the loop integral of Fig. 2 ( $\mathrm{Si}$ ) has been removed through the renormalization of the electron field and electron mass. The UV finite renormalized selfenergy $\tilde{\Sigma}$ contains however an IR divergence from the counterterms. Inserting the expression for $\tilde{\Sigma}$ (Eq. (A30)) into Eq.(37), yields :

$$
\begin{aligned}
M_{S i}= & M_{B H}^{i} \frac{e^{2}}{(4 \pi)^{2}} 2\left[\frac{1}{\varepsilon_{I R}}-\gamma_{E}+\ln \left(\frac{4 \pi \mu^{2}}{m^{2}}\right)\right] \\
+ & \frac{i e^{5}}{(4 \pi)^{2}} \frac{1}{\left(p^{\prime}-p\right)^{2}} \bar{N}\left(p^{\prime}, s_{p}^{\prime}\right) \Gamma_{\nu}\left(p^{\prime}, p\right) N\left(p, s_{p}\right) \\
& \times \bar{u}\left(k^{\prime}, h^{\prime}\right) \gamma^{\nu} \frac{\left(\not k-\not q^{\prime}+m\right)}{-2 k \cdot q^{\prime}}\left\{\frac{m\left(\not k-\not q^{\prime}\right)}{m^{2}-2 k \cdot q^{\prime}}\left[1+\frac{-2 m^{2}+6 k \cdot q^{\prime}}{m^{2}-2 k \cdot q^{\prime}} \ln \left(\frac{2 k \cdot q^{\prime}}{m^{2}}\right)\right]\right. \\
& \left.+\left[3-\frac{2 m^{2}+2 k \cdot q^{\prime}}{m^{2}-2 k \cdot q^{\prime}} \ln \left(\frac{2 k \cdot q^{\prime}}{m^{2}}\right)\right]\right\} \xi^{*} u(k, h) .
\end{aligned}
$$

The amplitude corresponding to Fig. 2 (Sf) is given by :

$$
\begin{aligned}
M_{S f} & =i e^{3} \frac{1}{\left(p^{\prime}-p\right)^{2}} \bar{N}\left(p^{\prime}, s_{p}^{\prime}\right) \Gamma_{\nu}\left(p^{\prime}, p\right) N\left(p, s_{p}\right) \\
& \times \bar{u}\left(k^{\prime}, h^{\prime}\right) k^{*} \frac{\left(\not k^{\prime}+\not q^{\prime}+m\right)}{2 k^{\prime} \cdot q^{\prime}} \tilde{\Sigma}\left(k^{\prime}+q^{\prime}\right) \frac{\left(\not k^{\prime}+\not q^{\prime}+m\right)}{2 k \cdot q^{\prime}} \gamma^{\nu} u(k, h),
\end{aligned}
$$

which can be worked out analogously as before and yields :

$$
\begin{aligned}
M_{S f}= & M_{B H}^{f} \frac{e^{2}}{(4 \pi)^{2}} 2\left[\frac{1}{\varepsilon_{I R}}-\gamma_{E}+\ln \left(\frac{4 \pi \mu^{2}}{m^{2}}\right)\right] \\
+ & \frac{i e^{5}}{(4 \pi)^{2}} \frac{1}{\left(p^{\prime}-p\right)^{2}} \bar{N}\left(p^{\prime}, s_{p}^{\prime}\right) \Gamma_{\nu}\left(p^{\prime}, p\right) N\left(p, s_{p}\right) \\
& \times \bar{u}\left(k^{\prime}, h^{\prime}\right) k^{*} \frac{\left(\not k^{\prime}+\not q^{\prime}+m\right)}{2 k^{\prime} \cdot q^{\prime}}\left\{\frac{m\left(\not k^{\prime}+\not q^{\prime}\right)}{m^{2}+2 k^{\prime} \cdot q^{\prime}}\left[1+\frac{-2 m^{2}-6 k^{\prime} \cdot q^{\prime}}{m^{2}+2 k^{\prime} \cdot q^{\prime}} \ln \left(\frac{-2 k^{\prime} \cdot q^{\prime}}{m^{2}}\right)\right]\right. \\
& \left.+\left[3-\frac{2 m^{2}-2 k^{\prime} \cdot q^{\prime}}{m^{2}+2 k^{\prime} \cdot q^{\prime}} \ln \left(\frac{-2 k^{\prime} \cdot q^{\prime}}{m^{2}}\right)\right]\right\} \gamma^{\nu} u(k, h) .
\end{aligned}
$$


Note that in Fig. 2 (Sf), the four-momentum squared of the $\left(e^{-}+\gamma^{*}\right)$ state in the loop is given by $\left(k^{\prime}+q^{\prime}\right)^{2}=m^{2}+2 k^{\prime} \cdot q^{\prime} \geq m^{2}$. Therefore, the self-energy and the amplitude for Fig. $\mathrm{Z}(\mathrm{Sf})$ is complex, as was also noted for the vertex diagrams of Fig. 2 where the photon is emitted from the final electron (denoted by $f$ ). Eq. (40) yields indeed a complex amplitude because $\ln \left(-2 k^{\prime} \cdot q^{\prime} / m^{2}\right)=\ln \left(2 k^{\prime} \cdot q^{\prime} / m^{2}\right)+i \pi$, for $k^{\prime} \cdot q^{\prime}>0$.

\section{Vertex correction diagram of Fig. 国(V4)}

The vertex correction to the VCS process is given by Fig. 2 (V4), and its calculation is the same as the one for elastic electron scattering. This yields for the renormalized vertex correction :

$$
M_{V 4}=-i e^{3} \bar{u}\left(k^{\prime}, h^{\prime}\right)\left[\left(F\left(Q^{2}\right)-F\left(Q^{2}=0\right)\right) \gamma_{\nu}-G\left(Q^{2}\right) i \sigma_{\nu \kappa} \frac{q^{\kappa}}{2 m}\right] u(k, h) \frac{1}{q^{2}} \varepsilon_{\mu}^{*} H^{\mu \nu} .
$$

In Eq. (41), $F\left(Q^{2}\right)-F\left(Q^{2}=0\right)$ is given by Eq. (A17) and reduces in the ultrarelativistic limit $\left(Q^{2}>>m^{2}\right)$ to Eq. (A19). The magnetic correction $G\left(Q^{2}\right)$ is given by Eq. (A11), and vanishes in the ultrarelativistic limit.

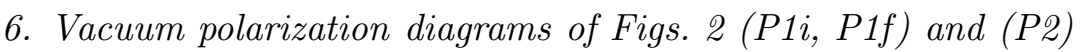

The vacuum polarization corrections of Figs. 2 (P1i, P1f) and (P2) involve the renormalized photon self-energy $\tilde{\Pi}\left(Q^{2}\right)$, which has been calculated in appendix $\mathrm{A}$. Therefore, we get for the vacuum polarization correction to the BH process (Figs. 2 (P1i, P1f)) :

$$
M_{P 1}^{i}=M_{B H}^{i} \frac{1}{1-\tilde{\Pi}(-t)}, \quad \text { and } \quad M_{P 1}^{f}=M_{B H}^{f} \frac{1}{1-\tilde{\Pi}(-t)},
$$

with $t=\left(p^{\prime}-p\right)^{2}$. Similarly, we get for the vacuum polarization correction to the VCS process (Fig. $2(\mathrm{P} 2))$ :

$$
M_{P 2}=M_{V C S} \frac{1}{1-\tilde{\Pi}\left(Q^{2}\right)} .
$$

In the ultrarelativistic limit $\left(Q^{2}>>m^{2}\right), \tilde{\Pi}\left(Q^{2}\right)$ is obtained from Eq. (A40)

$$
\tilde{\Pi}\left(Q^{2}\right)=\frac{e^{2}}{(4 \pi)^{2}} \frac{4}{3}\left\{-\frac{5}{3}+\ln \left(\frac{Q^{2}}{m^{2}}\right)\right\} \text {. }
$$

\section{B. Soft-photon emission contributions and cancellation of IR divergences}

After removing the UV divergences from the virtual photon corrections to the $e p \rightarrow e p \gamma$ reaction in the last section, the resulting expressions still contain IR divergences. Both the corrections to the BH process of Figs. 2 (V1i, V1f, V2i, V2f, V3i, V3f, Si and Sf) and the vertex correction of Fig. 2 (V4) to the VCS process contain IR divergences. It is known 
for QED since a long time [26,27], that these IR divergences are cancelled at the cross section level by soft photon emission contributions. These soft photons are emitted from the charged particle lines and can have energies up to some maximal value $\Delta E_{s}$ which is related to the finite resolution of the detector. In appendix A (section A5), we calculate the soft bremsstrahlung contribution to electron scattering by performing the phase space integral over the soft photon in an exact way, and give the finite correction (after cancellation of all IR divergences) to the elastic electron scattering cross section. In this section, we generalize the result of appendix $\mathrm{A}$ to the case of the $e p \rightarrow e p \gamma$ reaction. The diagrams for the $e p \rightarrow e p \gamma$ reaction with one additional soft photon are shown in Fig. 3, where the hard photon of the $e p \rightarrow e p \gamma$ process is indicated by its four-momentum $q^{\prime}$. In this section, we will show that the soft photon emission contributions of Fig. 3 contain IR divergences which exactly cancel the IR divergences appearing in the virtual photon correction diagrams of Fig. 2. The process where the energy $\Delta E_{s}$ of the additionally emitted photon is not very small compared with the lepton momenta in the process, makes up the radiative tail to the $e p \rightarrow e p \gamma$ reaction. Its calculation will be discussed in section IV.

\section{Factorization of amplitude for soft-photon emission processes}

Here, we evaluate the diagrams of Fig. 3 in the soft photon limit, i.e. when the second emitted photon has an energy much smaller than the initial and final lepton energies and also smaller than the hard photon (denoted by $q^{\prime}$ ) in order to distinguish both photons. We will see that only the diagrams where a soft photon couples to an on-shell lepton contain IR divergences and lead to a finite logarithmic correction in $\Delta E_{s}$.

The amplitude corresponding with Fig. 3 (b1i) is given by :

$$
\begin{aligned}
M_{b 1 i}= & i e^{3} \bar{u}\left(k^{\prime}, h^{\prime}\right) \gamma^{\nu} \frac{\left(\not k-\not q^{\prime}-\not l+m\right)}{-2 k \cdot q^{\prime}-2 l .\left(k-q^{\prime}\right)} k^{*}\left(q^{\prime}\right) \frac{(\not k-\not l+m)}{-2 k . l}\left(-e \not k^{*}(l)\right) u(k, h) \\
& \times \frac{1}{\left(p^{\prime}-p\right)^{2}} \bar{N}\left(p^{\prime}, s_{p}^{\prime}\right) \Gamma_{\nu}\left(p^{\prime}, p\right) N\left(p, s_{p}\right),
\end{aligned}
$$

where $l$ is the four-momentum of the soft photon. In the soft photon limit $(l \rightarrow 0)$, Eq. (45) simplifies by using $(\not k-\not l+m) \gamma^{\alpha} u(k, h)=\left(2 k^{\alpha}-\not \gamma^{\alpha}\right) u(k, h) \approx 2 k^{\alpha} u(k, h)$, which yields for Eq. (45) in the soft photon limit :

$$
M_{b 1 i}=M_{B H}^{i}(-e) \varepsilon_{\alpha}^{*}(l)\left[-\frac{k^{\alpha}}{k . l}\right],
$$

where $M_{B H}^{i}$ is the Bethe-Heitler amplitude of Eq. (1) - corresponding with photon emission from the initial lepton. Similarly, we can derive the amplitude for Figs. 3 (b2i, b1f and b2f) which yields in the soft photon limit :

$$
\begin{aligned}
M_{b 1 i}+M_{b 2 i} & =M_{B H}^{i}(-e) \varepsilon_{\alpha}^{*}(l)\left[\frac{k^{\prime \alpha}}{k^{\prime} . l}-\frac{k^{\alpha}}{k . l}\right], \\
M_{b 1 f}+M_{b 2 f} & =M_{B H}^{f}(-e) \varepsilon_{\alpha}^{*}(l)\left[\frac{k^{\prime \alpha}}{k^{\prime} . l}-\frac{k^{\alpha}}{k . l}\right],
\end{aligned}
$$


where $M_{B H}^{f}$ is the Bethe-Heitler amplitude of Eq. (2) - corresponding with photon emission from the final lepton.

Figs. 3 (b3i) and (b3f) contain the contributions where the soft photon couples to an off-shell lepton line. The amplitude corresponding with Fig. 3 (b3i) is given by :

$$
\begin{aligned}
M_{b 3 i}= & -i e^{4} \bar{u}\left(k^{\prime}, h^{\prime}\right) \gamma^{\nu} \frac{\left(\not k-\not q^{\prime}-\not l+m\right)}{-2 k \cdot q^{\prime}-2 l .\left(k-q^{\prime}\right)} k^{*}(l) \frac{\left(\not k-\not q^{\prime}+m\right)}{-2 k \cdot q^{\prime}} k^{*}\left(q^{\prime}\right) u(k, h) \\
& \times \frac{1}{\left(p^{\prime}-p\right)^{2}} \bar{N}\left(p^{\prime}, s_{p}^{\prime}\right) \Gamma_{\nu}\left(p^{\prime}, p\right) N\left(p, s_{p}\right),
\end{aligned}
$$

In the soft photon limit, Eq. (49) can be simplified by using :

$$
\begin{aligned}
\frac{\left(\not k-\not q^{\prime}-\not \not+m\right)}{-2 k \cdot q^{\prime}-2 l \cdot\left(k-q^{\prime}\right)} \gamma^{\alpha} \frac{\left(\not k-\not q^{\prime}+m\right)}{-2 k \cdot q^{\prime}} & \approx \frac{\left(\not k-\not q^{\prime}+m\right)}{-2 k \cdot q^{\prime}} \gamma^{\alpha} \frac{\left(\not k-\not q^{\prime}+m\right)}{-2 k \cdot q^{\prime}} \\
& =\frac{\left(\not k-\not q^{\prime}+m\right)}{-2 k \cdot q^{\prime}} \frac{\left(k-q^{\prime}\right)^{\alpha}}{-k \cdot q^{\prime}}-\frac{\gamma^{\alpha}}{-2 k \cdot q^{\prime}} .
\end{aligned}
$$

Consequently, the amplitude of Eq. (49) is given by :

$$
\begin{aligned}
M_{b 3 i} & =M_{B H}^{i}(-e) \varepsilon_{\alpha}^{*}(l) \frac{\left(k-q^{\prime}\right)^{\alpha}}{-k \cdot q^{\prime}} \\
& +i e^{4} \bar{u}\left(k^{\prime}, h^{\prime}\right) \frac{\gamma^{\nu} \xi^{*}(l) k^{*}\left(q^{\prime}\right)}{-2 k \cdot q^{\prime}} u(k, h) \frac{1}{\left(p^{\prime}-p\right)^{2}} \bar{N}\left(p^{\prime}, s_{p}^{\prime}\right) \Gamma_{\nu}\left(p^{\prime}, p\right) N\left(p, s_{p}\right) .
\end{aligned}
$$

Similarly, the amplitude corresponding with Fig. 3 (b3f) is given by :

$$
\begin{aligned}
M_{b 3 f} & =M_{B H}^{f}(-e) \varepsilon_{\alpha}^{*}(l) \frac{\left(k^{\prime}+q^{\prime}\right)^{\alpha}}{k^{\prime} \cdot q^{\prime}} \\
& +i e^{4} \bar{u}\left(k^{\prime}, h^{\prime}\right) \frac{\xi^{*}\left(q^{\prime}\right) \xi^{*}(l) \gamma^{\nu}}{2 k^{\prime} \cdot q^{\prime}} u(k, h) \frac{1}{\left(p^{\prime}-p\right)^{2}} \bar{N}\left(p^{\prime}, s_{p}^{\prime}\right) \Gamma_{\nu}\left(p^{\prime}, p\right) N\left(p, s_{p}\right) .
\end{aligned}
$$

In complete analogy to Eqs. (47,48), we can also calculate the soft photon emission contributions to the VCS process. They are shown in Figs. 3 (b4) and (b5), and their calculation in the soft photon limit yields :

$$
M_{b 4}+M_{b 5}=M_{V C S}(-e) \varepsilon_{\alpha}^{*}(l)\left[\frac{k^{\prime \alpha}}{k^{\prime} . l}-\frac{k^{\alpha}}{k . l}\right],
$$

where $M_{V C S}$ is the VCS amplitude of Eq. (里).

We see from Eqs. (47,48 and 53), that for the diagrams of Fig. 3 where the soft photon couples to an on-shell lepton, the original amplitude factorizes : in Eqs. (47,48) the BH amplitude factorizes, and in Eq. (53) the VCS amplitude factorizes. The resulting amplitudes are proportional to $1 / l$, which leads to a logarithmic divergence when integrating over the phase space of the soft photon. In contrast, the amplitudes of Eqs. (51,52) where the photon couples to an off-shell lepton line are finite when $l \rightarrow 0$, and the corresponding phase space integral becomes vanishingly small in the limit $l \rightarrow 0$. 


\section{Radiative correction due to soft-photon emission processes}

In the soft-photon limit, we therefore need only to keep the bremsstrahlung corrections of Eqs. (47,48 and 53), where the BH and VCS amplitudes factorize. To first order in $\alpha_{e m}$ (relative to the $\mathrm{BH}+\mathrm{VCS}$ cross section) the bremsstrahlung correction therefore amounts to calculate the phase space integral of the form :

$$
\begin{aligned}
d \sigma \sim \frac{d^{3} \vec{k}_{e}^{\prime}}{(2 \pi)^{3} 2 E_{e}^{\prime}} \frac{d^{3} \vec{q}^{\prime}}{(2 \pi)^{3} 2\left|\vec{q}^{\prime}\right|} & \frac{d^{3} \vec{p}_{N}^{\prime}}{(2 \pi)^{3} 2 E_{N}^{\prime}} \frac{d^{3} \vec{l}}{(2 \pi)^{3} 2 l}(2 \pi)^{4} \delta^{4}\left(k+p-k^{\prime}-q^{\prime}-p^{\prime}-l\right) \\
& \times\left|M_{B H}+M_{V C S}\right|^{2}\left(-e^{2}\right)\left[\frac{k_{\mu}^{\prime}}{k^{\prime} . l}-\frac{k_{\mu}}{k . l}\right] \cdot\left[\frac{k^{\prime \mu}}{k^{\prime} . l}-\frac{k^{\mu}}{k . l}\right],
\end{aligned}
$$

where $l \equiv|\vec{l}|$ denotes the soft photon energy, and where the total $\mathrm{BH}$ amplitude is given by $M_{B H}=M_{B H}^{i}+M_{B H}^{f}$. The calculation of the bremsstrahlung integral of Eq. (54) goes along similar lines as the corresponding integral for elastic scattering, for which the technical details can be found in appendix A (section A5). We will point out in this section the differences which arise for the $e p \rightarrow e p \gamma$ reaction.

There are two practical ways to measure the $e p \rightarrow e p \gamma$ reaction, by measuring two particles in the final state. One can either measure the outgoing electron in coincidence with the recoiling nucleon : this is the ideal technique when measuring the $e p \rightarrow e p \gamma$ reaction at low outgoing photon energy as is done in [11 [13]. The alternative is to measure the outgoing electron in coincidence with the photon : this is the technique when doing a very inelastic experiment, such as deeply virtual Compton scattering, where the photon is produced with a large energy. We discuss here first the case where one detects the outgoing electron and photon, and indicate at the end the changes which apply when measuring the outgoing electron and recoiling nucleon.

If one measures the $e p \rightarrow e p \gamma$ reaction by detecting the outgoing electron and photon, one eliminates in Eq. (A41) the integral over $\vec{p}_{N}^{\prime}$ with the momentum conserving $\delta$-function, which gives :

$$
\begin{aligned}
d \sigma \sim & \frac{d^{3} \vec{k}_{e}^{\prime}}{(2 \pi)^{3} 2 E_{e}^{\prime}} \frac{d^{3} \vec{q}^{\prime}}{(2 \pi)^{3} 2\left|\vec{q}^{\prime}\right|} \frac{d^{3} \vec{l}}{(2 \pi)^{3} 2 l} \frac{1}{2 E_{N}^{\prime}} \\
& \times(2 \pi) \delta\left(E_{e}+E_{N}-E_{e}^{\prime}-\left|\vec{q}^{\prime}\right|-\sqrt{\left(\vec{q}+\vec{p}_{N}-\vec{q}^{\prime}-\vec{l}\right)^{2}+M_{N}^{2}}-1\right) \\
& \times\left|M_{B H}+M_{V C S}\right|^{2}\left(-e^{2}\right)\left[\frac{k_{\mu}^{\prime}}{k^{\prime} . l}-\frac{k_{\mu}}{k . l}\right] \cdot\left[\frac{k^{\prime \mu}}{k^{\prime} . l}-\frac{k^{\mu}}{k . l}\right] .
\end{aligned}
$$

Due to the energy conserving $\delta$-function in Eq. (A42), the upper limit in the integration over the soft photon phase space depends on the angle. Therefore, this integration volume has a complicated ellipsoidal shape in the lab system. In order for the soft-photon phase space integration volume to be spherical, one has to perform the calculation in the c.m. system $\mathcal{S}_{1}$ of the (recoiling nucleon + soft-photon), generalizing the procedure of appendix A for elastic scattering to the $e p \rightarrow e p \gamma$ reaction. The system $\mathcal{S}_{1}$ is defined by $: \vec{p}_{N}^{\prime}+\vec{l}=\vec{p}_{N}+\vec{q}-\vec{q}^{\prime}=0$. 
In the system $\mathcal{S}_{1}$, the energy conserving delta function in Eq. (55) is independent of the softphoton angles, and the maximal soft photon energy is isotropic. The integral over the softphoton momentum (up to some maximum value $\Delta E_{s}$ ) can then be performed independently from the integration over the soft photon emission angles. If $\Delta E_{s}$ is sufficiently small, one can furthermore neglect the soft photon energy with respect to the other energies in the $\delta$ function, and perform the integral over the photon momentum $\left|\vec{q}^{\prime}\right|$ in Eq. (55) to obtain the correction to the fivefold differential $e p \rightarrow e p \gamma$ cross section. We indicate in the following only how the squared matrix element for the $e p \rightarrow e p \gamma$ reaction is modified due to soft photon emission. This correction due to soft bremsstrahlung is given by :

$$
\left|M_{e p \rightarrow e p \gamma}^{S O F T \gamma}\right|^{2}=\left|M_{B H}+M_{V C S}\right|^{2}\left(-e^{2}\right) \int \frac{d^{3} \vec{l}}{(2 \pi)^{3} 2 l}\left[\frac{k_{\mu}^{\prime}}{k^{\prime} . l}-\frac{k_{\mu}}{k . l}\right] \cdot\left[\frac{k^{\prime \mu}}{k^{\prime} . l}-\frac{k^{\mu}}{k . l}\right] .
$$

The correction factor multiplying $\left|M_{B H}+M_{V C S}\right|^{2}$ gives immediately the correction factor to the fivefold $e p \rightarrow e p \gamma$ cross section. In Eq. (56), the soft-photon phase space integral is understood to be performed in the system $\mathcal{S}_{1}$, where the integration volume is spherical. Its calculation was already performed in appendix A. One sees that the integral in Eq. (56) has a logarithmic IR divergence, corresponding with the emission of photons with zero energy. To evaluate it, one has to regularize it, which is done in this work by using dimensional regularization. This amounts to evaluate the integral (in the system $\mathcal{S}_{1}$ ) in $D-1$ dimensions ( $D \rightarrow 4$ corresponds to the physical limit). This calculation is performed in appendix $\mathrm{A}$ and yields (similar to Eq. (A63)) as result :

$$
\begin{aligned}
\left|M_{e p \rightarrow e p \gamma}^{S O F T \gamma}\right|^{2} & =\left|M_{B H}+M_{V C S}\right|^{2} \\
& \times\left\{\frac{e^{2}}{4 \pi^{2}}\left[-\frac{1}{\varepsilon_{I R}}+\gamma_{E}-\ln \left(\frac{4 \pi \mu^{2}}{m^{2}}\right)\right]\left[\frac{v^{2}+1}{2 v} \ln \left(\frac{v+1}{v-1}\right)-1\right]+\delta_{R}\right\},
\end{aligned}
$$

In Eq. (57), $\delta_{R}$ is the finite part of the real radiative correction corresponding with soft photon emission, and is given as in appendix A (Eq. (A66)) by :

$$
\begin{aligned}
\delta_{R} \stackrel{Q^{2} \gg m^{2}}{\longrightarrow} \frac{\alpha_{e m}}{\pi} & \left\{\ln \left(\frac{\left(\Delta E_{s}\right)^{2}}{\tilde{E}_{e} \tilde{E}_{e}^{\prime}}\right)\left[\ln \left(\frac{Q^{2}}{m^{2}}\right)-1\right]\right. \\
& \left.-\frac{1}{2} \ln ^{2}\left(\frac{\tilde{E}_{e}}{\tilde{E}_{e}^{\prime}}\right)+\frac{1}{2} \ln ^{2}\left(\frac{Q^{2}}{m^{2}}\right)-\frac{\pi^{2}}{3}+S p\left(\cos ^{2} \frac{\tilde{\theta}_{e}}{2}\right)\right\},
\end{aligned}
$$

In Eq. (58), we next have to express the kinematical variables $\left(\tilde{E}_{e}, \tilde{E}_{e}^{\prime}, \cos \tilde{\theta}_{e}\right)$ in the system $\mathcal{S}_{1}$ (denoted by tilded quantities) in terms of the lab quantities, which we denote by untilded quantities $\left(E_{e}, E_{e}^{\prime}, \cos \theta_{e}\right)$. To make the transformation between the system $\mathcal{S}_{1}$ and the $l a b$ system, we first introduce the missing four-momentum $p_{m 1} \equiv p_{N}^{\prime}+l$. The system $\mathcal{S}_{1}$ is defined by $\vec{p}_{m 1}=\overrightarrow{0}$, and the missing mass $M_{m 1}$ of the system $\left(p^{\prime}+l\right)$ is defined by :

$$
M_{m 1}^{2}=\left(p^{\prime}+l\right)^{2}=\left(p+q-q^{\prime}\right)^{2} .
$$

We can then easily express the electron energies and angle in the system $\mathcal{S}_{1}$ in terms of $l a b$ quantities : 


$$
\begin{aligned}
& \tilde{E}_{e}=\frac{k \cdot p_{m 1}}{M_{m 1}}=\frac{1}{M_{m 1}} k \cdot\left(p+q-q^{\prime}\right)=\frac{M_{N}}{M_{m 1}}\left(E_{e}-\frac{Q^{2}}{2 M_{N}}-\frac{k \cdot q^{\prime}}{M_{N}}\right), \\
& \tilde{E}_{e}^{\prime}=\frac{k^{\prime} \cdot p_{m 1}}{M_{m 1}}=\frac{1}{M_{m 1}} k^{\prime} \cdot\left(p+q-q^{\prime}\right)=\frac{M_{N}}{M_{m 1}}\left(E_{e}^{\prime}+\frac{Q^{2}}{2 M_{N}}-\frac{k^{\prime} \cdot q^{\prime}}{M_{N}}\right), \\
& \sin ^{2} \tilde{\theta}_{e} / 2=\frac{E_{e} E_{e}^{\prime}}{\tilde{E}_{e} \tilde{E}_{e}^{\prime}} \sin ^{2} \theta_{e} / 2 .
\end{aligned}
$$

The maximal soft-photon energy $\Delta E_{s}$ in the system $\mathcal{S}_{1}$, is given by :

$$
\Delta E_{s}=\frac{M_{m 1}^{2}-M_{N}^{2}}{2 M_{m 1}} .
$$

If one measures the $e p \rightarrow e p \gamma$ reaction by detecting the outgoing electron and recoiling proton, the derivation goes along similar lines as above. One starts now by eliminating in Eq. (A41) the integral over $\vec{q}^{\prime}$. Then one goes into the c.m. system $\mathcal{S}_{2}$ of the (VCS photon $q^{\prime}+$ soft photon), where the energy conserving $\delta$-function is independent of the soft-photon angles, and where the maximal soft photon energy is isotropic. This system $\mathcal{S}_{2}$ is defined by : $\vec{q}^{\prime}+\vec{l}=\vec{p}_{N}+\vec{q}-\vec{p}_{N}^{\prime}=0$. The calculation of the soft-photon emission integral is then completely similar as above, and leads to the finite correction of Eq.(58), where the the kinematical variables $\left(\tilde{E}_{e}, \tilde{E}_{e}^{\prime}, \cos \tilde{\theta}_{e}\right)$ are now understood in the system $\mathcal{S}_{2}$. To make the transformation between the system $\mathcal{S}_{2}$ and the lab system, we first introduce the missing four-momentum $p_{m 2} \equiv q^{\prime}+l$. The system $\mathcal{S}_{2}$ is defined by $\vec{p}_{m 2}=\overrightarrow{0}$, and the missing mass $M_{m 2}$ of the system $\left(q^{\prime}+l\right)$ is defined by :

$$
M_{m 2}^{2}=\left(q^{\prime}+l\right)^{2}=\left(p+q-p^{\prime}\right)^{2} .
$$

We can then easily express the electron energies and angle in the system $\mathcal{S}_{2}$ in terms of lab quantities :

$$
\begin{aligned}
& \tilde{E}_{e}=\frac{k \cdot p_{m 2}}{M_{m 2}}=\frac{1}{M_{m 2}} k \cdot\left(p+q-p^{\prime}\right)=\frac{M_{N}}{M_{m 2}}\left(E_{e}-\frac{Q^{2}}{2 M_{N}}-\frac{k \cdot p^{\prime}}{M_{N}}\right) \\
& \tilde{E}_{e}^{\prime}=\frac{k^{\prime} \cdot p_{m 2}}{M_{m 2}}=\frac{1}{M_{m 2}} k^{\prime} \cdot\left(p+q-p^{\prime}\right)=\frac{M_{N}}{M_{m 2}}\left(E_{e}^{\prime}+\frac{Q^{2}}{2 M_{N}}-\frac{k^{\prime} \cdot p^{\prime}}{M_{N}}\right), \\
& \sin ^{2} \tilde{\theta}_{e} / 2=\frac{E_{e} E_{e}^{\prime}}{\tilde{E}_{e} \tilde{E}_{e}^{\prime}} \sin ^{2} \theta_{e} / 2 .
\end{aligned}
$$

The maximal soft-photon energy $\Delta E_{s}$ in the system $\mathcal{S}_{2}$, is given by :

$$
\Delta E_{s}=\frac{M_{m 2}}{2}
$$

\section{Cancellation of IR divergences}

We can now demonstrate for the $e p \rightarrow e p \gamma$ reaction, that the IR divergences from the soft photon emission corrections exactly cancel against the IR divergences from the virtual radiative corrections, calculated in section III A. Concentrating here only on the IR divergent 
parts of the virtual radiative corrections, we found in section IIIA that the amplitudes of Eqs. (13, 17, 22, 24, 33, 34, 38,40, and 41) contain IR divergences. Those IR divergent parts are given by :

$$
\begin{aligned}
& M_{V 1}^{i}+(C T)_{V 1}^{i}+M_{V 1}^{f}+(C T)_{V 1}^{f} \rightarrow M_{B H} \frac{e^{2}}{4 \pi^{2}}\left(\frac{-1}{2}\right)\left[\frac{1}{\varepsilon_{I R}}-\gamma_{E}+\ln \left(\frac{4 \pi \mu^{2}}{m^{2}}\right)\right] \\
& M_{V 2}^{i}+(C T)_{V 2}^{i}+M_{V 2}^{f}+(C T)_{V 2}^{f} \rightarrow M_{B H} \frac{e^{2}}{4 \pi^{2}}\left(\frac{-1}{2}\right)\left[\frac{1}{\varepsilon_{I R}}-\gamma_{E}+\ln \left(\frac{4 \pi \mu^{2}}{m^{2}}\right)\right] \\
& M_{V 3}^{i}+M_{V 3}^{f} \rightarrow M_{B H} \frac{e^{2}}{4 \pi^{2}}\left(\frac{1}{2}\right) \frac{v^{2}+1}{2 v} \ln \left(\frac{v+1}{v-1}\right)\left[\frac{1}{\varepsilon_{I R}}-\gamma_{E}+\ln \left(\frac{4 \pi \mu^{2}}{m^{2}}\right)\right] \\
& M_{S i}+M_{S f} \rightarrow M_{B H} \frac{e^{2}}{4 \pi^{2}}\left(\frac{1}{2}\right)\left[\frac{1}{\varepsilon_{I R}}-\gamma_{E}+\ln \left(\frac{4 \pi \mu^{2}}{m^{2}}\right)\right] \\
& M_{V 4}+(C T)_{V 4} \rightarrow M_{V C S} \frac{e^{2}}{4 \pi^{2}}\left(\frac{1}{2}\right)\left[\frac{v^{2}+1}{2 v} \ln \left(\frac{v+1}{v-1}\right)-1\right]\left[\frac{1}{\varepsilon_{I R}}-\gamma_{E}+\ln \left(\frac{4 \pi \mu^{2}}{m^{2}}\right)\right] .
\end{aligned}
$$

Adding them all up gives the following correction to the squared amplitude for the virtual radiative corrections :

$$
\begin{aligned}
& \left|M_{B H}+M_{D V C S}+M_{e p \rightarrow e p \gamma}^{V I R T U A \gamma}\right|^{2} \\
& =\left|M_{B H}+M_{V C S}\right|^{2}\left\{1+\frac{e^{2}}{4 \pi^{2}}\left[\frac{v^{2}+1}{2 v} \ln \left(\frac{v+1}{v-1}\right)-1\right]\left[\frac{1}{\varepsilon_{I R}}-\gamma_{E}+\ln \left(\frac{4 \pi \mu^{2}}{m^{2}}\right)\right]\right\}+\ldots
\end{aligned}
$$

where the ellipses denote the finite first order virtual radiative correction to the $e p \rightarrow e p \gamma$ reaction as was calculated and can be found in section IIIA. Adding the virtual (Eq.(74)) and real (Eq.(57)) radiative corrections to the $e p \rightarrow e p \gamma$ reaction, one verifies that the IR divergences in the sum exactly cancel, showing QED at work! Note that this cancellation is different as compared to the case of elastic electron scattering. Indeed, for the virtual photon correction diagrams to the Bethe Heitler process, there are 3 types of vertex diagrams (Eqs. (69,70 and 71)) and the self energy diagram (Eq. (72)), and the corresponding counterterms, which have an IR divergence. On the other hand, for the virtual radiative corrections to elastic electron scattering, there is only one vertex diagram which is IR divergent.

\section{Integration method for the virtual photon corrections}

At this stage of the calculation of the first order QED radiative corrections to the $e p \rightarrow e p \gamma$ reaction, the treatment of all UV and IR divergences, resulting from the radiative corrections at the electron side, has been performed. The UV divergences have been removed by the renormalization procedure whereas the IR divergences were shown to cancel at the cross section level when adding the soft photon emission processes. Now, the evaluation of the remaining Feynman parameter integrals in the finite terms such as in Eq. (13) still has to be done.

Among the one-loop virtual radiative corrections to the $e p \rightarrow e p \gamma$ reaction shown in Fig. 2, six give rise to simple analytical formulas. For the six vertex diagrams, denoted by V1i,V2i,V3i,V1f,V2f and V3f, the trick consisting of adding and subtracting the divergent 
term for each of them (as explained in Section IIIA) gives rise to Feynman parameter integrals that are rather complicated to be done analytically. Therefore, we will evaluate them in this work by a numerical procedure. Although these Feynman parameter integrals are by construction finite, appropriate numerical methods are needed to perform them. Two main difficulties are encountered in these numerical integrations. Firstly, the variations of the integrated functions are always extremely sharp near the integration limits. In fact, a typical behaviour is a rather flat dependence in the middle of the domain and two pronounced rises when approaching 0 or 1 for the Feynman parameters with a width of the order $m / E_{e}$. The contribution of these two peaks has to be evaluated carefully in order to obtain a good precision for the final result. Secondly, we know that the virtual radiative corrections to the $e p \rightarrow e p \gamma$ reaction allow the propagation of on-shell states (see section III A). This is mathematically expressed by the presence of integrable singularities in the Feynman parameter integrals which require an analytical continuation into the complex plane and gives rise to an imaginary part for the amplitude.

To evaluate the Feynman parameter integrals, our strategy is to perform the first integration analytically. The last integrations will then be performed numerically using the Gauss-Legendre integration routine. The analytical calculation of the first integration provides a shorter calculational time and a higher precision. The main advantage however is that in the case of a singularity, the pole is avoided by deforming the integration contour into the complex plane, using analytical continuation. In this way, one removes the difficulties for the remaining integrations along the real axis.

To classify the Feynman parameter integrals that occur in the six vertex diagrams under study, we start by factorizing all the Dirac $\gamma$ matrices and decomposing the components of the four-vectors. All resulting integrals then reduce to the generic form :

$$
\iiint_{0}^{1} d x_{1} d x_{2} d x_{3} \frac{P\left(x_{1}, x_{2}, x_{3}\right)}{Q\left(x_{1}, x_{2}, x_{3}\right)},
$$

where $P$ and $Q$ are polynomials in three (real) Feynman parameters $x_{1}, x_{2}, x_{3}$. Let's choose $x_{1}$ to be the more internal variable. Then the first integration is either of the form :

$$
\int_{0}^{1} \frac{x_{1}^{m} d x_{1}}{\left(\alpha x_{1}+\beta\right)^{n}}
$$

or

$$
\int_{0}^{1} \frac{x_{1}^{m} d x_{1}}{\left(\alpha x_{1}^{2}+\beta x_{1}+\gamma\right)^{n}},
$$

where $\alpha, \beta$ and $\gamma$ are polynomials in $x_{2}$ and $x_{3}$ with coefficients that are functions of kinematical variables. In Eqs. (76,77), $m$ varies from 0 to 4 and $n$ is equal to 1 or 2, to accomodate all cases appearing in section [IIA. These successive decompositions increase the number of terms to calculate but they have the advantage to provide two simple classes of integrals without any vector or matrix dependence. The possibility of poles in the integrands of Eqs. (76,77) naturally splits the problem into two parts, whether the integrand is regular or singular. 


\section{Regular integrand}

When the denominator doesn't have any singularities, some recurrence relations exist for these integrals and can be found in Ref. [28]. Unfortunately for small values of $\alpha$ as compared to $\beta$ or to $\gamma$, it has been seen that these relations are numerically unstable. This has thus led us to use several methods of integration with each a different domain of validity. For small ratios $r(r=\alpha / \beta$ for Eq. (76) or $r=\alpha / \gamma$ for Eq. (77) $)$ as compared to 1, we perform a Taylor expansion of the integral and tune the order of each development to complete a fixed criterium of convergence (for example we require that the ratio between the last and the first terms is of the order of the numerical precision in double precision). For $r>1$ the recurrence relations [28] are used as they are stable in this range. In the intermediate zone $(0.2 \leq r \leq 1)$, we use the Gauss-Legendre numerical integration method.

\section{Singular integrand}

In the case of the propagation of on-shell intermediate states, the polynomials of the denominators in Eqs. $(76,77$ ) acquire one (or two) roots in the domain of integration. Some simple physical considerations have shown that among the six diagrams numerically evaluated, the three processes where the photon in the $e p \rightarrow e p \gamma$ reaction is emitted from the initial electron line are free of poles (section IIIA). In contrast, the three vertex graphs where the photon is emitted from the final electron line were seen to contain singularities. The corresponding integrals are then defined by an analytical continuation into the complex plane and take the form :

$$
\int_{0}^{1} \frac{x_{1}^{m} d x_{1}}{\left(\alpha x_{1}+\beta \pm i \epsilon\right)^{n}} \quad \text { or } \quad \int_{0}^{1} \frac{x_{1}^{m} d x_{1}}{\left(\alpha x_{1}^{2}+\beta x_{1}+\gamma \pm i \epsilon\right)^{n}} .
$$

The prescription for on-shell propagation is of course already taken into account in the propagators and determines the sign in front of the $i \epsilon$ (which can also be obtained by applying the simple trick $m \rightarrow m-i \epsilon / 2$ ). Complications can occur from the possibility of two distinct roots in the interval $[0,1]$ for the second order polynomial. An important remark then concerns the variable of integration. In Eq. (75), the choice of $x_{1}$ as the more internal dimension was purely arbitrary. In fact all the decompositions in the three parameters have been derived and it has been shown that it was always possible to find an expansion providing at most one singularity.

In appendix B, we give the analytical results for the integrals of Eq. (78). We checked these results with a numerical method, where one pole along the interval $[0,1]$ is avoided by analytically continuing the integrand into the complex plane. In this way, the integral along $[0,1]$ is replaced by an integration along a semi-circle (with origin at $0.5+0 i$ and radius $1 / 2$ ) in the opposite complex half-plane with respect to the pole. A comparison between the two methods shows a perfect agreement. Only in the special cases where one pole comes close to an edge of the domain of integration $[0,1]$ (typically within a distance $m^{2} / E_{e}^{2}$ to 0 or 1 ), one has to increase the number of integration points of the numerical method to obtain the same precision. 


\section{Numerical checks and accuracy}

Thanks to the analytical calculation of the first integration in the Feynman parameter integrals under study, singularities on the real axis have been removed and the two remaining integrations can then be performed numerically using the Gauss-Legendre method. In the implementation of this algorithm the major difficulty consisted in finding the suitable binnig of the integration domain and to determine the number of points per bins. A detailed study of the integrated functions has been performed to estimate the width and amplitude of the sharp variations close to the ends of the domain. In this paragraph we discuss various checks of the precision of our results as well as their numerical stability.

A strong cross check of the reliability of our calculations is the exact agreement between two programs developed in paralell [29,30]. Both of them use the same numerical method but they have been coded independently using in most cases different decomposition of the terms and different order in the integration variables, which checks the symmetry in the permutation of $x_{1}, x_{2}$ and $x_{3}$ variables. Comparison at each intermediate stage of the calculation also excludes any missprints in the writing of the quite extensive expressions.

Besides this agreement between two independent programs, the next requirement is the numerical convergence of the calculations. Figs. 4 and 5 show results obtained for typical MAMI and JLab kinematics respectively. Beyond a certain density of integration bins and points per bin, the numerical instabilities are brought down to few $10^{-4}$ of the lowest order cross section. This accuracy is far below all the other theoretical uncertainties related to the performed approximations or experimental knowledge of the form factors (of the order of $1 \%$ ). Nevertheless this kind of very good convergence is useful since numerical instabilities can be amplified in the coherent sum of all the diagrams or when computing higher energy kinematics. In the case of the deeply virtual Compton scattering, we have checked that one has to double the number of integration points, to get the same numerical precision.

Some features of the electromagnetic interaction itself can also be used to check further the validity of our results. Let's consider the total amplitude of the sum of all the virtual radiative correction diagrams (Fig. 2). Denoting the Lorentz index associated with the real photon vertex by $\mu$, this amplitude can be written as the scalar product $T^{\mu} \epsilon_{\mu}^{*}$ where $\epsilon^{*}$ stands for the polarization vector of the real photon with four-vector $q^{\prime}$ and where $T^{\mu}$ represents the electromagnetic current. The gauge invariance of electromagnetism implies $T^{\mu} q_{\mu}^{\prime}=0$ and provides us with a powerful test of our calculations. Since our numerical accuracy is finite, we cannot get exactly zero. Therefore, we rather define a quantity compared to which the scalar product $T^{\mu} q_{\mu}^{\prime}$ has to be small. A natural quantity is the product of the norms of the two Lorentz vectors. The gauge invariance criteria thus becomes a test of the smallness of the following dimensionless ratio :

$$
\frac{\left|T^{\mu} q_{\mu}^{\prime}\right|^{2}}{\left|T^{\mu} T_{\mu}^{\dagger}\right|\left(q^{\prime 0}\right)^{2}} \ll 1
$$

This ratio is shown in Fig. 6 as a function of the angle between $q$ and $q^{\prime}$. The gauge invariance is verified by the fact that the smallest ratio (solid curve) stays in the range $\left[10^{-4}, 10^{-6}\right]$ and is obtained when the complete set of diagrams with analytical+numerical terms is included in $T^{\mu}$. 
As a last consistency check, we investigated the mass dependence of the virtual radiative corrections. The relative effect in the $\mathrm{BH}+$ Born cross section is illustrated in Fig. 7 for different values of the mass of the lepton. For this test we kept track of the mass dependence in all the kinematical variables. We observe that when increasing the lepton mass (at fixed lepton kinematics), the effect of the radiative corrections rapidly decreases, which reflects the suppression of photon emission by a heavy particle.

\section{Radiative corrections at the proton side and two-photon exchange corrections}

In section $[\mathrm{IIA}$ - IIIC, we calculated the radiative corrections to the $e p \rightarrow e p \gamma$ reaction, corresponding with the diagrams of Figs. 2 and 3 . They are the virtual radiative corrections at the lepton side, the vacuum polarization contributions and the soft-photon emission from the lepton. These can be calculated model-independently as has been shown above. Although these corrections are the dominant ones (when $Q^{2}>>m^{2}$, leading to large logarithms), we want to estimate in this section how large are the virtual radiative corrections at the proton side, the two-photon exchange corrections (direct and crossed box diagrams) and the soft-photon emission from the proton. Generally, the radiative corrections from the proton side are typically suppressed compared with those from the electron, due to the much larger mass of the proton. However, to calculate the first order radiative corrections to the $e p \rightarrow e p \gamma$ reaction which originate from the proton side, one needs a model for the VCS process. We do not aim in this paper, to calculate these corrections within a given model. However, to provide some quantitative estimate, we will follow the results of [24], where the corrections at the proton side were studied for elastic scattering.

The $Z$-dependent corrections originate from the interference between soft-photon emission from the electron and from the proton, and from the two-photon exchange contributions (direct and crossed box diagrams). Both processes contain IR divergences, which cancel in their sum at the cross section level. The interference between the soft-photon emission from the electron and from the proton can be calculated along the same lines as in appendix A5 for the electron (neglecting form factor effects in the soft-photon limit). For the two-photon exchange contributions, the calculation is dominated by those regions in the integration where one of the two exchanged photons is soft. Therefore, one can evaluate the rest of this amplitude by taking the momentum of either of the two exchanged photons to be zero. In this approximation, the original amplitude factorizes and one can follow the derivation of [24], where this same calculation has been performed for elastic scattering. Therefore, the $Z$-dependent radiative corrections can be estimated in the soft-photon limit by the same correction factor of Eq. A75) as for elastic scattering.

The $Z^{2}$-dependent corrections originate from the soft-bremsstrahlung from the proton and from the proton vertex corrections. In [24], these corrections have also been calculated for elastic scattering. For the soft-photon emission, one can again factorize the orginal amplitude, so that the same correction factor is obtained for the $e p \rightarrow e p \gamma$ reaction as for elastic scattering. The proton vertex correction has been split in 24 into two parts. The first part contains entirely the IR divergence, which cancels with the IR divergence from soft-photon emission from the proton, and in which the original amplitude factorizes. The second term in the proton vertex correction depends on the nucleon structure (form factor dependence for elastic scattering) and will be different when going from elastic scattering to 
the $e p \rightarrow e p \gamma$ reaction. For elastic scattering, this structure dependent term was however found [24] to be quite small, except when going to very large $Q^{2}$ (much larger than $M_{N}^{2}$ ). When staying in the few $\mathrm{GeV}^{2}$ region, this correction was calculated in [24] to be well below $1 \%$. Therefore, we approximate the $Z^{2}$ dependent correction to the $e p \rightarrow e p \gamma$ reaction by the structure-independent term of Eq. (A77), as calculated in [24], and will neglect in the following the structure dependent term.

\section{RADIATIVE TAIL FOR ELASTIC SCATTERING AND VCS}

Besides the knowledge of the virtual radiative corrections and the soft-photon emission contributions to the $e p \rightarrow e p \gamma$ reaction, which were studied in section [II], the accurate determination of the $e p \rightarrow e p \gamma$ cross section from measured spectra also implies the knowledge of the radiative tail. The radiative tail consists of the photon emission processes where a semi-hard photon (with energy not very small compared with e.g. the lepton energies) is radiated from the electron (or proton).

The radiative tail to elastic or inelastic lepton-nucleon scattering has been the subject of numerous studies in the literature [31,32,22, 33]. The elastic radiative tail also makes a sizeable contribution to the cross sections for deep-inelastic lepton-nucleon scattering (see e.g. [34]).

One should notice that the distinction between the soft-photon emission and the radiative tail is not a fundamental one, the latter being just the extension of photon emission processes to higher energies. Although the formulas given in this paper for the real radiative corrections can in principle be extended and applied to higher energies (e.g. Eqs. (A71A73) for elastic scattering), in some cases the characteristics of the experimental detection apparatus can be such that the cut in $E_{e}^{\prime e l}-E_{e}^{\prime}$ (elastic case) or in the missing mass $M_{x}^{2}$ $\left(M_{m 1}^{2}\right.$ or $M_{m 2}^{2}$ for the VCS case) cannot be cleanly defined, because the apparatus can have a changing acceptance as a function of $E_{e}^{\prime e l}-E_{e}^{\prime}$ or $M_{x}^{2}$, introducing a bias in the radiative tail. Therefore, it is useful to consider the radiative tail separately and to generate it in a Monte Carlo simulation. In doing such a simulation, it can be very helpful to have a "recipe", because it is a way to fold radiative effects with acceptance functions and other effects (e.g. multiple scattering, energy loss by collision, external radiative effects). In the literature such "recipes" were quite often presented. Many of them are based on one or another version of the peaking approximation, introduced originally by Schiff [35]. In the peaking approximation, the photon is radiated along either the initial or final electron directions, i.e. the direction of the electron is not changed while radiating, only its energy is changed.

Below, we start by giving such a recipe, based on the formulas presented in this paper. What one essentially needs for a Monte Carlo simulation is an electron energy loss distribution due to real internal radiative effects. For each event one can then sample in such a distribution, both for the incoming and the outgoing electron. We next give a comparison between such a method based on the peaking approximation, with an exact numerical calculation of the radiative tail. We show to what extent the full calculation validates the approximate method for the case of elastic electron-nucleon scattering, and show that this method is realistic enough to apply it next to the calculation of the radiative tail in the case of the VCS. 


\section{A. Energy loss distribution for real internal radiative effects}

The details of the calculation of the real radiative corrections can be found in appendix A. It is discussed there how the real internal radiative corrections give rise to a correction factor $e^{\delta_{R}}$ to the cross section. The part of $\delta_{R}$ giving rise to the radiative tail (when differentiating $\delta_{R}$ with respect to the electron energy loss) is the first term of Eq. (A66), which contains the maximal energy of the emitted photon $\Delta E_{s}$, which is defined as in Eq. (A49). The correction factor $e^{\delta_{R}}$ can be written as the product of a number of factors, of which the first one is given by :

$$
\left(\frac{\left(\Delta E_{s}\right)^{2}}{\tilde{E}_{e} \tilde{E}_{e}^{\prime}}\right)^{a}
$$

where $a$ is given by (see Eq. (A66)) :

$$
a=\frac{\alpha_{e m}}{\pi}\left[\ln \left(\frac{Q^{2}}{m^{2}}\right)-1\right]
$$

and where the tilded quantities in Eq. (80) are expressed in the c.m. system of (soft photon + recoiling proton) as explained in appendix A5. Because in a simulation it is more straightforward to apply radiative effects in the lab, we express Eq. (80) in lab quantities, by using Eq. (A49), which yields :

$$
\left(\frac{\left(\eta \Delta E_{e}^{\prime}\right)^{2}}{E_{e} E_{e}^{\prime}}\right)^{a}
$$

where $\Delta E_{e}^{\prime}=E_{e}^{\prime e l}-E_{e}^{\prime}$. Introducing furthermore the quantity $\Delta E_{e}=\eta^{2} \Delta E_{e}^{\prime}$, we can write Eq. (82) as :

$$
\left(\frac{\left(\eta \Delta E_{e}^{\prime}\right)^{2}}{E_{e} E_{e}^{\prime}}\right)^{a}=\left(\frac{\Delta E_{e} \Delta E_{e}^{\prime}}{E_{e} E_{e}^{\prime}}\right)^{a}=\left(\frac{\Delta E_{e}}{E_{e}}\right)^{a}\left(\frac{\Delta E_{e}^{\prime}}{E_{e}^{\prime}}\right)^{a}
$$

The energy changes $\Delta E_{e}\left(\Delta E_{e}^{\prime}\right)$ can be interpreted as the energy losses of the incoming (outgoing) electron due to radiation before (after) the scattering process respectively. We can then interpret the factor $\left(\frac{\Delta E_{e}}{E_{e}}\right)^{a}$ as the fraction of incoming electrons which have lost an energy between 0 and $\Delta E_{e}$, after being subject to real internal radiation in an equivalent radiator with thickness $a$. The factor $\left(\frac{\Delta E_{e}^{\prime}}{E_{e}^{\prime}}\right)^{a}$ has a similar interpretation, but then on the outgoing electron side 円. Given this interpretation, if one uses a $\Delta E$ distribution $I_{\text {int }}(E, \Delta E, a)$, which satisfies :

\footnotetext{
${ }^{1}$ Note that when applying Eq. (83) to the radiative tail, i.e. when considering the emission of a photon whose energy is not very small compared with the electron energies, we calculate $E_{e}^{\prime e l}$ in the formula for $\Delta E_{e}^{\prime}$, using the elastic scattered energy corresponding with an initial electron which has radiated and whose energy is given by $E_{e}-\Delta E_{e}$. In the soft-photon limit this difference disappears.
} 


$$
\int_{0}^{\Delta E} I_{\text {int }}(E, \Delta E, a) d(\Delta E)=\left(\frac{\Delta E}{E}\right)^{a}
$$

then it is clear that by sampling such a distribution in a Monte Carlo simulation, the correction factor is correctly obtained. The distribution $I_{\text {int }}$, which has this property is given by :

$$
I_{\text {int }}(E, \Delta E, a)=\frac{a}{\Delta E}\left(\frac{\Delta E}{E}\right)^{a}
$$

and is normalized to 1 :

$$
\int_{0}^{E} I_{\text {int }}(E, \Delta E, a) d(\Delta E)=1
$$

\section{B. Evaluation of the radiative tail and comparison with an exact numerical calculation for elastic electron-proton scattering}

Given the above distribution, a method for introducing a radiative tail due to internal radiation in a Monte Carlo simulation for elastic electron scattering suggests itself :

i) For the incoming electron, sample an energy loss $\Delta E_{e}$ using the distribution (85) with $E=E_{e}$ the incoming electron energy.

ii) Apply elastic electron scattering using the reduced electron energy $E_{e}-\Delta E_{e}$, and if the cross section behavior is implemented in the simulation, use the elastic scattering cross section at the reduced electron energy. After the elastic scattering process, the outgoing electron has an energy $E_{e}^{\prime e l}$.

iii) For the outgoing electron, sample an energy loss $\Delta E_{e}^{\prime}$ using the distribution (85) with $E=E_{e}^{\prime e l}$. The final electron energy is now $E_{e}^{\prime e l}-\Delta E_{e}^{\prime}$.

To calculate the equivalent radiator thickness $a$ of Eq. (81), one needs the value of $Q^{2}$, which one can in principle only calculate after the complete process has taken place. However, one can show that the above procedure reproduces the correction factor (82) with a very good accuracy already by calculating the value of $Q^{2}$ with elastic electron scattering kinematics.

It is intuitively clear that the above procedure, in the case where a constant cross section is used, will reproduce the correction factor of Eq. (82). In case the actual elastic scattering cross section behavior is implemented, the cross section "walk" with the incoming electron energy is taken into account. Remark that the above procedure implies an electron energy loss both at the incoming and the outgoing electron sides.

The discussed method implies, however, the assumption of a strict alignment of the bremsstrahlung photons in the direction of the radiating leptons, which is known as the (angular) peaking approximation. The strength on the other hand is found by integrating the correct angular shape in the soft photon limit, as done in appendix A5. To test the validity of this approximate procedure, we performed a fully numerical calculation of the radiative tail for elastic electron-proton scattering. It consists of integrating over the photon phase space in the diagrams where a photon is emitted from an electron (cfr. BH diagrams of Fig. 1 (a) and (b)), as well as the diagrams where a photon is emitted from the nucleon (cfr. Born 
diagrams of Fig. 1 (c) and (d)). In doing so, we nowhere neglect the photon momentum $l$, in contrast to the calculation of appendix A5 in the soft-photon limit, where this momentum is neglected with respect to the lepton momenta. For fixed electron kinematics, the angular phase space of the soft photon is covered by a grid with about 225000 points, chosen with increased density in the peak regions in order to keep the point-to-point change of the cross section smaller than $10 \%$. Attention has to be paid right in the middle of the peaks where the cross section drops very rapidly to (practically) zero within the characteristic angle $m / E_{e}$, as shown in Fig. 8. More details on this numerical integration can be found in [36. The result of this integration is the absolute cross section of the radiative tail, differential in the outgoing electron's momentum and angles. It is shown by the points in Fig. 9 for $E_{e}=855.0 \mathrm{MeV}$ and $\theta_{e}=52.18^{\circ}$. The energy of the outgoing electron is then determined by $E_{e}^{\prime}=E_{e}^{\prime e l}-\Delta E_{e}^{\prime}$. The points are compared with the analytical result in the soft-photon limit, obtained by differentiating the expression of Eq. (A66) for $\delta_{R}$ - for photon emission from the electron - with respect to $\Delta E_{e}^{\prime}$. This gives a strict $\Delta E_{e}^{\prime-1}$ behaviour, yielding the cross section $\sigma_{a} \equiv \sigma_{\text {Born }} a / \Delta E_{e}^{\prime}$ where the proportionality factor $a$ is given as in Eq. (81). The soft-photon formula gives thus a straight line when both the cross section and $\Delta E_{e}^{\prime}$ are presented on a logarithmic scale. The deviation can be seen in the lower plot of Fig. 9. From the keV-region up to about $1 \mathrm{MeV}$ for $\Delta E_{e}^{\prime}$, the deviation is less then $10^{-3}$ which can be taken as an upper limit for the error of the numerical integration procedure. This agreement demonstrates that the soft-photon approximation holds to very good precision in this region. For higher values of $\Delta E_{e}^{\prime}$, a raise of the photon emission cross section is observed as is expected due to the change of kinematics leading to a lower momentum transfer to the proton, and to a resulting "walk" of the cross section. We also show on the lower plot of Fig. 9 the result when both radiation from the electron and proton are considered. For better presentation, both results are normalized to the cross section $\sigma_{a}$ for soft-photon emission from the electron, as defined above.

In Fig. 10, we compare for two kinematics the exact numerical calculation of the radiative tail with the approximate method of the Monte Carlo simulation as discussed above. The simulation has been investigated by running it with and without the cross section behaviour (dipole form factors assumed), and the ratio between the two versions is presented by the lines, the outer lines representing the statistcal accuracy. One notices that the increase of the radiative tail is reproduced, but somewhat overestimated compared with the exact calculation.

\section{Application to virtual Compton scattering}

The above procedure can also be applied to VCS, as long as the angular peaking approximation is used, i.e. the electron does not change its direction while losing energy by internal real radiation. Indeed, Eq. (58) is completely similar to the elastic case, when expressing it in the c.m. system of either (soft photon + outgoing nucleon) or (soft-photon + outgoing photon) depending on how the $e p \rightarrow e p \gamma$ reaction is measured, as explained in section [IIB. After exponentiation, one can apply a factorization completely similar as in Eq. (83). Because under the assumption of the angular peaking approximation $\Delta E_{s} / \tilde{E}$ is constant under a Lorentz transformation, we obtain the property that the shape of the distribution (85) is system independent, only its endpoint value $E$ changes. As a result, 
one can apply the distribution of Eq. (85) in the lab for VCS, but then using lab values for $E_{e}$ and $E_{e}^{\prime}$. For VCS, one certainly can have a changing acceptance of the detection apparatus as a function of missing mass (making a "clean" cut in missing mass on the data impossible), so that generating a radiative tail in a Monte Carlo simulation with the above described method is probably the best way to implement the radiative tail correction to the data. Such a simulation was implemented for the VCS experiments already performed at MAMI [11] and at JLab [12], and will be fully described in a forthcoming paper [37].

\section{RESULTS AND DISCUSSION}

\section{A. Elastic electron-proton scattering}

Before showing results for VCS, we briefly discuss first the effect of the radiative corrections to elastic electron-proton scattering, in order to have a point of reference. The radiative corrections to elastic electron-proton scattering are presented in detail in appendix A. In Table 目, we show for different elastic kinematics (MAMI, JLab) the numerical values of the vertex correction $\left(\delta_{\text {vertex }}\right.$ of Eq. (A68) $)$, the vacuum polarization correction $\left(\delta_{v a c}\right.$ of Eq. (A70) ), and the real radiative correction at the electron side $\left(\delta_{R}\right.$ of Eq. (A66) $)$. We also

show the $Z$ and $Z^{2}$ dependent corrections, $\delta_{1}$ (Eq. (A75) ) and $\delta_{2}^{(0)}$ (Eq. (A77)) respectively, as derived in the recent work of [24]. We omit here the small part in the $Z^{2}$ dependent correction which depends on the particular model for the nucleon structure (in the elastic case, the form factors), as can be found in [24]. In Table [], we indicate the total radiative correction $\delta_{\text {tot }}$ as the sum of all the different contributions as in Eq. (A74). From Table [1, we see that by far the largest contribution to the radiative correction comes from the large logarithm and double logarithm in $Q^{2} / m^{2}$ in the electron vertex correction. When evaluating the real radiative corrections for $E_{e}^{\prime e l}-E_{e}^{\prime}=0.01 E_{e}$, the total effect of the radiative correction is an upwards correction of the data (for negative $\delta_{t o t}$ ) of the order $20-25 \%$. In the last column of Table 1 (denoted by EXP), we also indicate the result when exponentiating all corrections except the vacuum polarization contribution, which - as modification of the photon propagator - is resummed as in Eq. (A73). One sees that this can lead to differences of the order of $2 \%$.

In an elastic scattering experiment, one measures an scattered electron spectrum and has to evaluate the real radiative corrections as a function of the cut $\left(E_{e}^{\prime e l}-E_{e}^{\prime}\right)$ which one performs in the spectrum. Dividing the measured cross section by the correction factor $\left(1+\delta_{t o t}\right)$ and plotting the result as function of $\left(E_{e}^{\prime e l}-E_{e}^{\prime}\right)$, should then lead to a "plateau" behavior, which demonstrates the consistency of the procedure (within a certain range of the value $\left(E_{e}^{\prime e l}-E_{e}^{\prime}\right)$ where one knows the radiative tail to sufficient accuracy).

The determination of the elastic cross section for the kinematics $E_{e}=705.11 \mathrm{MeV}, \theta_{e}$ $=42.6^{\circ}$ is shown in Fig. 12. The upper plot shows the dE-spectrum of elastic data taken (during the beam time of the VCS experiment) at MAMI. It is compared with the simulated spectrum (dashed line). On the lower plot, the ratio of the experimental spectrum integrated up to the value $\Delta E_{e}^{\prime}$, to the simulation integrated also up to $\Delta E_{e}^{\prime}$ is shown as function of the cutoff energy $\Delta E_{e}^{\prime}$. This gives the elastic cross section, which is seen to be stable below the $1 \%$-level over a long interval up to the cut by the acceptance of the spectrometer. The slow descent for higher $\Delta E_{e}^{\prime}$ indicates that the simulation overestimates slightly the radiative tail. 


\section{B. VCS below pion production threshold}

We next turn to the $e p \rightarrow e p \gamma$ reaction below pion threshold. It was discussed in section [II, that the lowest order (in $\alpha_{e m}$ ) amplitude of the $e p \rightarrow e p \gamma$ process at low outgoing photon energies $\mathrm{q}^{\prime} \equiv\left|\vec{q}^{\prime}\right|$ is given by the $\mathrm{BH}+$ Born processes. The deviation from the $\mathrm{BH}+$ Born amplitudes grows with $\mathrm{q}^{\prime}$, and can be parametrized (at low $\mathrm{q}^{\prime}$ ) in terms of six generalized polarizabilities (GP's) of the nucleon, which are function of $Q^{2}$.

A first VCS experiment has been performed at MAMI [11]. It consisted of measuring the $e p \rightarrow e p \gamma$ reaction at five values of $\mathrm{q}^{\prime}$ below pion threshold, ranging from $\mathrm{q}^{\prime}=33 \mathrm{MeV} / \mathrm{c}$ to $\mathrm{q}^{\prime}=111.5 \mathrm{MeV} / \mathrm{c}$. At the lowest value $\mathrm{q}^{\prime}=33 \mathrm{MeV} / \mathrm{c}$, where the polarizability effect is negligeably small, the measurement serves as a check of the Low Energy Theorem (LET). The measured deviation as function of $\mathrm{q}^{\prime}$ can then be interpreted as the effect of the GP's. It is clear that both to test the LET as well as to extract the GP's from the measured deviation with respect to the $\mathrm{BH}+$ Born result (which is expected to be of the order 10 $20 \%$ at the highest $\mathrm{q}^{\prime}$ value), it is a prerequisite to know very accurately how the result is modified due to radiative corrections.

In Fig. 13, we first show the differential cross section for MAMI kinematics at a low value $\mathrm{q}^{\prime}=33 \mathrm{MeV} / \mathrm{c}$, as function of the c.m. angle of the emitted real photon with respect to the direction of the virtual photon. One sees from Fig. 13 that the virtual radiative corrections reduce the $\mathrm{BH}+$ Born result in these kinematics by about $16 \%$ (or when applied to data, increase the uncorrected data by $16 \%$ ). The real radiative corrections have to be estimated as function of the cut which one performs in the missing mass spectrum. The VCS experiments below pion threshold measure the $e p \rightarrow e p \gamma$ reaction by detecting the outgoing electron and proton, and reconstruct the missing mass $M_{m 2}$ as defined in Eq. (64). In Fig. 13, the real radiative corrections are shown for a value of $\Delta E_{s}=10 \mathrm{MeV}$, where the soft-photon energy $\Delta E_{s}$ is determined from the cut in the missing mass according to Eq. (68). For the small value $\mathrm{q}^{\prime}=33 \mathrm{MeV} / \mathrm{c}$, the real radiative correction depends only very little on the angle $\theta_{\gamma \gamma}$ (through the last terms on the rhs of Eqs. (65.,66)). For $\Delta E_{s}$ $=10 \mathrm{MeV}$, the real radiative correction $\delta_{R}$ is given by $\delta_{R} \approx-0.025$, which corresponds with increasing the uncorrected data by about $2.5 \%$. For $\Delta E_{s}=20 \mathrm{MeV}, \delta_{R} \approx+0.02$ (reducing the uncorrected data by about $2 \%$ ), and for $\Delta E_{s}=30 \mathrm{MeV}, \delta_{R} \approx+0.045$ (reducing the uncorrected data by about $4.5 \%$ ). To determine the $e p \rightarrow e p \gamma$ cross section from the measured missing mass spectra, one has to perform a consistency check by plotting the experimentally measured (uncorrected) cross section divided by the radiative correction factor as function of the cut in the missing mass spectrum. In this way, one has to find a "plateau" behavior, as was demonstrated before for elastic data. This consistency check was also performed on the VCS data measured at MAMI [11], and will be shown in a forthcoming publication [37].

In Fig. 14, we show the the differential cross section for MAMI kinematics at the highest measured outgoing photon energy : $\mathrm{q}^{\prime}=111.5 \mathrm{MeV} / \mathrm{c}$. The virtual radiative corrections are mainly $\mathrm{q}^{\prime}$ independent (for these rather small values) and lead thus also here to a reduction of the $\mathrm{BH}+$ Born result by about $16 \%$. The real radiative corrections are again shown for $\Delta E_{s}=10 \mathrm{MeV}$, and exhibit a slight angular dependence. These corrections were applied

to the data from the unpolarized MAMI experiment of [11]. From the deviation of the radiatively corrected data and the $\mathrm{BH}+$ Born result, two combinations of GP's have been 
extracted at $Q^{2} \simeq 0.33 \mathrm{GeV}^{2}$ in [1].

An experiment below pion production threshold to measure the GP's at higher $Q^{2}$ has also been measured at JLab [12] and is under analysis at the time of writing. In Fig. 15, we show how the $\mathrm{BH}+$ Born cross section is modified due to the virtual radiative corrections. It is seen that for the JLab kinematics of Fig. 15, the $\mathrm{BH}+$ Born result is reduced at the backward angles by about $20 \%$ due to the virtual radiative corrections.

The unpolarized VCS cross section below pion threshold provides three independent structure functions (when varying the value of $\varepsilon$ in the experiment), which allows to extract three of the six (lowest order) generalized nucleon polarizabilities. To extract the three remaining nucleon polarizabilities, one has to resort to double polarization observables as discussed in [38]. In particular, double polarization observables with polarized electron beam and with a polarized target (along either of the three axes), or alternatively by measuring the recoil nucleon polarization, provide three new observables to extract the three additional nucleon response functions [38,2]. In Fig. 16, we show the double polarization asymmetries for MAMI kinematics, by measuring the recoil polarization components along the $z$-direction (virtual photon direction) or along the $x$-direction (perpendicular to the virtual photon but parallel to the scattering plane). One aims to extract the polarizability effect in these observables from the deviation of the measured asymmetry and the BH + Born result (see e.g. 38] for an estimate of this effect within a model calculation). Therefore, it is important to know how much the $\mathrm{BH}+$ Born result is affected by the radiative corrections before extracting the polarizability effect. It is seen in Fig. 16 that the effect of the radiative corrections on the double polarization asymmetries nearly drops out in the ratio (much less than $1 \%$ change of the asymmetries). At the low values of the outgoing photon energy $\mathrm{q}^{\prime}$ (e.g. $\mathrm{q}^{\prime} \simeq 33 \mathrm{MeV} / \mathrm{c}$ ) where the polarizability effect is very small, these asymmetries are also hardly affected by radiative corrections. Therefore, these asymmetries can also provide an independent check of the LET. An experiment to measure the VCS double polarization observables by measuring the recoil nucleon polarization is planned at MAMI in the near future [39.

\section{Deeply virtual Compton scattering}

Besides the low energy region, the VCS process is also studied in the Bjorken regime, where $Q^{2}$ and $\nu=p . q / M_{N}$ are large, with $x_{B}=Q^{2} /\left(2 M_{N} \nu\right)$ fixed. In this kinematical region, the process is refered to as deeply virtual Compton scattering (DVCS). In the Bjorken regime, the DVCS amplitude factorizes into a perturbatively calculable hard scattering amplitude, and into a non-perturbative part at the proton side, expressed in terms of so-called skewed parton distributions (SPD's) which generalize the ordinary parton distributions. These SPD's are new nucleon structure observables which one aims to extract by measuring e.g. the exclusive $e p \rightarrow e p \gamma$ reaction in the Bjorken regime. Similarly as was seen before in the threshold region, the $e p \rightarrow e p \gamma$ reaction can have an important contribution from the $\mathrm{BH}$ process, besides the DVCS process of actual interest. However, the BH and DVCS contributions behave differently as function of the lepton beam energy, as studied in Refs. 40,2, 21]. In particular, at the lower beam energies, such as e.g. available at JLab, the BH process dominates in the forward direction over the DVCS process. In this region, the DVCS

process becomes only measurable due to its interference with the $\mathrm{BH}$ process. In order to 
extract the DVCS process (and the nucleon structure information) from its interference with the $\mathrm{BH}$, it is therefore important to have good knowledge of how the radiative corrections modify the $\mathrm{BH}$ amplitude.

In Fig. 17, we show the $e p \rightarrow e p \gamma$ cross section in kinematics accessible at JLab, where such an experiment is planned [17]. The DVCS cross section is calculated by using the ansatz for the SPD's of [11]. It is seen from Fig. 17, that the BH indeed dominates over the DVCS cross section in these kinematics, and that the DVCS cross section gets enhanced due to its interference with the $\mathrm{BH}$. One furthermore sees that the virtual radiative corrections reduce the $\mathrm{BH}+$ DVCS cross section by about $23 \%$ in these kinematics. This is mainly due to the reduction of the $\mathrm{BH}$ process when including virtual radiative corrections. The real radiative corrections are shown in Fig. 17 for a value $\Delta E_{s}=0.1 \mathrm{GeV}$, which corresponds with a cut in the recoiling hadronic missing mass spectrum (defined in Eq. (59)) of $M_{m 1}^{2}$ $M_{N}^{2} \simeq 0.21 \mathrm{GeV}^{2}$.

In Ref. [2], it was suggested that an exploratory study of the DVCS process might be possible by studying the $e p \rightarrow e p \gamma$ reaction with a polarized electron beam. The electron single spin asymmetry (SSA) does not vanish out of plane and is only due to the interference of the $\mathrm{BH}$ amplitude and the imaginary part of the DVCS amplitude (i.e. the $\mathrm{BH}$ amplitude does not lead to a SSA, because it is purely real). Therefore, one expects this SSA to be less sensitive to radiative corrections on the $\mathrm{BH}$ amplitude. However, as the $\mathrm{BH}$ amplitude enters the SSA linearly in the numerator, but quadratically in the denominator (as in the unpolarized cross section), one might wonder what is the residual effect of the radiative corrections on this observable. In Fig. 18, we show the SSA for DVCS at JLab. One sees that the SSA gets only slightly reduced due to the radiative corrections. The reduction of the SSA amounts to maximum $5 \%$ of its value around $5^{\circ}$, where the asymmetry reaches its maximal value. Therefore, the SSA shows to be a rather "clean" observable for extracting the DVCS amplitude in a region where the BH process dominates. Its measurement is also envisaged at JLab in the near future [17].

\section{CONCLUSIONS}

We studied in this work the first order QED radiative corrections to the ep $\rightarrow$ ep $\gamma$ reaction. The one-loop virtual radiative corrections have been evaluated by a combined analytical-numerical method. Several tests were shown to cross-check the numerical method used. Furthermore, it was shown how all IR divergences cancel when adding the soft-photon emission processes. A fully numerical method was presented for the photon emission processes where the photon energy is not very small compared with the electron energies, which makes up the radiative tail. Besides, we have also presented an approximate calculation of the radiative tail, which was shown to be realistic enough for use in a Monte Carlo simulation.

We compared our results first to elastic electron-proton scattering. Subsequently, the results for the radiative corrections to the $e p \rightarrow e p \gamma$ reaction were shown both below pion threshold and in the deeply virtual Compton scattering regime.

Below pion threshold, our calculations were applied to the first dedicated VCS experiment at MAMI, and show that the effect of the radiative corrections results in an enhancement of

the uncorrected data by about $20 \%$ (or an equivalent reduction of the theory). VCS double 
polarization asymmetries where shown to be insensitive to radiative corrections.

For the DVCS, we calculated radiative corrections for JLab kinematics and found the virtual radiative corrections to lead to an enhancement of the data by about $23 \%$. The single spin asymmetry was shown to be only slightly reduced by radiative corrections.

Although we focussed here on the kinematical regimes of ongoing or planned experiments, the present work can also serve as a tool in the analysis of future VCS experiments.

\section{ACKNOWLEDGMENTS}

As this work grew over a period of a couple of years, we enjoyed discussions with many colleagues about the subject of this work, in particular with N. d'Hose and P. Vernin, about the application of radiative corrections to elastic and VCS data. We thank M. Distler and H. Merkel for their help in the numerical implementation of the radiative tail calculation. Furthermore, we would like to thank P.A.M. Guichon for many discussions, which were at the origin of this work. We also want to acknowledge in particular very useful discussions and correspondence with L. Maximon and are grateful for his continued interest in this work.

This work was supported in part by the French Commissariat à l'Energie Atomique (CEA), by the EU/TMR contract ERB FMRX-CT96-0008, by the Deutsche Forschungsge-

meinschaft (SFB 443), by the French CNRS/IN2P3, and by the Fund for Scientific Research - Flanders (Belgium). 


\section{APPENDIX A: RADIATIVE CORRECTIONS TO ELASTIC LEPTON-NUCLEON SCATTERING USING THE DIMENSIONAL REGULARIZATION METHOD FOR BOTH UV AND IR DIVERGENCES.}

In this appendix, we provide the reader with some details of the derivation of the radiative corrections to elastic lepton scattering at one-loop level. In our derivation, we use the dimensional regularization procedure to regularize both ultraviolet and infrared divergences.

After a short introduction of the renormalization method, we calculate subsequently the vertex diagram at the lepton side (Fig. 19(a)), the lepton self-energy diagram (Fig. 19(b)), the vacuum polarization diagram (Fig. 19(c)), and give an analytical result, without approximations, for the soft photon emission at the lepton side (Fig. 19 (d) and (e)). We compare our results with other derivations found in the literature. At the end we collect the results to correct the elastic lepton-nucleon scattering cross sections and discuss the role of the radiative corrections at the proton side and the two-photon exchange corrections by referring to the recent work of Ref. [24]. In this appendix, we use the same notations as explained in section 凹.

\section{Renormalization method}

In calculating QED radiative corrections in this work, we are using the BPHZ renormalization method (as explained e.g. in Ref. [42]), which consists of replacing in the unrenormalized Lagrangian all bare quantities by renormalized ones. For QED, the bare Lagrangian is given by (we are using the conventions of Bjorken and Drell 25] in this work)

$$
\mathcal{L}_{\mathcal{B}}=\bar{\Psi}_{B}\left(i \gamma^{\mu} \partial_{\mu}-m_{B}\right) \Psi_{B}-\frac{1}{4} F_{B \mu \nu} F_{B}{ }^{\mu \nu}-e_{B} \bar{\Psi}_{B} \gamma^{\mu} \Psi_{B} A_{B \mu}
$$

where the bare field tensor $F_{B}^{\mu \nu}$ is given by

$$
F_{B}^{\mu \nu}=\partial^{\mu} A_{B}^{\nu}-\partial^{\nu} A_{B}^{\mu}
$$

The renormalization of the theory amounts in redefining the bare quantities in terms of renormalized (i.e. physical observable) ones :

$$
\begin{array}{ll}
\Psi_{B}=Z_{2}^{1 / 2} \Psi, & A_{B}{ }^{\mu}=Z_{3}^{1 / 2} A^{\mu}, \\
m_{B}=Z_{m} m, & e_{B}=Z_{g} e .
\end{array}
$$

In Eq. (A3), the renormalized finite quantities are $\Psi, A^{\mu}, m$ and $e$. A theory in which all divergences can be absorbed into renormalization constants such as $Z_{2}, Z_{3}, Z_{m}$ and $Z_{g}$ in Eq. (A3), is called multiplicatively renormalizable. This procedure leads to a decomposition of the QED Lagrangian of Eq. (A1) into

$$
\mathcal{L}_{\mathcal{B}}=\mathcal{L}_{\mathcal{R}}+\mathcal{L}_{\mathcal{C} T}
$$

where $\mathcal{L}_{\mathcal{R}}$ represents the renormalized Lagrangian in terms of the physical (finite) quantities

$$
\mathcal{L}_{\mathcal{R}}=\bar{\Psi}\left(i \gamma^{\mu} \partial_{\mu}-m\right) \Psi-\frac{1}{4} F_{\mu \nu} F^{\mu \nu}-e \bar{\Psi} \gamma^{\mu} \Psi A_{\mu}
$$


and where $\mathcal{L}_{\mathcal{C T}}$ is called the counterterm Lagrangian

$\mathcal{L}_{\mathcal{C} \mathcal{T}}=\left(Z_{2}-1\right) \bar{\Psi} i \gamma^{\mu} \partial_{\mu} \Psi-\left(Z_{2} Z_{m}-1\right) \bar{\Psi} m \Psi-\left(Z_{3}-1\right) \frac{1}{4} F_{\mu \nu} F^{\mu \nu}-\left(Z_{1}-1\right) e \bar{\Psi} \gamma^{\mu} \Psi A_{\mu}$

In Eq. (A6), the vertex renormalization constant $Z_{1}$ is defined as $Z_{1}=Z_{g} Z_{2} Z_{3}^{1 / 2}$. For a renormalizable theory such as QED, all divergences obtained by calculating loop diagrams with the renormalized Lagrangian $\mathcal{L}_{\mathcal{R}}$ are cancelled by the corresponding contributions in the counterterm Lagrangian $\mathcal{L}_{\mathcal{C T}}$. It will be shown below how the QED renormalization constants are calculated to order $O\left(e^{2}\right)$ by calculating the vertex diagram, the lepton selfenergy diagram and the photon polarization diagram at the one-loop level.

As QED is a gauge invariant theory, we will simplify all calculations in this work by using the Feynman gauge.

\section{Vertex diagram}

The on-shell photon-lepton-lepton vertex is represented by

$$
M_{v}^{\mu}=\bar{u}\left(k^{\prime}, h^{\prime}\right)\left[-i e \Lambda^{\mu}\left(k^{\prime}, k\right)\right] u(k, h),
$$

and the on-shell vertex of Eq. (A7) can be parametrized as

$$
\bar{u}\left(k^{\prime}, h^{\prime}\right) \Lambda^{\mu}\left(k^{\prime}, k\right) u(k, h)=\bar{u}\left(k^{\prime}, h^{\prime}\right)\left[\left(1+F\left(Q^{2}\right)\right) \gamma^{\mu}-G\left(Q^{2}\right) i \sigma^{\mu \nu} \frac{q_{\nu}}{2 m}\right] u(k, h),
$$

where $q=k-k^{\prime}$.

To order $O\left(e^{2}\right)$, the vertex $\Lambda^{\mu}$ (corresponding with Fig. 19(a)) is given by

$$
\Lambda^{\mu}\left(k^{\prime}, k\right)=\gamma^{\mu}-i e^{2} \mu^{4-D} \int \frac{d^{D} l}{(2 \pi)^{D}} \frac{\gamma^{\alpha}\left(\not k^{\prime}+\not l+m\right) \gamma^{\mu}(\not k+\not l+m) \gamma_{\alpha}}{\left[l^{2}\right]\left[l^{2}+2 l . k^{\prime}\right]\left[l^{2}+2 l . k\right]}+O\left(e^{4}\right),
$$

where a mass scale $\mu$ (renormalization scale) has to be introduced when passing to $D \neq 4$ dimensions in order to keep the coupling constant dimensionless. It is immediately seen by power counting that in four dimensions $(D=4)$, the one-loop integral in Eq. (A9) contains an ultraviolet $(l \rightarrow \infty)$ logarithmic divergence and an infrared $(l \rightarrow 0)$ logarithmic divergence. To subtract the divergent parts (by the corresponding counterterms) of expressions such as Eq. (A9), one has to regularize them first.

We follow in this work the dimensional regularization procedure to regularize both ultraviolet and infrared divergences. The dimensional regularization method amounts in calculating loop diagrams in $D$ dimensions. Physical observables are obtained by letting $D \rightarrow 4$ at the end. To obtain an integral which is ultraviolet convergent, one has to take $D<4$, or $\epsilon_{U V} \equiv 2-D / 2>0$ in expressions such as Eq. (A9). To obtain an integral which is infrared convergent, one has to take $D>4$, or $\epsilon_{I R} \equiv 2-D / 2<0$. The two different limits show that care has to be taken with the limit $D \rightarrow 4$, which means that the parts in Eq. (A9) that are 
infrared divergent and the parts that are ultraviolet divergent have to be separated and in the corresponding terms, two different limits have to be taken when one approaches $D=4$. Although the dimensional regularization scheme has been applied originally to ultraviolet divergent expressions as it respects the symmetries of the theory (in particular the gauge symmetry for a gauge theory), it has also been applied in a few works to regularize infrared divergences 43,44.

When working out the integral in Eq. (A9), one obtains after some algebra the following expressions for $F\left(Q^{2}\right)$ and $G\left(Q^{2}\right)$ to order $O\left(e^{2}\right)$ :

$$
\begin{aligned}
F\left(Q^{2}\right)=\frac{e^{2}}{(4 \pi)^{2}} & \left\{\left[\frac{1}{\varepsilon_{U V}}-\gamma_{E}+\ln \left(\frac{4 \pi \mu^{2}}{m^{2}}\right)\right]+\left[\frac{1}{\varepsilon_{I R}}-\gamma_{E}+\ln \left(\frac{4 \pi \mu^{2}}{m^{2}}\right)\right] \frac{v^{2}+1}{v} \ln \left(\frac{v+1}{v-1}\right)\right. \\
& +\frac{v^{2}+1}{2 v} \ln \left(\frac{v+1}{v-1}\right) \ln \left(\frac{v^{2}-1}{4 v^{2}}\right)+\frac{2 v^{2}+1}{v} \ln \left(\frac{v+1}{v-1}\right) \\
& \left.+\frac{v^{2}+1}{v}\left[S p\left(\frac{v+1}{2 v}\right)-S p\left(\frac{v-1}{2 v}\right)\right]\right\},
\end{aligned}
$$

and

$$
G\left(Q^{2}\right)=\frac{e^{2}}{(4 \pi)^{2}} \frac{v^{2}-1}{v} \ln \left(\frac{v+1}{v-1}\right)
$$

where $v$ is given by

$$
v^{2} \equiv 1+\frac{4 m^{2}}{Q^{2}}
$$

with $Q^{2}=-q^{2}>0$. In Eq. (A10), $\gamma_{E}$ represents the Euler constant, and the Spence (or dilogarithmic) function is defined by

$$
S p(x) \equiv-\int_{0}^{x} d t \frac{\ln (1-t)}{t} .
$$

From Eq. (A11), the one-loop radiative correction to the electron magnetic moment follows as

$$
\mu=\frac{e}{2 m}\left(1+G\left(Q^{2}=0\right)\right)=\frac{e}{2 m}\left(1+\frac{\alpha_{e m}}{2 \pi}\right)
$$

which is the result first obtained by Schwinger 45.].

To remove the UV divergence from the vertex correction Eq. (A10), one has to determine the vertex renormalization constant $Z_{1}$ of Eq. (A6). $Z_{1}$ is determined by requiring that the total vertex

$$
\tilde{\Lambda}^{\mu}=\Lambda^{\mu}+\left(Z_{1}-1\right) \gamma^{\mu},
$$

defines the physical electron charge at $Q^{2}=0$, i.e.

$$
\begin{aligned}
& Z_{1}=1-F\left(Q^{2}=0\right) \\
& =1-\frac{e^{2}}{(4 \pi)^{2}}\left\{\left[\frac{1}{\varepsilon_{U V}}-\gamma_{E}+\ln \left(\frac{4 \pi \mu^{2}}{m^{2}}\right)\right]+2\left[\frac{1}{\varepsilon_{I R}}-\gamma_{E}+\ln \left(\frac{4 \pi \mu^{2}}{m^{2}}\right)\right]+4\right\}+O\left(e^{4}\right) .
\end{aligned}
$$


It is seen that the vertex renormalization constant $Z_{1}$ contains besides the UV divergence also an IR divergence. The renormalized vertex of Eq. (A15), is determined by the vertex correction function $F\left(Q^{2}\right)-F\left(Q^{2}=0\right)$ which is given to first order in $\alpha_{e m}$ (where $\alpha_{e m}=$ $\left.e^{2} / 4 \pi\right)$ by the expression

$$
\begin{gathered}
F\left(Q^{2}\right)-F\left(Q^{2}=0\right)=\frac{\alpha_{e m}}{2 \pi}\left\{\left[\frac{1}{\varepsilon_{I R}}-\gamma_{E}+\ln \left(\frac{4 \pi \mu^{2}}{m^{2}}\right)\right] \cdot\left[\frac{v^{2}+1}{2 v} \ln \left(\frac{v+1}{v-1}\right)-1\right]\right. \\
+\frac{v^{2}+1}{4 v} \ln \left(\frac{v+1}{v-1}\right) \ln \left(\frac{v^{2}-1}{4 v^{2}}\right)+\frac{2 v^{2}+1}{2 v} \ln \left(\frac{v+1}{v-1}\right)-2 \\
\left.+\frac{v^{2}+1}{2 v}\left[S p\left(\frac{v+1}{2 v}\right)-S p\left(\frac{v-1}{2 v}\right)\right]\right\}
\end{gathered}
$$

The expression for the vertex correction function $F\left(Q^{2}\right)-F\left(Q^{2}=0\right)$, which was calculated here using the dimensional regularization method for both the UV and IR divergences, agrees with the ones derived in many textbooks (see e.g. Eq. (47.52) of Ref. [46] where a

full derivation is given). The correspondence with the calculations which use a finite photon mass $(\lambda)$ as IR regulator is found to be

$$
\frac{1}{\epsilon_{I R}}-\gamma_{E}+\ln \left(\frac{4 \pi \mu^{2}}{m^{2}}\right) \longleftrightarrow \ln \frac{\lambda^{2}}{m^{2}} .
$$

In the ultrarelativistic limit $\left(Q^{2}>>m^{2}\right)$, the vertex correction $F\left(Q^{2}\right)-F\left(Q^{2}=0\right)$ can be found from Eq. (A17) to be given by

$$
\begin{aligned}
F\left(Q^{2}\right)-F\left(Q^{2}=0\right) \stackrel{Q^{2}>m^{2}}{\longrightarrow} \frac{\alpha_{e m}}{2 \pi} & \left\{\left[\frac{1}{\varepsilon_{I R}}-\gamma_{E}+\ln \left(\frac{4 \pi \mu^{2}}{m^{2}}\right)\right] \cdot\left[\ln \left(\frac{Q^{2}}{m^{2}}\right)-1\right]\right. \\
& \left.+\left(\frac{3}{2} \ln \left(\frac{Q^{2}}{m^{2}}\right)-2\right)+\left(-\frac{1}{2} \ln ^{2}\left(\frac{Q^{2}}{m^{2}}\right)+\frac{\pi^{2}}{6}\right)\right\} .
\end{aligned}
$$

It is seen from Eq. (A19), that the finite part of the vertex correction at high $Q^{2}$ is dominated by a quadratic logarithmic term.

\section{Lepton self-energy diagram}

The free lepton propagator (for a lepton with four-momentum $k$ )

$$
S^{o}(k)=\frac{\not k+m}{k^{2}-m^{2}+i \epsilon},
$$

is modified through the lepton self-energy $\Sigma(k)$, to the full lepton propagator

$$
S(k)=S^{o}(k)+S^{o}(k) \Sigma(k) S(k) .
$$

To first order $O\left(e^{2}\right)$, the lepton self-energy (corresponding with Fig.19(b)) is given by

$$
-i \Sigma(k)=-e^{2} \mu^{4-D} \int \frac{d^{D} l}{(2 \pi)^{D}} \frac{\gamma^{\alpha}(\not k+\not l+m) \gamma_{\alpha}}{\left[l^{2}\right]\left[(k+l)^{2}-m^{2}\right]} .
$$


By power counting, it is seen that the integral of Eq. (A22) contains a linear UV divergence but is IR finite in the limit $D \rightarrow 4$. The integral of Eq. (A22) can be worked out and yields

$$
\begin{aligned}
\Sigma(k)=-\frac{e^{2}}{(4 \pi)^{2}} & \left\{\left[\frac{1}{\varepsilon_{U V}}-\gamma_{E}+\ln \left(\frac{4 \pi \mu^{2}}{m^{2}}\right)\right](\not k-4 m)\right. \\
& +\not k\left[1+\frac{1}{\tilde{k}^{2}}+\frac{1+\tilde{k}^{2}}{\left(\tilde{k}^{2}\right)^{2}}\left(1-\tilde{k}^{2}\right) \ln \left(1-\tilde{k}^{2}\right)\right] \\
& \left.+2 m\left[-3-\frac{2}{\tilde{k}^{2}}\left(1-\tilde{k}^{2}\right) \ln \left(1-\tilde{k}^{2}\right)\right]\right\},
\end{aligned}
$$

where $\tilde{k}^{2}=k^{2} / m^{2}$.

To remove the UV divergence from the self-energy Eq. (A23), one has to determine the renormalization constants $Z_{2}$ and $Z_{m}$ from Eq. (A6). This counterterm contribution leads to the renormalized self-energy

$$
\tilde{\Sigma}(k)=\Sigma(k)-\left(Z_{2}-1\right) \not k+\left(Z_{2} Z_{m}-1\right) m .
$$

Inserting Eq. (A24) into Eq. (A21) and developing $\Sigma(k)$ as a Taylor series expansion around $\not k=m$ yields for inverse of the total lepton propagator

$$
\begin{aligned}
S^{-1}= & (\not k-m)\left[1-\left.\frac{d \Sigma}{d \not k}\right|_{\not k=m}+\left(Z_{2}-1\right)\right]+\left[\left(1-Z_{m}\right) Z_{2} m-\Sigma(\not k=m)\right] \\
& +O\left((\not k-m)^{2}\right) .
\end{aligned}
$$

Requiring that the total propagator $S$ has a pole at $k=m$ with residue 1 , determines the renormalization constants $Z_{2}$ and $Z_{m}$ as

$$
\begin{array}{r}
Z_{2}=1+\left.\frac{d \Sigma}{d \not k}\right|_{\not k=m}, \\
\left(1-Z_{m}\right) Z_{2} m=\Sigma(\not k=m) .
\end{array}
$$

Using the first order expression of Eq. (A23) for the lepton self-energy, yields

$$
\begin{aligned}
& Z_{2}=1-\frac{e^{2}}{(4 \pi)^{2}}\left\{\left[\frac{1}{\varepsilon_{U V}}-\gamma_{E}+\ln \left(\frac{4 \pi \mu^{2}}{m^{2}}\right)\right]\right. \\
&\left.+2\left[\frac{1}{\varepsilon_{I R}}-\gamma_{E}+\ln \left(\frac{4 \pi \mu^{2}}{m^{2}}\right)\right]+4\right\}+O\left(e^{4}\right), \\
& Z_{2} Z_{m}=1-\frac{e^{2}}{(4 \pi)^{2}}\left\{4\left[\frac{1}{\varepsilon_{U V}}-\gamma_{E}+\ln \left(\frac{4 \pi \mu^{2}}{m^{2}}\right)\right]\right. \\
&\left.+2\left[\frac{1}{\varepsilon_{I R}}-\gamma_{E}+\ln \left(\frac{4 \pi \mu^{2}}{m^{2}}\right)\right]+8\right\}+O\left(e^{4}\right) .
\end{aligned}
$$

Remark that although the unrenormalized lepton self-energy $\Sigma(k)$ of Eq. (A22) is IR finite, the lepton field renormalization constant $Z_{2}$ contains an infrared divergence for the derivative 
of $\Sigma$ that appears in its definition (see Eq. (A27)). Furthermore, a comparison of the first order expressions for the lepton field renormalization constant $Z_{2}$ (Eq. (A28)) with the vertex renormalization constant $Z_{1}$ (Eq. (A16)) shows that they are the same (It is known as a Ward identity and can be shown to hold to all orders as a consequence of the gauge invariance of QED).

Finally, using the expressions of Eqs. (A28), (A29), the renormalized lepton self-energy to first order in $\alpha_{e m}$ is given by

$$
\begin{aligned}
\tilde{\Sigma}(k)=-\frac{\alpha_{e m}}{4 \pi}\left\{k\left[-2\left(\frac{1}{\varepsilon_{I R}}-\gamma_{E}+\ln \left(\frac{4 \pi \mu^{2}}{m^{2}}\right)\right)-3+\frac{1}{\tilde{k}^{2}}+\frac{\left(1-\tilde{k}^{4}\right)}{\tilde{k}^{4}} \ln \left(1-\tilde{k}^{2}\right)\right]\right. \\
\left.-m\left[-2\left(\frac{1}{\varepsilon_{I R}}-\gamma_{E}+\ln \left(\frac{4 \pi \mu^{2}}{m^{2}}\right)\right)-2+\frac{4}{\tilde{k}^{2}}\left(1-\tilde{k}^{2}\right) \ln \left(1-\tilde{k}^{2}\right)\right]\right\} .
\end{aligned}
$$

It is seen from Eq. (A30) that for an on-shell lepton $(\not k=m)$, the renormalized lepton selfenergy $\tilde{\Sigma}$ is exactly zero. Consequently, this correction has only to be applied for internal lepton lines.

\section{Vacuum polarization diagram}

Starting form the free propagator of a photon with four-momentum $q$ (as stated before, we give all expressions in the Feynman gauge)

$$
D_{o}^{\mu \nu}(q)=\frac{-g^{\mu \nu}}{q^{2}}
$$

the full photon propagator can be written as

$$
D^{\mu \nu}(q)=D_{o}^{\mu \nu}(q)+D^{\mu \kappa}(q) \Pi_{\kappa \lambda}(q) D_{o}^{\lambda \nu}(q)
$$

where $\Pi_{\kappa \lambda}(q)$ represents the vacuum polarization correction. To order $O\left(e^{2}\right)$, the vacuum polarization (corresponding with Fig.19(c)) due to $l^{+} l^{-}$loops (with lepton $l=e, \mu, \tau$ ) is given by

$$
-i \Pi^{\mu \nu}(q)=-e^{2} \mu^{4-D} \int \frac{d^{D} l}{(2 \pi)^{D}} \frac{\operatorname{Tr}\left\{\gamma^{\mu}(\not l+\not q+m) \gamma^{\nu}(\not l+m)\right\}}{\left[(l+q)^{2}-m^{2}\right]\left[l^{2}-m^{2}\right]}+O\left(e^{4}\right) .
$$

The gauge invariance of QED leads to the relation $q^{\kappa} q^{\lambda} \Pi_{\kappa \lambda}(q)=0$ (Ward-Takahashi identity). Consequently, the vacuum polarization correction can be written as

$$
\Pi_{\kappa \lambda}(q)=\left(-g_{\kappa \lambda} q^{2}+q_{\kappa} q_{\lambda}\right) \Pi\left(q^{2}\right),
$$

where the function $\Pi\left(q^{2}\right)$ is IR convergent and contains only a logarithmic UV divergence as can be seen from Eq. (A33).

Using Eq. (A34), the self-consistent relation for the full photon propagator (Eq. (A32)) yields

$$
D^{\mu \nu}(q)=\frac{-g^{\mu \nu}}{q^{2}\left(1-\Pi\left(q^{2}\right)\right)}+\operatorname{term} \operatorname{in} q^{\mu} q^{\nu}
$$


where we don't have to specify the term in $q^{\mu} q^{\nu}$, as the photon propagator will be contracted with conserved currents on both sides so that this term in $q^{\mu} q^{\nu}$ will not contribute to physical observables. Evaluating the one-loop integral of Eq. (A33), one obtains

$$
\Pi\left(Q^{2}\right)=-\frac{e^{2}}{(4 \pi)^{2}} \frac{4}{3}\left[\frac{1}{\varepsilon_{U V}}-\gamma_{E}+\ln \left(\frac{4 \pi \mu^{2}}{m^{2}}\right)-\left(v^{2}-\frac{8}{3}\right)+v \frac{\left(v^{2}-3\right)}{2} \ln \left(\frac{v+1}{v-1}\right)\right],
$$

where $v$ is given by Eq. (A12).

The UV divergent term in Eq. (A36) is removed by adding the counterterm in $Z_{3}$ of Eq. (A6). This leads to the renormalized photon propagator

$$
\tilde{D}^{\mu \nu}(q)=\frac{-g^{\mu \nu}}{q^{2}\left(1-\tilde{\Pi}\left(q^{2}\right)\right)}+\operatorname{term} \text { in } q^{\mu} q^{\nu}
$$

where the renormalized photon polarization $\tilde{\Pi}$ is given by

$$
\tilde{\Pi}\left(Q^{2}\right)=\Pi\left(Q^{2}\right)-\left(Z_{3}-1\right) .
$$

Requiring that the renormalized photon propagator (Eq. (A35) has a pole at $q^{2}=0$ with residue 1 , determines the renormalization constant $Z_{3}$ :

$$
Z_{3}=1+\Pi\left(q^{2}=0\right) .
$$

Consequently, the renormalized finite photon polarization is found from Eqs. A36) and (A38) to be given by

$$
\tilde{\Pi}\left(Q^{2}\right)=\frac{\alpha_{e m}}{\pi} \frac{1}{3}\left[\left(v^{2}-\frac{8}{3}\right)+v \frac{\left(3-v^{2}\right)}{2} \ln \left(\frac{v+1}{v-1}\right)\right],
$$

which agrees with the result derived in Ref. [46].

\section{Soft photon emission contributions}

The calculation of the one-loop vertex correction of Eq. (A9) was seen to be both UV and IR divergent. The ultraviolet divergence was removed by renormalizing the fields and parameters of the theory. The remaining infrared divergences are cancelled at the cross section level by the soft bremsstrahlung contributions [26,27].

In this bremsstralung process (see Figs.19 (d) and (e)), an electron is accompanied by the emission of a soft photon of maximal energy $\Delta E_{s}$ (which is related to the detector resolution and is therefore much smaller than the electron energy which radiates this soft photon). To first order in $\alpha_{e m}$ (relative to the Born cross section) the bremsstrahlung cross section amounts to calculate a phase space integral of the form :

$$
\begin{aligned}
& d \sigma \sim \frac{d^{3} \vec{k}_{e}^{\prime}}{(2 \pi)^{3} 2 E_{e}^{\prime}} \frac{d^{3} \vec{p}_{N}^{\prime}}{(2 \pi)^{3} 2 E_{N}^{\prime}} \frac{d^{3} \vec{l}}{(2 \pi)^{3} 2 \mathrm{l}}(2 \pi)^{4} \delta^{4}\left(k+p-k^{\prime}-p^{\prime}-l\right) \\
& \times\left|M_{B O R N}\right|^{2}\left(-e^{2}\right)\left[\frac{k_{\mu}^{\prime}}{k^{\prime} . l}-\frac{k_{\mu}}{k . l}\right] \cdot\left[\frac{k^{\prime \mu}}{k^{\prime} . l}-\frac{k^{\mu}}{k . l}\right],
\end{aligned}
$$


where $l \equiv|\vec{l}|$ denotes the soft photon energy, and where $M_{B O R N}$ denotes the Born amplitude for elastic lepton-nucleon scattering. In Eq. (A41), terms in the soft photon momentum were neglected compared with the electron momenta $k$ and $k^{\prime}$, except in the denominators of the lepton propagators where they matter.

If one performs an experiment where the outgoing electron is detected, and where the recoiling proton remains undetected (i.e. if one measures a single arm electron spectrum), one eliminates in Eq. (A41) the integral over $\vec{p}_{N}^{\prime}$ with the momentum conserving $\delta$-function, which gives :

$$
\begin{aligned}
& d \sigma \sim \frac{d^{3} \vec{k}_{e}^{\prime}}{(2 \pi)^{3} 2 E_{e}^{\prime}} \frac{d^{3} \vec{l}}{(2 \pi)^{3} 21} \frac{1}{2 E_{N}^{\prime}}(2 \pi) \delta\left(E_{e}+E_{N}-E_{e}^{\prime}-\sqrt{\left(\vec{q}+\vec{p}_{N}-\vec{l}\right)^{2}+M_{N}^{2}}-1\right) \\
& \times\left|M_{B O R N}\right|^{2}\left(-e^{2}\right)\left[\frac{k_{\mu}^{\prime}}{k^{\prime} . l}-\frac{k_{\mu}}{k . l}\right] \cdot\left[\frac{k^{\prime \mu}}{k^{\prime} . l}-\frac{k^{\mu}}{k . l}\right] .
\end{aligned}
$$

Due the energy conserving $\delta$-function in Eq. (A42), the integration volume for the soft photon has a complicated ellipsoidal shape in the lab system. In order for the soft-photon phase space integration volume to be spherical, one has to perform the calculation in the c.m. system $\mathcal{S}$ of the (recoiling nucleon + soft-photon), as discussed in [21]. The system $\mathcal{S}$ is thus defined by : $\vec{p}_{N}^{\prime}+\vec{l}=\vec{q}+\vec{p}_{N}=0$. In the system $\mathcal{S}$, the energy conserving delta function is independent of the soft-photon angles, and the maximal soft photon energy is isotropic. The integral over the soft-photon momentum (up to some maximum value $\Delta E_{s}$ ) can then be performed independently from the integration over the soft photon emission angles. If $\Delta E_{s}$ is sufficiently small, one can furthermore neglect the soft photon energy with respect to the other energies in the $\delta$-function, and perform the integral over the electron momentum $\left|\vec{k}_{e}^{\prime}\right|$ in Eq. ( $\mathrm{A} 42$ ). The integration over the outgoing electron momentum eliminates the $\delta$ function, which implies the elastic scattering constraint. This yields then for the differential cross section with respect to the outgoing electron angles, the following correction due to soft bremsstrahlung :

$$
\left(\frac{d \sigma}{d \Omega_{e}^{\prime}}\right)_{R E A L S O F T \gamma}=\left(\frac{d \sigma}{d \Omega_{e}^{\prime}}\right)_{B O R N}\left(-e^{2}\right) \int \frac{d^{3} \vec{l}}{(2 \pi)^{3} 2 l}\left[\frac{k_{\mu}^{\prime}}{k^{\prime} . l}-\frac{k_{\mu}}{k . l}\right] \cdot\left[\frac{k^{\prime \mu}}{k^{\prime} . l}-\frac{k^{\mu}}{k . l}\right],
$$

where the soft-photon phase space integral is performed in the system $\mathcal{S}$, in which the integration volume is spherical. We will denote in the following the external kinematics in the system $\mathcal{S}$ by tilded quantities $\left(\tilde{E}_{e}, \tilde{E}_{e}^{\prime}, \tilde{E}_{N}, \tilde{E}_{N}^{\prime}\right)$ to distinguish them from the lab quantities, which we denote by untilded quantities $\left(E_{e}, E_{e}^{\prime}, E_{N} \equiv M_{N}, E_{N}^{\prime}\right)$. To make the transformation between the system $\mathcal{S}$ and the lab system, we first introduce the missing four-momentum $p_{m} \equiv p_{N}^{\prime}+l$. The system $\mathcal{S}$ is defined by $\vec{p}_{m}=\overrightarrow{0}$, and the soft photon limit implies $p_{m}^{0} \approx M_{N}$. We can then easily express in the system $\mathcal{S}$, the energies for the external particles in the elastic scattering process, in terms of lab quantities :

$$
\begin{aligned}
& \tilde{E}_{e} \approx \frac{k \cdot p_{m}}{M_{N}}=\frac{1}{M_{N}} k \cdot(p+q)=\frac{1}{M_{N}}\left(M_{N} E_{e}-Q^{2} / 2\right)=E_{e}^{\prime}, \\
& \tilde{E}_{e}^{\prime} \approx \frac{k^{\prime} \cdot p_{m}}{M_{N}}=\frac{1}{M_{N}} k^{\prime} \cdot(p+q)=\frac{1}{M_{N}}\left(M_{N} E_{e}^{\prime}+Q^{2} / 2\right)=E_{e}, \\
& \tilde{E}_{N} \approx \frac{p \cdot p_{m}}{M_{N}}=\frac{1}{M_{N}} p \cdot(p+q)=M_{N}+E_{e}-E_{e}^{\prime}=E_{N}^{\prime},
\end{aligned}
$$


where the elastic scattering condition $\left(Q^{2}=2 M_{N}\left(E_{e}-E_{e}^{\prime}\right)\right)$ has been used in the last step in Eqs. (A44, A45). The angle $\tilde{\theta}_{e}$ in the frame $\mathcal{S}$ is obtained from $k \cdot k^{\prime}=\tilde{E}_{e} \tilde{E}_{e}^{\prime}\left(1-\cos \tilde{\theta}_{e}\right)=$ $E_{e} E_{e}^{\prime}\left(1-\cos \theta_{e}\right)$, which shows (using Eqs. (A44,A45)) that in the soft-photon limit, this angle is the same as in the lab system, i.e. $\cos \theta_{e}=\cos \theta_{e}$.

The integral of Eq. A43) extends up to a maximal soft-photon energy $\Delta E_{s}$ in the system $\mathcal{S}$, which is expressed in terms of the $l a b$ quantities $E_{e}$ and $E_{e}^{\prime}$, by using :

$$
\left(p^{\prime}+l\right)^{2}-M_{N}^{2}=\left(p+k-k^{\prime}\right)^{2}-M_{N}^{2}=2 p \cdot\left(k-k^{\prime}\right)+\left(k-k^{\prime}\right)^{2},
$$

which leads (for soft-photon energies, i.e. keeping only terms of first order in $\Delta E_{s}$ ) to

$$
\begin{aligned}
2 M_{N} \Delta E_{s} & \approx 2 M_{N}\left(E_{e}-E_{e}^{\prime}\right)-4 E_{e} E_{e}^{\prime} \sin ^{2} \theta_{e} / 2, \\
& =2 M_{N}\left(E_{e}-E_{e}^{\prime}\right)-2 M_{N}\left(E_{e}-E_{e}^{\prime e l}\right) E_{e}^{\prime} / E_{e}^{\prime e l} .
\end{aligned}
$$

All quantities on the rhs of Eq. (A48) are in the lab, and the elastic scattering condition has been used in the last line $\left(E_{e}^{\prime e l}\right.$ denotes the elastic scattered electron lab energy, to distinguish it from $E_{e}^{\prime}$ ). From Eq. (A48), one determines then $\Delta E_{s}$ in terms of lab quantities from the scattered electron spectrum through

$$
\Delta E_{s}=\eta\left(E_{e}^{\prime e l}-E_{e}^{\prime}\right)
$$

where the recoil factor $\eta$ is given by $\eta=E_{e} / E_{e}^{\prime e l}$.

Deviations from the soft-photon emission formula Eq. (A43) will show up when $\Delta E_{s}$ is not very small compared with the lepton momenta in the process. The emission of such a semi-hard photon is what is usually referred to as the radiative tail. Although the distinction is somewhat arbitrary, one can always split the integral for photon emission into two parts, one by integrating up to a small value $\Delta E_{s}$, where the soft-photon approximation in writing down Eq. (A43) holds, and a second integral, starting from this small (but non-zero) value of $\Delta E_{s}$ up to the energy where one performs the cut in the spectrum. This second integral is finite and can be performed numerically. Such a numerical calculation of the radiative tail without approximations is presented in section IV. In the present section, we give an analytical result for the soft-photon (i.e. small $\Delta E_{s}$ ) integral of Eq. (A43), without any further approximations (Remark that in [22] only an approximate evaluation of Eq. (A43) has been given).

As is immediately seen by power counting, the integral in Eq. (A43) has a logarithmic IR divergence, corresponding with the emission of photons with zero energy. To demonstrate the cancellation with the IR divergence of the vertex diagram as stated above, one has to regularize the integral of Eq. (A43). In this work this is performed by also using dimensional regularization. The soft photon integral is then evaluated in $D-1$ dimensions $(D \rightarrow 4$ corresponds to the physical limit). One now sees that it is extremely advantageous to have a spherical integration volume, in order to evaluate the integral for dimensions $D \neq 4$. Before continuing the integral of Eq. (A43) into $D-1$ dimensions, the integration limits for $l$ have to be made dimensionless, which leads in the dimensional regularization scheme to the introduction of the same scale $\mu$ in Eq. (A50) as was introduced when changing the dimension of the virtual photon loop integral of Eq. (A9). This leads then in $D-1$ dimensions, to the bremsstrahlung integral : 


$$
I=-e^{2} \int^{1<\Delta E_{s} / \mu} \frac{d^{D-1} l}{(2 \pi)^{D-1} 21}\left[\frac{k_{\alpha}^{\prime}}{k^{\prime} . l}-\frac{k_{\alpha}}{k . l}\right] \cdot\left[\frac{k^{\prime \alpha}}{k^{\prime} . l}-\frac{k^{\alpha}}{k . l}\right] .
$$

The integral in Eq. (A50) is worked out by introducing polar coordinates in $D-1$ dimensions. To define the polar angle in the interference term of Eq. (A50), a Feynman parametrization is performed. This leads for $I$ to the expression :

$$
\begin{aligned}
I= & e^{2} \int_{0}^{1<\Delta E_{s} / \mu} \frac{d l}{(2 \pi)^{D-1}} \frac{l^{D-2}}{2 l^{3}} \\
& \times \int_{D-2} d \Omega_{l}\left\{\frac{k \cdot k^{\prime}}{\tilde{E}_{e} \tilde{E}_{e}^{\prime}} \int_{-1}^{+1} d y \frac{1}{\left(1-\tilde{\vec{\beta}}_{y} \cdot \hat{l}\right)^{2}}-\frac{\left(1-\tilde{\beta}_{e}^{2}\right)}{\left(1-\tilde{\vec{\beta}}_{e} \cdot \hat{l}\right)^{2}}-\frac{\left(1-\tilde{\beta}_{e}^{2}\right)}{\left(1-\tilde{\vec{\beta}}_{e}^{\prime} \cdot \hat{l}\right)^{2}}\right\},
\end{aligned}
$$

where $\hat{l}$ is the unit-vector along the soft photon direction, $\tilde{\beta}_{e} \equiv\left|\tilde{\vec{\beta}}_{e}\right|, \tilde{\beta}_{e}^{\prime} \equiv\left|\tilde{\vec{\beta}}_{e}^{\prime}\right|$ are the incoming and outgoing electron velocities (in the system $\mathcal{S}$ ) respectively and where $\tilde{\beta}_{y} \equiv\left|\tilde{\vec{\beta}}_{y}\right|$ with

$$
\begin{aligned}
\tilde{\vec{\beta}_{e}} & \equiv \frac{\tilde{\vec{k}_{e}}}{\tilde{E}_{e}}, \quad \quad \tilde{\vec{\beta}}_{e}^{\prime} \equiv \frac{\tilde{\vec{k}}_{e}^{\prime}}{\tilde{E}_{e}^{\prime}}, \\
\tilde{\vec{\beta}}_{y} & \equiv \tilde{\vec{\beta}}_{e} \frac{1}{2}(1+y)+\tilde{\vec{\beta}}_{e}^{\prime} \frac{1}{2}(1-y) .
\end{aligned}
$$

The integrals over 1 and the azimuthal angular integral (over $D-2$ dimensions) can be performed immediately which yields :

$$
\begin{aligned}
I=e^{2}[ & \left.\frac{(2 \pi)^{2 \epsilon_{I R}}}{(2 \pi)^{3}}\left(\frac{\Delta E_{s}}{\mu}\right)^{-2 \epsilon_{I R}} \frac{1}{-4 \epsilon_{I R}}\right] \cdot\left[\frac{2 \pi}{\pi^{\epsilon_{I R}}} \frac{1}{\Gamma\left(1-\epsilon_{I R}\right)}\right] \\
\times & \left\{\frac{k \cdot k^{\prime}}{\tilde{E}_{e} \tilde{E}_{e}^{\prime}} \int_{-1}^{+1} d y \int_{-1}^{+1} d x \frac{\left(1-x^{2}\right)^{-\epsilon_{I R}}}{\left(1-\tilde{\beta}_{y} x\right)^{2}}\right. \\
& \left.-\left(1-\tilde{\beta}_{e}^{2}\right) \int_{-1}^{+1} d x \frac{\left(1-x^{2}\right)^{-\epsilon_{I R}}}{\left(1-\tilde{\beta}_{e} x\right)^{2}}-\left(1-\tilde{\beta}_{e}^{\prime 2}\right) \int_{-1}^{+1} d x \frac{\left(1-x^{2}\right)^{-\epsilon_{I R}}}{\left(1-\tilde{\beta}_{e}^{\prime} x\right)^{2}}\right\},
\end{aligned}
$$

The IR divergent term and the finite term are obtained by developing the polar angular integral in Eq. (A53) as

$$
\int_{-1}^{+1} d x \frac{\left(1-x^{2}\right)^{-\epsilon_{I R}}}{(1-\beta x)^{2}}=\int_{-1}^{+1} d x \frac{1}{(1-\beta x)^{2}}-\epsilon_{I R} \int_{-1}^{+1} d x \frac{\ln \left(1-x^{2}\right)}{(1-\beta x)^{2}}+O\left(\epsilon_{I R}^{2}\right) .
$$

Performing the integrations in Eq. (A54) (the second integral in Eq. (A54) is simplified by making the substitution $x \rightarrow u=\beta /(1-\beta x))$ yields

$$
\int_{-1}^{+1} d x \frac{\left(1-x^{2}\right)^{-\epsilon_{I R}}}{(1-\beta x)^{2}}=\frac{2}{1-\beta^{2}}-\epsilon_{I R} \frac{2}{1-\beta^{2}}\left[\ln 4+\frac{1}{\beta} \ln \frac{1-\beta}{1+\beta}\right]+O\left(\epsilon_{I R}^{2}\right) .
$$


Consequently, the IR divergent term and the finite term of the integral $I$ are obtained by using Eq. (A55) in Eq. (A53) and by developing all other factors also to order $\epsilon_{I R}$ :

$$
\begin{aligned}
I=-\frac{e^{2}}{4 \pi^{2}}\left\{\left[-\frac{1}{\epsilon_{I R}}+\gamma_{E}-\ln \frac{4 \pi \mu^{2}}{m^{2}}+\ln \frac{4\left(\Delta E_{s}\right)^{2}}{m^{2}}\right]\left[1-\frac{1}{2}\left(1-\tilde{\beta}_{e} \tilde{\beta}_{e}^{\prime} \cos \tilde{\theta}_{e}\right) I_{y}^{(1)}\right]\right. \\
\left.+\left[\frac{1}{2 \tilde{\beta}_{e}} \ln \frac{1-\tilde{\beta}_{e}}{1+\tilde{\beta}_{e}}+\frac{1}{2 \tilde{\beta}_{e}^{\prime}} \ln \frac{1-\tilde{\beta}_{e}^{\prime}}{1+\tilde{\beta}_{e}^{\prime}}-\frac{1}{2}\left(1-\tilde{\beta}_{e} \tilde{\beta}_{e}^{\prime} \cos \tilde{\theta}_{e}\right) I_{y}^{(2)}\right]\right\},
\end{aligned}
$$

where the remaining Feynman parameter integrals $I_{y}^{(1)}$ and $I_{y}^{(2)}$ are given by

$$
\begin{aligned}
I_{y}^{(1)} & \equiv \int_{-1}^{+1} d y \frac{1}{1-\tilde{\beta}_{y}^{2}} \\
I_{y}^{(2)} & \equiv \int_{-1}^{+1} d y \frac{1}{\tilde{\beta}_{y}\left(1-\tilde{\beta}_{y}^{2}\right)} \ln \frac{1-\tilde{\beta}_{y}}{1+\tilde{\beta}_{y}}
\end{aligned}
$$

and where $\tilde{\beta}_{y}$ is given by Eq. (A52). The integral $I_{y}^{(1)}$ in Eq. (A57) can be performed easily and yields

$$
I_{y}^{(1)}=\frac{2 \tilde{E}_{e} \tilde{E}_{e}^{\prime}}{m^{2}} \frac{v^{2}-1}{2 v} \ln \left(\frac{v+1}{v-1}\right),
$$

with $v$ as defined in Eq. (A12). To obtain an analytical formula for the integral $I_{y}^{(2)}$ is much harder but was performed in Ref. [47, which we checked ${ }^{2}$ and which yields the result:

$$
\begin{aligned}
I_{y}^{(2)}=\frac{1}{\left|\tilde{\vec{\beta}}_{e}-\tilde{\vec{\beta}}_{e}^{\prime}\right| \tanh \alpha}\{ & {\left[-2 \ln (2)+\frac{1}{2} \ln \left(\sinh ^{2} \alpha-\sinh ^{2} \phi_{1}\right)\right] \ln \frac{\sinh \alpha+\sinh \phi_{1}}{\sinh \alpha-\sinh \phi_{1}} } \\
& -\ln \left(\sinh \alpha+\sinh \phi_{1}\right) \ln \frac{\sinh \alpha-\sinh \phi_{1}}{4 \sinh ^{2} \alpha} \\
& +2 \ln \left[e^{-\alpha} \frac{e^{\alpha}+e^{\phi_{1}}}{e^{-\alpha}+e^{\phi_{1}}}\right] \ln \frac{\cosh \alpha+\cosh \phi_{1}}{\cosh \alpha-\cosh \phi_{1}} \\
& -2 \Phi\left[\frac{\sinh \alpha+\sinh \phi_{1}}{2 \sinh \alpha}\right]+\Phi\left[\left(\frac{e^{\alpha}-e^{\phi_{1}}}{e^{\alpha}+e^{\phi_{1}}}\right)^{2}\right] \\
& \left.-\Phi\left[\left(\frac{e^{\phi_{1}}-e^{-\alpha}}{e^{\phi_{1}}+e^{-\alpha}}\right)^{2}\right]-\left[\phi_{1} \longrightarrow \phi_{2}\right]\right\}
\end{aligned}
$$

where $\alpha, \phi_{1}$ and $\phi_{2}$ are given by :

$$
\cosh \alpha=\frac{\left|\tilde{\vec{\beta}}_{e}-\tilde{\vec{\beta}}_{e}^{\prime}\right|}{\tilde{\beta}_{e} \tilde{\beta}_{e}^{\prime} \sin \tilde{\theta}_{e}} \quad(\alpha>0),
$$

\footnotetext{
${ }^{2}$ Note that the relevant formula quoted in Ref. [47] contains some typing errors.
} 


$$
\begin{aligned}
\cosh \phi_{1} & =\tilde{\beta}_{e} \cosh \alpha, & \sinh \phi_{1} & =\frac{-\tilde{\beta}_{e} \tilde{\beta}_{e}^{\prime} \cos \tilde{\theta}_{e}+\tilde{\beta}_{e}^{2}}{\tilde{\beta}_{e} \tilde{\beta}_{e}^{\prime} \sin \tilde{\theta}_{e}}, \\
\cosh \phi_{2} & =\tilde{\beta}_{e}^{\prime} \cosh \alpha, & \sinh \phi_{2} & =\frac{\tilde{\beta}_{e} \tilde{\beta}_{e}^{\prime} \cos \tilde{\theta}_{e}-\tilde{\beta}_{e}^{\prime 2}}{\tilde{\beta}_{e} \tilde{\beta}_{e}^{\prime} \sin \tilde{\theta}_{e}}
\end{aligned}
$$

The function $\Phi$ in Eq. (A59) is given by

$$
\Phi(x) \equiv-\int_{0}^{x} d t \frac{\ln |1-t|}{t} .
$$

which agrees with the Spence function (Eq. (A13)) when $x<1$. Compared with previous calculations in the literature, it was shown in Ref. [47] that this integral $I_{y}^{(2)}$ was approximated in Ref. [48] and that the calculation of this integral in Ref. 49 contains a factor two error. We also checked the analytical formula of Eq. (A59) by performing the integral of Eq. (A57) numerically.

In the ultrarelativistic limit $\left(\tilde{\beta}_{e}, \tilde{\beta}_{e}^{\prime} \rightarrow 1\right)$, the integral $I_{y}^{(2)}$ of Eq. (A59) reduces to

$$
\begin{aligned}
I_{y}^{(2)} \stackrel{\tilde{\beta}_{e} \approx 1, \tilde{\beta}_{e}^{\prime} \approx 1}{\longrightarrow} \frac{1}{2 \sin ^{2} \frac{\tilde{\theta}_{e}}{2}} & \left\{-\frac{1}{2} \ln ^{2}\left(1-\tilde{\beta}_{e}^{2}\right)-\frac{1}{2} \ln ^{2}\left(1-\tilde{\beta}_{e}^{\prime 2}\right)+\ln 4 \ln \left(1-\tilde{\beta}_{e}^{2}\right)+\ln 4 \ln \left(1-\tilde{\beta}_{e}^{\prime 2}\right)\right. \\
+ & 4\left(\ln ^{2}\left(\sin \frac{\tilde{\theta}_{e}}{2}\right)-\ln ^{2} 2\right)-2 \ln \left(\cos ^{2} \frac{\tilde{\theta}_{e}}{2}\right) \ln \left(\sin ^{2} \frac{\tilde{\theta}_{e}}{2}\right) \\
& \left.-\frac{\pi^{2}}{3}-2 \operatorname{Sp}\left(\sin ^{2} \frac{\tilde{\theta}_{e}}{2}\right)\right\} .
\end{aligned}
$$

Putting all pieces together, the result for the bremsstrahlung cross section accompanying elastic electron scattering is obtained as

$$
\begin{aligned}
& \left(\frac{d \sigma}{d \Omega_{e}^{\prime}}\right)_{\text {REALSOFT } \gamma}\left\{\frac{\alpha_{e m}}{\pi}\left[-\frac{1}{\varepsilon_{I R}}+\gamma_{E}-\ln \left(\frac{4 \pi \mu^{2}}{m^{2}}\right)\right]\left[\frac{v^{2}+1}{2 v} \ln \left(\frac{v+1}{v-1}\right)-1\right]+\delta_{R}\right\}, \\
& =\left(\frac{d \sigma}{d \Omega_{e}^{\prime}}\right)_{B O R N}
\end{aligned}
$$

where the finite part $\delta_{R}$ of the real radiative corrections is given by

$$
\begin{aligned}
\delta_{R}=\frac{\alpha_{e m}}{\pi} & \left\{\ln \left(\frac{4\left(\Delta E_{s}\right)^{2}}{m^{2}}\right)\left[\frac{v^{2}+1}{2 v} \ln \left(\frac{v+1}{v-1}\right)-1\right]\right. \\
& \left.-\frac{1}{2 \tilde{\beta}_{e}} \ln \left(\frac{1-\tilde{\beta}_{e}}{1+\tilde{\beta}_{e}}\right)-\frac{1}{2 \tilde{\beta}_{e}^{\prime}} \ln \left(\frac{1-\tilde{\beta}_{e}^{\prime}}{1+\tilde{\beta}_{e}^{\prime}}\right)+\frac{1}{2}\left(1-\tilde{\beta}_{e} \tilde{\beta}_{e}^{\prime} \cos \tilde{\theta}_{e}\right) I_{y}^{(2)}\right\},(A) \\
\stackrel{Q^{2}>m^{2}}{\longrightarrow} \frac{\alpha_{e m}}{\pi} & \left\{\ln \left(\frac{4\left(\Delta E_{s}\right)^{2}}{m^{2}}\right)\left[\ln \left(\frac{Q^{2}}{m^{2}}\right)-1\right]-\frac{1}{2} \ln \left(\frac{1-\tilde{\beta}_{e}^{2}}{4}\right)-\frac{1}{2} \ln \left(\frac{1-\tilde{\beta}_{e}^{\prime 2}}{4}\right)\right. \\
& -\frac{1}{4} \ln ^{2}\left(1-\tilde{\beta}_{e}^{2}\right)-\frac{1}{4} \ln ^{2}\left(1-\tilde{\beta}_{e}^{\prime 2}\right)+\ln 2 \ln \left(1-\tilde{\beta}_{e}^{2}\right)+\ln 2 \ln \left(1-\tilde{\beta}_{e}^{\prime 2}\right)
\end{aligned}
$$




$$
\begin{aligned}
+ & \left.2\left(\ln ^{2}\left(\sin \frac{\tilde{\theta}_{e}}{2}\right)-\ln ^{2} 2\right)-\frac{\pi^{2}}{3}+S p\left(\cos ^{2} \frac{\tilde{\theta}_{e}}{2}\right)\right\}, \\
=\frac{\alpha_{e m}}{\pi} & \left\{\ln \left(\frac{\left(\Delta E_{s}\right)^{2}}{\tilde{E}_{e} \tilde{E}_{e}^{\prime}}\right)\left[\ln \left(\frac{Q^{2}}{m^{2}}\right)-1\right]\right. \\
& \left.-\frac{1}{2} \ln ^{2}\left(\frac{\tilde{E}_{e}}{\tilde{E}_{e}^{\prime}}\right)+\frac{1}{2} \ln ^{2}\left(\frac{Q^{2}}{m^{2}}\right)-\frac{\pi^{2}}{3}+S p\left(\cos ^{2} \frac{\tilde{\theta}_{e}}{2}\right)\right\},
\end{aligned}
$$

where the expression of Eq. (A65) in the $Q^{2}>>m^{2}$ limit has been rewritten in Eq. (A66) to allow comparison with other expressions found in the literature.

Finally to evaluate $\delta_{R}$, we have to express the quantities in the system $\mathcal{S}$ in terms of $l a b$ quantities. The relations given in Eqs. (A44, A45)) yield for elastic scattering : $\tilde{E}_{e}=E_{e}^{\prime}$, $\tilde{E}_{e}^{\prime}=E_{e}$, and $\cos \tilde{\theta}_{e}=\cos \theta_{e}$. From the formula for $\delta_{R}$ (e.g. Eq. (A66), one then sees that one formally obtains exactly the same expression in terms of the lab quantities $E_{e}, E_{e}^{\prime}, \theta_{e}$. The quantity $\Delta E_{s}$ is calculated from the cut in the electron spectrum, using the expression of Eq. (A49).

A comparison of expressions Eqs. (A63), (A64) with the literature, shows that the same result is obtained as in Ref. [47. A comparison with the expression used by Mo and Tsai 222 will be given in the next section when we add the vertex correction and soft photon emission contribution, because only their sum is IR finite (and thus independent of the IR regularization procedure used).

\section{Elastic lepton-nucleon scattering}

In this section, we bring together the first order radiative corrections at the lepton side (lepton vertex and soft bremsstrahlung from the lepton) and the photon polarization correction to correct the elastic lepton-nucleon scattering cross section. As was shown in the previous sections, these corrections can be calculated model-independently. In the next section, we discuss the additional radiative corrections to the lepton-proton cross section, which originate from the proton side (proton vertex correction, soft bremsstrahlung from proton and two-photon exchange corrections). To calculate these corrections at the proton side, a model for the off-shell (or half off-shell) $\gamma N N$ vertex is needed however, and which is therefore to some extent model-dependent. For this latter part, we will refer to the recent work of Ref. [24].

The elastic lepton scattering cross section, corrected to first order in $\alpha_{e m}$ for the lepton vertex contribution and for the photon polarization contribution, is given by

$$
\begin{aligned}
& \left(\frac{d \sigma}{d \Omega_{e}^{\prime}}\right)_{V I R T U A L \gamma} \approx\left(\frac{d \sigma}{d \Omega_{e}^{\prime}}\right)_{B O R N} \frac{1}{\left(1-\tilde{\Pi}\left(Q^{2}\right)\right)^{2}}\left(1+2\left\{F\left(Q^{2}\right)-F\left(Q^{2}=0\right)\right\}\right) \\
= & \left(\frac{d \sigma}{d \Omega_{e}^{\prime}}\right)_{B O R N} \frac{1}{\left(1-\tilde{\Pi}\left(Q^{2}\right)\right)^{2}} \\
& \times\left(1+\frac{\alpha_{e m}}{\pi}\left[\frac{1}{\varepsilon_{I R}}-\gamma_{E}+\ln \left(\frac{4 \pi \mu^{2}}{m^{2}}\right)\right] \cdot\left[\frac{v^{2}+1}{2 v} \ln \left(\frac{v+1}{v-1}\right)-1\right]+\delta_{\text {vertex }}\right),
\end{aligned}
$$


where the finite part $\delta_{\text {vertex }}$ of the lepton vertex correction is found from Eq. (A17) to be given by

$$
\begin{gathered}
\delta_{\text {vertex }}=\frac{\alpha_{e m}}{\pi}\left\{\frac{v^{2}+1}{4 v} \ln \left(\frac{v+1}{v-1}\right) \ln \left(\frac{v^{2}-1}{4 v^{2}}\right)+\frac{2 v^{2}+1}{2 v} \ln \left(\frac{v+1}{v-1}\right)-2\right. \\
\left.+\frac{v^{2}+1}{2 v}\left[S p\left(\frac{v+1}{2 v}\right)-S p\left(\frac{v-1}{2 v}\right)\right]\right\}, \\
\stackrel{Q^{2}>>m^{2}}{\longrightarrow} \frac{\alpha_{e m}}{\pi}\left\{\frac{3}{2} \ln \left(\frac{Q^{2}}{m^{2}}\right)-2-\frac{1}{2} \ln ^{2}\left(\frac{Q^{2}}{m^{2}}\right)+\frac{\pi^{2}}{6}\right\} .
\end{gathered}
$$

In writing down Eq. (A67) to first order in $\alpha_{e m}$, the contribution of the anomalous magnetic moment term $G\left(Q^{2}\right)$ in the vertex correction Eq. (A8) has been dropped. This contribution vanishes in the ultrarelativistic limit $\left(Q^{2}>>m^{2}\right)$ as can be seen from Eq. (A11). The first term in the last line of Eq. (A68) corresponds with the vertex correction term quoted by Mo and Tsai (Eq. ( II.5) of Ref. [22]).

The finite part of the photon polarization correction, $\delta_{v a c} \equiv 2 \tilde{\Pi}\left(Q^{2}\right)$, follows from Eq. ( $\mathrm{A} 40)$ as

$$
\begin{aligned}
\delta_{v a c} & =\frac{\alpha_{e m}}{\pi} \frac{2}{3}\left\{\left(v^{2}-\frac{8}{3}\right)+v \frac{\left(3-v^{2}\right)}{2} \ln \left(\frac{v+1}{v-1}\right)\right\}, \\
\stackrel{Q^{2} \gg>m^{2}}{\longrightarrow} & \frac{\alpha_{e m}}{\pi} \frac{2}{3}\left\{-\frac{5}{3}+\ln \left(\frac{Q^{2}}{m^{2}}\right)\right\},
\end{aligned}
$$

which agrees with the expression quoted by Mo and Tsai (Eq. ( II.4) of Ref. 22]). To evaluate the vacuum polarization due to $\mu^{+} \mu^{-}$and $\tau^{+} \tau^{-}$pairs at intermediate $Q^{2}$, one has to use Eq. (A69) instead of the limit of Eq. (A70). P

When adding the real (Eq. (A63)) and virtual (Eq. (A67)) radiative corrections at the lepton side, one verifies that the IR divergent parts exactly cancel. The remaining finite contribution is given to first order in $\alpha_{e m}$ by

$$
\left(\frac{d \sigma}{d \Omega_{e}^{\prime}}\right)_{V I R T U A L \gamma}+\left(\frac{d \sigma}{d \Omega_{e}^{\prime}}\right)_{R E A L S O F T \gamma}=\left(\frac{d \sigma}{d \Omega_{e}^{\prime}}\right)_{B O R N}\left(1+\delta_{v a c}+\delta_{\text {vertex }}+\delta_{R}\right)
$$

where $\delta_{\text {vac }}, \delta_{\text {vertex }}$ and $\delta_{R}$ are given by Eqs. (A69), (A68), and (A64)-(A66) respectively. Bringing the three contributions together, leads to the expression (in the $Q^{2}>>m^{2}$ limit)

$$
\begin{aligned}
\delta_{\text {vac }}+\delta_{\text {vertex }}+\delta_{R}=\frac{\alpha_{e m}}{\pi} & \left\{\ln \left(\frac{\left(\Delta E_{s}\right)^{2}}{E_{e} E_{e}^{\prime}}\right)\left[\ln \left(\frac{Q^{2}}{m^{2}}\right)-1\right]\right. \\
+ & \left.\frac{13}{6} \ln \left(\frac{Q^{2}}{m^{2}}\right)-\frac{28}{9}-\frac{1}{2} \ln ^{2}\left(\frac{E_{e}}{E_{e}^{\prime}}\right)-\frac{\pi^{2}}{6}+S p\left(\cos ^{2} \frac{\theta_{e}}{2}\right)\right\},
\end{aligned}
$$

where $\Delta E_{s}$, which is the maximum soft-photon energy in the c.m. system of (recoiling proton + soft-photon), is determined as in Eq. (A49), when applying this formula to the

\footnotetext{
${ }^{3}$ Note that an incorrect expression is used in 50 for the vacuum polarization contribution due to $\mu^{+} \mu^{-}$pairs (Eq. (A5) in their paper).
} 
scattered electron spectrum.

We can compare Eq. (A72) with the recent calculation of Maximon and Tjon [24], where this calculation was also performed (using a finite photon mass to regularize the IR divergences) without doing any approximations. Comparing Eq. (A72) with their $Z$-independent term ( $Z$ being the hadron charge) - i.e. when not considering radiative corrections at the proton side or two-photon exchange contributions at this point - we find exactly the same result. As was noted in Ref. [24], the last two terms of Eq. (A72) were omitted by Mo and Tsai 22].

We can approximately take into account the higher order radiative corrections by exponentiating the first order vertex and real radiative corrections. This is strictly true only for the IR divergent part of the vertex correction and soft photon emission contribution, and was demonstrated in Refs. [26,48] (see e.g. Refs. [51,52 for pedagogical derivations). The application of this exponentiation procedure also to the finite part consists of an approximation which can be checked by comparing the result with the first order formula of Eq. (A71). For the photon polarization contribution, we iterate the first order vacuum polarization contribution of Eq. (A69) to all orders (resumming all vacuum bubbles of the type of Fig. 19 (c)) by keeping the photon self-energy in the denominator as in Eq. (A67). Remark that a resummation of the first order vacuum polarization contribution does not lead to an exponentiated form. Assuming exponentiation for the finite parts of the vertex and soft photon emission contributions - as occurs for their IR divergent pieces - leads then to the radiative correction formula

$$
\left(\frac{d \sigma}{d \Omega_{e}^{\prime}}\right)_{V I R T U A L \gamma}+\left(\frac{d \sigma}{d \Omega_{e}^{\prime}}\right)_{R E A L S O F T \gamma}=\left(\frac{d \sigma}{d \Omega_{e}^{\prime}}\right)_{B O R N} \frac{e^{\delta_{\text {vertex }}+\delta_{R}}}{\left(1-\delta_{\text {vac }} / 2\right)^{2}} .
$$

\section{Radiative corrections at the proton side and two-photon exchange contributions}

In the previous sections, we considered radiative corrections to elastic electron scattering originating solely from the electron side (vertex correction and bremsstrahlung) and from the vacuum polarization. These corrections, which are the dominant ones, can be calculated model independently and follow from QED. To calculate the first order radiative corrections originating from the proton side (proton vertex correction, bremsstrahlung from proton and direct and crossed two-photon exchange contributions), one needs a model for the internal structure of the nucleon because one requires knowledge of off-shell (or half off-shell) $\gamma N N$ vertices. This model dependence will become important if one aims at a precision of electron scattering experiments at the $1 \%$ level. To quantify the magnitude of those effects, we refer to the recent work of Maximon and Tjon [24], where an initial study was performed of the size of internal structure effects.

In Ref. [24], the proton current was taken to have the usual on-shell form and form factors were included in the calculation. The calculation of Ref. 24] goes beyond previous works [21,22], as the proton vertex correction and the bremsstrahlung from the proton where calculated without approximations within the given model for the proton current. In the

calculation of the direct and crossed box diagrams (two-photon exchange contributions), a less drastic approximation was made in [24] as in [21] (where those box diagrams where only calculated in the soft-photon approximation). 
The calculation of Ref. [24 yields then the correction formula for elastic electron scattering :

$$
\left(\frac{d \sigma}{d \Omega_{e}^{\prime}}\right)_{T O T A L}=\left(\frac{d \sigma}{d \Omega_{e}^{\prime}}\right)_{B O R N}\left(1+\delta_{v a c}+\delta_{v e r t e x}+\delta_{R}+Z \delta_{1}+Z^{2}\left(\delta_{2}^{(0)}+\delta_{2}^{(1)}\right)\right.
$$

where $\delta_{v a c}, \delta_{\text {vertex }}$ and $\delta_{R}$ are given as above (Eq.(A72)). The terms in Eq. (A74) proportional to $Z$ (hadron charge) and $Z^{2}$ contain the corrections from the proton side. The correction $\delta_{1}$, proportional to $Z$, was calculated in Ref. [24] as

$$
\delta_{1}=\frac{2 \alpha_{e m}}{\pi}\left\{\ln \left(\frac{4\left(\Delta E_{s}\right)^{2}}{Q^{2} x}\right) \ln \eta+S p\left(1-\frac{\eta}{x}\right)-S p\left(1-\frac{1}{\eta x}\right)\right\}
$$

where $\Delta E_{s}$ and $\eta$ are given as in Eq. (A49) and where the variable $x$ is defined by

$$
x=\frac{(Q+\rho)^{2}}{4 M_{N}^{2}}, \quad \rho^{2}=Q^{2}+4 M_{N}^{2}
$$

The correction proportional to $Z^{2}$ was split into two parts in Ref. 24]. The contribution $\delta_{2}^{(0)}$, independent of the nucleon form factors was calculated in Ref. 24 as :

$$
\begin{aligned}
\delta_{2}^{(0)}= & \frac{\alpha_{e m}}{\pi}\left\{\ln \left(\frac{4\left(\Delta E_{s}\right)^{2}}{M_{N}^{2}}\right)\left(\frac{E_{N}^{\prime}}{\left|\vec{p}_{N}^{\prime}\right|} \ln x-1\right)+1\right. \\
+ & \left.\frac{E_{N}^{\prime}}{\left|\vec{p}_{N}^{\prime}\right|}\left(-\frac{1}{2} \ln ^{2} x-\ln x \ln \left(\frac{\rho^{2}}{M_{N}^{2}}\right)+\ln x-S p\left(1-\frac{1}{x^{2}}\right)+2 S p\left(-\frac{1}{x}\right)+\frac{\pi^{2}}{6}\right)\right\}
\end{aligned}
$$

where $\rho$ is defined as in Eq. (A76), and where $E_{N}^{\prime}\left(\left|\vec{p}_{N}^{\prime}\right|\right)$ are the lab energy (momentum) of the recoiling nucleon. For the lengthier expression of $\delta_{2}^{(1)}$, which depends on the nucleon form factors, we refer to Ref. [24]. 


\section{APPENDIX B: TREATMENT OF SINGULARITIES}

In the numerical calculation of the amplitudes for the virtual photon radiative corrections to the $e p \rightarrow e p \gamma$ reaction, we need to calculate two or three dimensional Feynman parameter integrals, as discussed in section $\amalg I I \mathrm{G}$. In the integration over the first variable, the numerator consists of polynomials and the denominators may have some structures of the form $\left(\alpha^{\prime} x+\beta^{\prime} \pm i \varepsilon^{\prime}\right)^{n}$, or $\left(\alpha^{\prime} x^{2}+\beta^{\prime} x+\gamma^{\prime} \pm i \varepsilon^{\prime}\right)^{n}$ with $n=1,2$. Therefore, in the calculations, the following integrals appear :

$$
\lim _{\varepsilon^{\prime} \rightarrow 0^{+}} \int_{a}^{b} \frac{x^{m} d x}{\left(\alpha^{\prime} x+\beta^{\prime} \pm i \varepsilon^{\prime}\right)^{n}} \quad \text { or } \quad \lim _{\varepsilon^{\prime} \rightarrow 0^{+}} \int_{a}^{b} \frac{x^{m} d x}{\left(\alpha^{\prime} x^{2}+\beta^{\prime} x+\gamma^{\prime} \pm i \varepsilon^{\prime}\right)^{n}} .
$$

When the denominator has no singularities in the integration range, it is, in principle, easy to calculate these integrals which have the form

$$
\int_{a}^{b} \frac{x^{m} d x}{\left(\alpha^{\prime} x+\beta^{\prime}\right)^{n}} \quad \text { or } \quad \int_{a}^{b} \frac{x^{m} d x}{\left(\alpha^{\prime} x^{2}+\beta^{\prime} x+\gamma^{\prime}\right)^{n}}
$$

Some recurrence relations for these integrals are known [53,54, but for small values of $\alpha^{\prime}$ as compared to $\beta^{\prime}$ or to $\gamma^{\prime}$, these relations are unstable numerically. In these cases, we have used either a Taylor expansion or the usual Gauss-Legendre integration method to get very accurate results.

In the following part of this appendix, we give the relations used when the denominators in Eq. (B1) have singularities in the integration range except in $a$ or $b$. The details are given elsewhere 28]. The principle of the method is based on the following relation :

$$
\begin{aligned}
\lim _{\varepsilon \rightarrow 0^{+}} \int_{a}^{b} \frac{x^{m} d x}{\left(x-x_{0} \pm i \varepsilon\right)^{n}} & \\
=\lim _{\varepsilon \rightarrow 0^{+}} \lim _{\eta \rightarrow 0^{+}}\left[\int_{a}^{x_{0}-\eta} \frac{x^{m} d x}{\left(x-x_{0} \pm i \varepsilon\right)^{n}}\right. & +\int_{x_{0}-\eta}^{x_{0}+\eta} \frac{x^{m} d x}{\left(x-x_{0} \pm i \varepsilon\right)^{n}} \\
& \left.+\int_{x_{0}+\eta}^{b} \frac{x^{m} d x}{\left(x-x_{0} \pm i \varepsilon\right)^{n}}\right] .
\end{aligned}
$$

Each integral can be separated in a real part and an imaginary part and we can use for them the analytical expressions given in [53].

Let us start with the case where the denominator is a polynomial of degree 1 in the integration variable. In that case, there is only one singularity for $x_{0}=-\beta^{\prime} / \alpha^{\prime}$ and the sign of the imaginary part will depend on the sign of $\alpha^{\prime}$. For $n=1$ and $\alpha^{\prime}>0$, we have

$$
\lim _{\varepsilon^{\prime} \rightarrow 0^{+}} \int_{a}^{b} \frac{x^{m} d x}{\alpha^{\prime} x+\beta^{\prime} \pm i \varepsilon^{\prime}}=\frac{1}{\alpha^{\prime}} \lim _{\varepsilon \rightarrow 0^{+}} \int_{a}^{b} \frac{x^{m} d x}{x-x_{0} \pm i \varepsilon}, \quad \varepsilon=\frac{\varepsilon^{\prime}}{\alpha^{\prime}} .
$$

When $\alpha^{\prime}<0$, we have only to replace $\pm i \varepsilon$ by $\mp i \varepsilon$ in the right hand side of the Eq. (B4). We now define the following quantities 


$$
\begin{aligned}
& J_{1}=\frac{1}{2} \log \frac{\left(b-x_{0}\right)^{2}}{\left(a-x_{0}\right)^{2}} \\
& J_{n}=\frac{1}{n-1}\left[\left(b-x_{0}\right)^{n-1}-\left(a-x_{0}\right)^{n-1}\right], \quad n \geq 2
\end{aligned}
$$

to obtain the relations

$$
\begin{aligned}
& \lim _{\varepsilon \rightarrow 0^{+}} \int_{a}^{b} \frac{d x}{x-x_{0} \pm i \varepsilon}=J_{1} \mp i \pi, \\
& \lim _{\varepsilon \rightarrow 0^{+}} \int_{a}^{b} \frac{x d x}{x-x_{0} \pm i \varepsilon}=J_{2}+x_{0} J_{1} \mp i \pi x_{0}, \\
& \lim _{\varepsilon \rightarrow 0^{+}} \int_{a}^{b} \frac{x^{2} d x}{x-x_{0} \pm i \varepsilon}=J_{3}+2 x_{0} J_{2}+x_{0}^{2} J_{1} \mp i \pi x_{0}^{2}, \\
& \lim _{\varepsilon \rightarrow 0^{+}} \int_{a}^{b} \frac{x^{3} d x}{x-x_{0} \pm i \varepsilon}=J_{4}+3 x_{0} J_{3}+3 x_{0}^{2} J_{2}+x_{0}^{3} J_{1} \mp i \pi x_{0}^{3}, \\
& \lim _{\varepsilon \rightarrow 0^{+}} \int_{a}^{b} \frac{x^{4} d x}{x-x_{0} \pm i \varepsilon}=J_{5}+4 x_{0} J_{4}+6 x_{0}^{2} J_{3}+4 x_{0}^{3} J_{2}+x_{0}^{4} J_{1} \mp i \pi x_{0}^{4} . \\
& \ldots
\end{aligned}
$$

For $n=2$ and $\alpha^{\prime}>0$, we have

$$
\lim _{\varepsilon^{\prime} \rightarrow 0^{+}} \int_{a}^{b} \frac{x^{m} d x}{\left(\alpha^{\prime} x+\beta^{\prime} \pm i \varepsilon^{\prime}\right)^{2}}=\frac{1}{\alpha^{\prime 2}} \lim _{\varepsilon \rightarrow 0^{+}} \int_{a}^{b} \frac{x^{m} d x}{\left(x-x_{0} \pm i \varepsilon\right)^{2}} .
$$

When $\alpha^{\prime}<0$, we have only to replace $\pm i \varepsilon$ by $\mp i \varepsilon$ in the right hand side of the Eq. (B11). We next define the following quantities :

$$
\begin{aligned}
I_{0} & =\frac{1}{a-x_{0}}-\frac{1}{b-x_{0}}, \\
I_{1} & =\frac{1}{2} \log \frac{\left(b-x_{0}\right)^{2}}{\left(a-x_{0}\right)^{2}}, \\
I_{n} & =\frac{1}{n-1}\left[\left(b-x_{0}\right)^{n-1}-\left(a-x_{0}\right)^{n-1}\right], \quad n \geq 2 .
\end{aligned}
$$

In terms of these quantities, the integrals of Eq. (B1) with $n=2$ are given by

$$
\begin{aligned}
& \lim _{\varepsilon \rightarrow 0^{+}} \int_{a}^{b} \frac{d x}{\left(x-x_{0} \pm i \varepsilon\right)^{2}}=I_{0}, \\
& \lim _{\varepsilon \rightarrow 0^{+}} \int_{a}^{b} \frac{x d x}{\left(x-x_{0} \pm i \varepsilon\right)^{2}}=I_{1}+x_{0} I_{0} \mp i \pi, \\
& \lim _{\varepsilon \rightarrow 0^{+}} \int_{a}^{b} \frac{x^{2} d x}{\left(x-x_{0} \pm i \varepsilon\right)^{2}}=I_{2}+2 x_{0} I_{1}+x_{0}^{2} I_{0} \mp i 2 \pi x_{0}, \\
& \lim _{\varepsilon \rightarrow 0^{+}} \int_{a}^{b} \frac{x^{3} d x}{\left(x-x_{0} \pm i \varepsilon\right)^{2}}=I_{3}+3 x_{0} I_{2}+3 x_{0}^{2} I_{1}+x_{0}^{3} I_{0} \mp i 3 \pi x_{0}^{2},
\end{aligned}
$$


We can notice that the real part of these integrals for $n=1$ as well as for $n=2$ can be derived from the binomial expansion $\left(x_{0}+X\right)^{m}$. In the case of $n=1$, the imaginary part is proportional to $\pi f\left(x_{0}\right)$ where $f(x)$ is the numerator of the integrand. For $n=2$, it is straightforward to show [28] that the imaginary part is proportional to $\pi f^{\prime}\left(x_{0}\right)$.

When the form of the denominator is $\left(\alpha^{\prime} x^{2}+\beta^{\prime} x+\gamma^{\prime} \pm i \varepsilon^{\prime}\right)^{n}$, i.e. a polynomial of degree 2 in the integration variable, it is always possible to come back to the preceding cases. When $\alpha^{\prime}>0$, we have

$$
\lim _{\varepsilon^{\prime} \rightarrow 0^{+}} \int_{a}^{b} \frac{x^{m} d x}{\left(\alpha^{\prime} x^{2}+\beta^{\prime} x+\gamma^{\prime} \pm i \varepsilon^{\prime}\right)^{n}}=\frac{1}{\alpha^{\prime n}} \lim _{\varepsilon \rightarrow 0^{+}} \int_{a}^{b} \frac{x^{m} d x}{\left(x^{2}+\beta x+\gamma \pm i \varepsilon\right)^{n}},
$$

with the following definitions :

$$
\beta=\frac{\beta^{\prime}}{\alpha^{\prime}}, \quad \gamma=\frac{\gamma^{\prime}}{\alpha^{\prime}}, \quad \varepsilon=\frac{\varepsilon^{\prime}}{\alpha^{\prime}} .
$$

The integrand in Eq. (B19) has some singularities when $\delta=\beta^{2}-4 \gamma$ is positive. When $\alpha^{\prime}<0$, we have only to replace $\pm i \varepsilon$ by $\mp i \varepsilon$ on the right hand side of Eq. (B19).

It can be shown [28] that

$$
\lim _{\varepsilon \rightarrow 0^{+}} \int_{a}^{b} \frac{x^{m} d x}{\left(x^{2}+\beta x+\gamma \pm i \varepsilon\right)^{n}}=\lim _{\tilde{\varepsilon} \rightarrow 0^{+}} \int_{a}^{b} \frac{x^{m} d x}{\left(x-x_{+}^{R}+i \tilde{\varepsilon}\right)^{n}\left(x-x_{-}^{R}-i \tilde{\varepsilon}\right)^{n}},
$$

with the definitions

$$
x_{+}^{R}=\frac{-\beta+\sqrt{\delta}}{2}, \quad x_{-}^{R}=\frac{-\beta-\sqrt{\delta}}{2}, \quad \tilde{\varepsilon}=\frac{2 \varepsilon}{\delta} .
$$

These integrals can be easily calculated using the decomposition of the fraction into elementary fractions. For $n=1$, we obtain

$$
\begin{aligned}
& \lim _{\varepsilon \rightarrow 0^{+}} \int_{a}^{b} \frac{x^{m} d x}{x^{2}+\beta x+\gamma \pm i \varepsilon} \\
& =\frac{1}{\sqrt{\delta}} \lim _{\tilde{\varepsilon} \rightarrow 0^{+}} \int_{a}^{b} \frac{x^{m} d x}{x-x_{+}^{R} \pm i \tilde{\varepsilon}}-\frac{1}{\sqrt{\delta}} \lim _{\tilde{\varepsilon} \rightarrow 0^{+}} \int_{a}^{b} \frac{x^{m} d x}{x-x_{-}^{R} \mp i \tilde{\varepsilon}},
\end{aligned}
$$

and for $n=2$

$$
\begin{aligned}
& \lim _{\varepsilon \rightarrow 0^{+}} \int_{a}^{b} \frac{x^{m} d x}{\left(x^{2}+\beta x+\gamma \pm i \varepsilon\right)^{2}} \\
& =\frac{1}{\delta} \lim _{\tilde{\varepsilon} \rightarrow 0^{+}} \int_{a}^{b} \frac{x^{m} d x}{\left(x-x_{+}^{R} \pm i \tilde{\varepsilon}\right)^{2}}-\frac{2}{\delta^{3 / 2}} \lim _{\tilde{\varepsilon} \rightarrow 0^{+}} \int_{a}^{b} \frac{x^{m} d x}{x-x_{+}^{R} \pm i \tilde{\varepsilon}} \\
& \quad+\frac{1}{\delta} \lim _{\tilde{\varepsilon} \rightarrow 0^{+}} \int_{a}^{b} \frac{x^{m} d x}{\left(x-x_{-}^{R} \mp i \tilde{\varepsilon}\right)^{2}}+\frac{2}{\delta^{3 / 2}} \lim _{\tilde{\varepsilon} \rightarrow 0^{+}} \int_{a}^{b} \frac{x^{m} d x}{x-x_{-}^{R} \mp i \tilde{\varepsilon}} .
\end{aligned}
$$




\section{REFERENCES}

[1] Proceedings of the workshop VCS96, Ed. V. Breton, Clermont-Ferrand, 1996.

[2] P.A.M. Guichon and M. Vanderhaeghen, Prog. Part. Nucl. Phys. 41, 125 (1998).

[3] P.A.M. Guichon, G.Q. Liu and A.W. Thomas, Nucl. Phys. A591, 606 (1995).

[4] D. Drechsel, G. Knöchlein, A.Yu. Korchin, A. Metz and S. Scherer, Phys. Rev. C 57, 941 (1998).

[5] Marc Vanderhaeghen, Phys. Lett. B 368, 13 (1996).

[6] A. Metz and D. Drechsel, Z. Phys. A356, 351 (1996); Z. Phys. A359, 165 (1997).

[7] Th.R. Hemmert and B.R. Holstein, G. Knöchlein and S. Scherer, Phys. Rev. D 55, 2630 (1997); Phys. Rev. Lett. 79, 22 (1997).

[8] B. Pasquini and G. Salmè, Phys. Rev. C 57, 2589 (1998).

[9] Th.R. Hemmert, B.R. Holstein, G. Knöchlein and D. Drechsel, hep-ph/9910036.

[10] J.F.J. van den Brand et al., Phys. Rev. D 52, 4868 (1995).

[11] J. Roche et al., to be submitted for publication to Phys. Rev. Lett.

[12] JLab experiment E-93-050, spokespersons P.Y. Bertin, P.A.M. Guichon and C. HydeWright.

[13] MIT-Bates experiment, spokespersons J. Shaw and R. Miskimen.

[14] X. Ji, Phys. Rev. Lett. 78, 610 (1997).

[15] A.V. Radyushkin, Phys. Lett. B 380, 417 (1996).

[16] X. Ji, J. Phys. G 24, 1181 (1998).

[17] P.Y. Bertin, C. Hyde-Wright and Y. Roblin, Proceedings of the conference "Nuclear and Particle Physics with CEBAF at JLAB", Dubrovnik, 1998. Published in Fyzika B, vol. 8, 207 (1999).

[18] D. Ryckbosch, private communication.

[19] N. d'Hose et al., Letter of Intent for COMPASS experiment, 1999.

[20] J. Schwinger, Phys. Rev. 76, 790 (1949).

[21] Y.S. Tsai, Phys. Rev. 122, 1898 (1961).

[22] L.W. Mo, Y.S. Tsai, Rev. Mod. Phys. 41, 205 (1969).

[23] L.C. Maximon, Rev. Mod. Phys. 41, 193 (1969).

[24] L.C. Maximon and J.A. Tjon, in preparation; private communication.

[25] J.D. Bjorken and S.D. Drell, Relativistic quantum mechanics, McGraw-Hill, New York (1964).

[26] F. Bloch and A. Nordsieck, Phys. Rev. 52, 54 (1937).

[27] J.M. Jauch and F. Rohrlich, Theory of photons and electrons, Addison-Wesley Reading, Mass., 1955.

[28] J. Van de Wiele, D. Lhuillier, D. Marchand and M. Vanderhaeghen, preprint IPNODRE-97-30.

[29] D. Lhuillier, Ph.D. thesis, Caen, DAPNIA/SPhN-97-01T, (1997).

[30] D. Marchand, Ph.D. thesis, Clermont-Ferrand, DAPNIA/SPhN-98-04T, (1998).

[31] L.C. Maximon and D.B. Isabelle, Phys. Rev. 133, B1344 (1964).

[32] L.C. Maximon and D.B. Isabelle, Phys. Rev. 136, B674 (1964).

[33] L.C. Maximon and S.E. Williamson, Nucl. Instrum. Meth. A258, 95 (1987).

[34] A.A. Akhundov, D.Yu. Bardin, and N.M. Shumeiko, Yad. Fiz. 44, 1517 (1986) [Sov. J. Nucl. Phys. 44, 988 (1986)].

[35] L.I. Schiff, Phys. Rev. 87, 750 (1952). 
[36] J.M. Friedrich, Ph.D. thesis, University Mainz, in preparation.

[37] L. Van Hoorebeke et al., in preparation.

[38] Marc Vanderhaeghen, Phys. Lett. B 402, 243 (1997).

[39] N. d'Hose et al., Letter of Intent for MAMI experiment, 1999.

[40] M. Vanderhaeghen, P.A.M. Guichon and M. Guidal, Phys. Rev. Lett. 80, 5064 (1998).

[41] M. Vanderhaeghen, P.A.M. Guichon and M. Guidal, Phys. Rev. D 61, 094017 (1999).

[42] T.-P. Cheng and L.-F. Li, Gauge theory of elementary particle interactions, Clarendon Press, Oxford, 1984.

[43] R. Gastmans and R. Meuldermans, Nucl. Phys. B63, 277 (1973).

[44] W.J. Marciano and A. Sirlin, Nucl. Phys. B88, 86 (1975).

[45] J. Schwinger, Phys. Rev. 73, 416L (1948).

[46] A.I. Akhiezer and V.B. Berestetskii, Quantum Electrodynamics, John Wiley \& Sons, New York, 1965.

[47] C. De Calan, H. Navelet and J. Picard, Nucl. Phys. B348, 47 (1991); note CEA-N-2624 (1990).

[48] D.R. Yennie, S.C. Frautschi and H. Suura, Ann. Phys. 13, 379 (1961).

[49] K. Mork and H. Olsen, Phys. Rev. 140, B1661 (1965).

[50] R.C. Walker et al., Phys. Rev. D 49, 5671 (1994).

[51] S. Weinberg, The Quantum Theory of Fields, Vol. I, Cambridge University Press, Cambridge, 1995.

[52] T. Muta, Foundations of Quantum Chromodynamics, World Scientific, Singapore, 1987.

[53] H.B. Dwight, Tables of integrals and other mathematical data, Macmillan, New York, 1961.

[54] I. S. Gradshteyn and I. M. Ryzhik, Table of Integrals, Series, and products, Academic Press, 1994. 


\section{TABLES}

TABLE I. Radiative corrections to elastic electron-proton scattering for MAMI and JLab kinematics. First column : $E_{e}$ in $\mathrm{GeV}$, second column : $\theta_{e}$ in deg, third column : $Q^{2}$ in $\mathrm{GeV}^{2}$. See text for details on the different contributions. The real radiative corrections are calculated with $\left(E_{e}^{\prime e l}-E_{e}^{\prime}\right)=0.01 E_{e}$. The total radiative correction (to first order) is indicated by $\delta_{t o t}$, and the exponentiated (EXP) result (except for the vacuum polarization contribution, see text) is shown in the last column.

\begin{tabular}{llllllll|ll}
\hline \hline & & & & & & & & \\
$E_{e}$ & $\theta_{e}$ & $Q^{2}$ & $\delta_{\text {vertex }}$ & $\delta_{\text {vacpol }}$ & $\delta_{R}$ & $\delta_{1}$ & $\delta_{2}^{(0)}$ & $\delta_{\text {tot }}$ & $\mathrm{EXP}$ \\
\hline & & & & & & & & \\
0.705 & 40.66 & 0.203 & -0.1673 & 0.0208 & -0.0453 & -0.0067 & -0.0018 & -0.2003 & -0.2025 \\
0.855 & 52.18 & 0.418 & -0.1881 & 0.0228 & -0.0245 & -0.0123 & -0.0034 & -0.2054 & -0.2087 \\
& & & & & & & & & \\
4.000 & 15.43 & 1.000 & -0.2149 & 0.0254 & -0.0260 & -0.0046 & -0.0055 & -0.2255 & -0.2277 \\
4.000 & 23.82 & 2.000 & -0.2374 & 0.0275 & 0.0018 & -0.0107 & -0.0096 & -0.2285 & -0.2322 \\
4.000 & 32.45 & 3.000 & -0.2511 & 0.0287 & 0.0300 & -0.0180 & -0.0128 & -0.2232 & -0.2292 \\
4.000 & 42.91 & 4.000 & -0.2611 & 0.0296 & 0.0623 & -0.0265 & -0.0150 & -0.2106 & -0.2200 \\
& & & & & & & & & \\
6.000 & 14.93 & 2.000 & -0.2374 & 0.0275 & -0.0097 & -0.0062 & -0.0089 & -0.2348 & -0.2371 \\
6.000 & 19.40 & 3.000 & -0.2511 & 0.0287 & 0.0092 & -0.0103 & -0.0121 & -0.2355 & -0.2390 \\
6.000 & 23.96 & 4.000 & -0.2611 & 0.0296 & 0.0284 & -0.0149 & -0.0146 & -0.2326 & -0.2376 \\
6.000 & 28.95 & 5.000 & -0.2689 & 0.0303 & 0.0490 & -0.0200 & -0.0166 & -0.2263 & -0.2334 \\
6.000 & 34.76 & 6.000 & -0.2754 & 0.0308 & 0.0718 & -0.0257 & -0.0181 & -0.2165 & -0.2261 \\
& & & & & & & & \\
\hline \hline
\end{tabular}




\section{FIGURES}

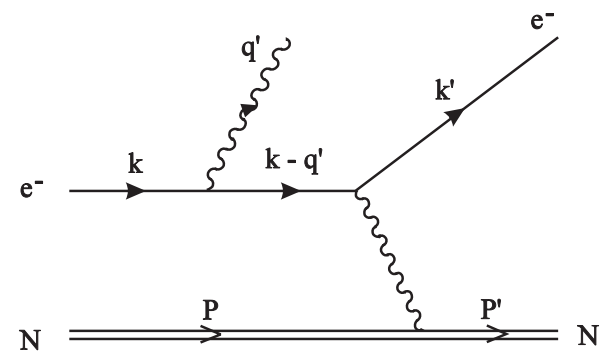

( $\mathrm{BH}$ i )

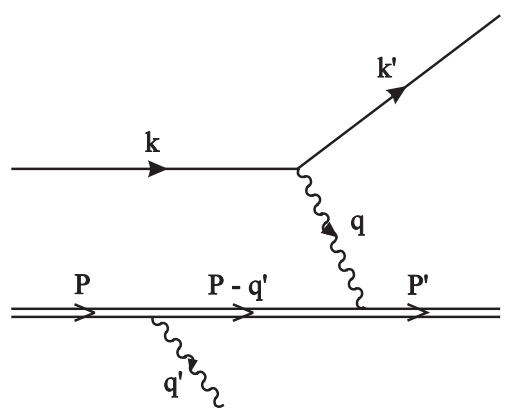

( BORN i )

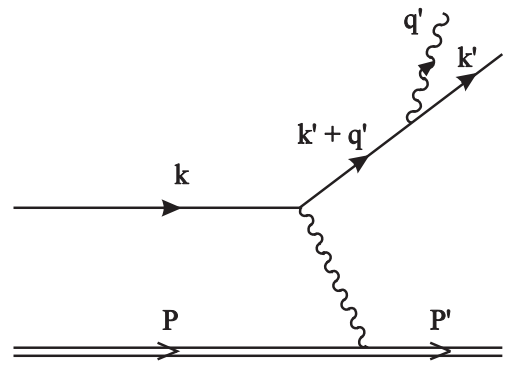

( $\mathrm{BH} \mathrm{f}$ )

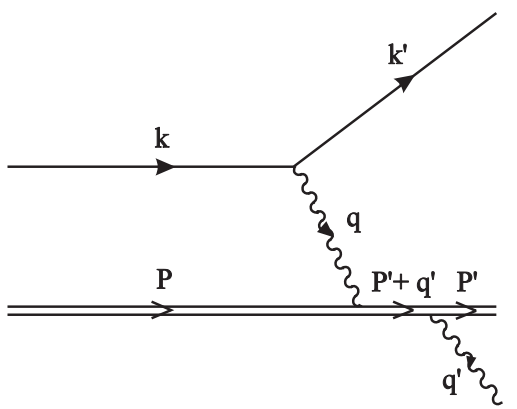

(BORN f)

FIG. 1. Tree level contributions to the $e p \rightarrow e p \gamma$ reaction: Bethe-Heitler diagrams (a) and (b); nucleon Born diagrams (c) and (d). 


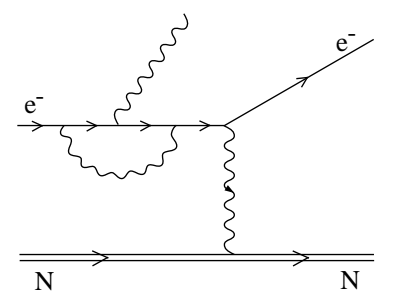

(V1i)

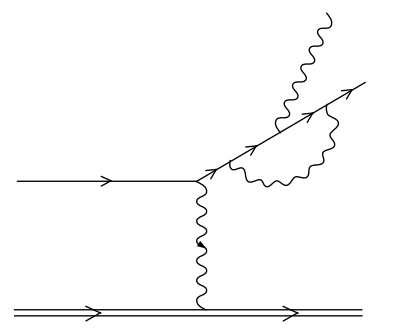

(V1f)

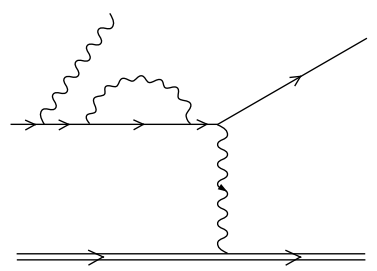

(Si)

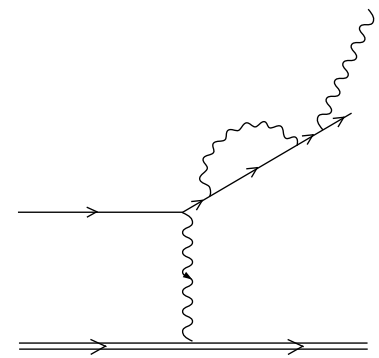

(Sf)

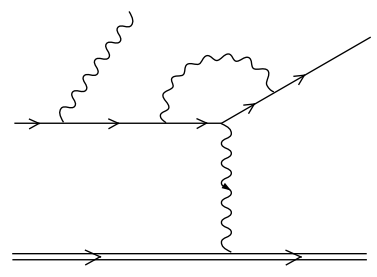

(V2i)

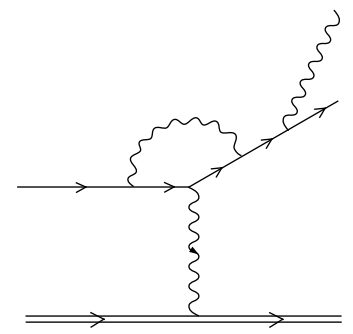

(V2f)

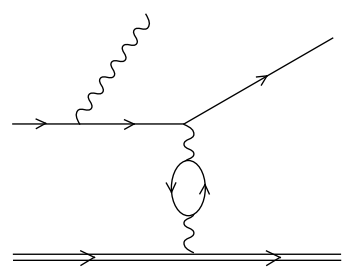

(P1i)

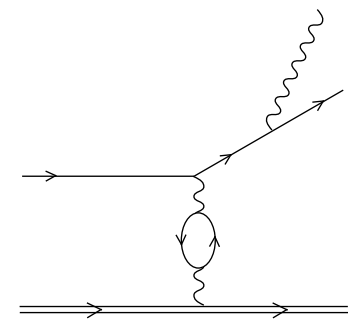

(P1f)

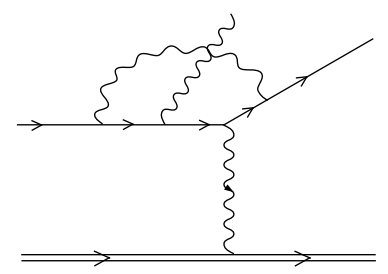

(V3i)

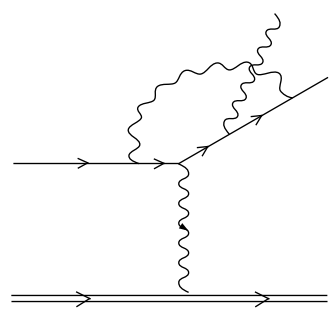

(V3f)
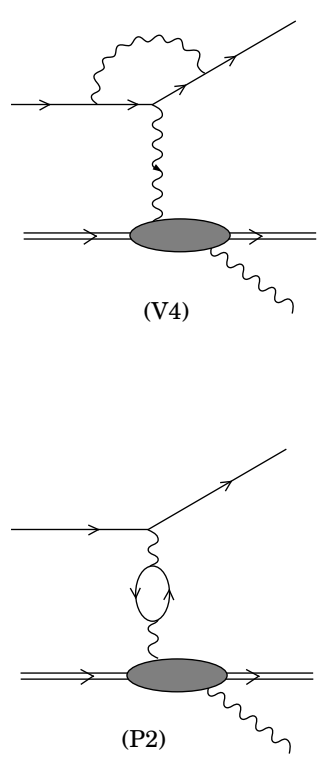

FIG. 2. First order virtual photon radiative corrections to the $e p \rightarrow e p \gamma$ reaction. 


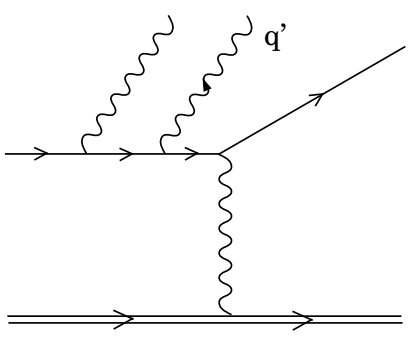

(b1i)

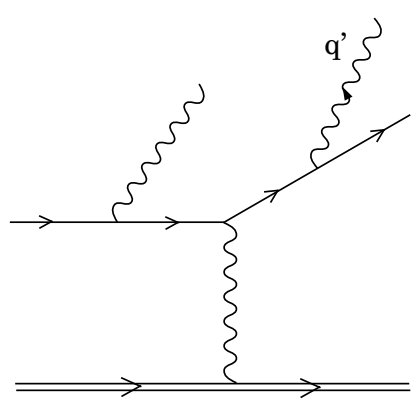

(b1f)

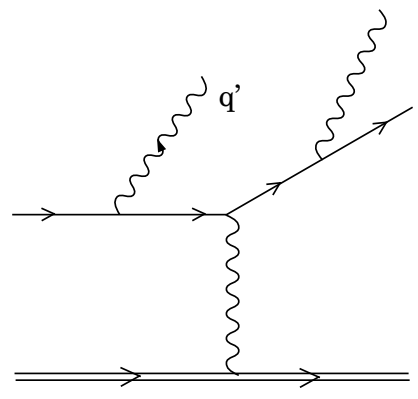

(b2i)

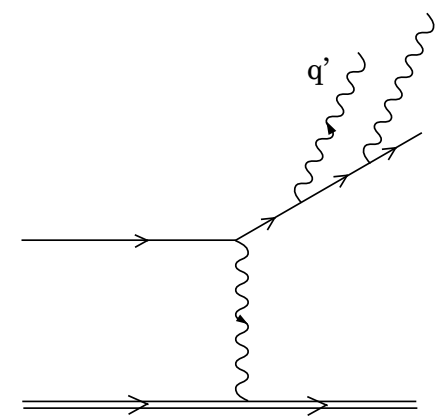

(b2f)

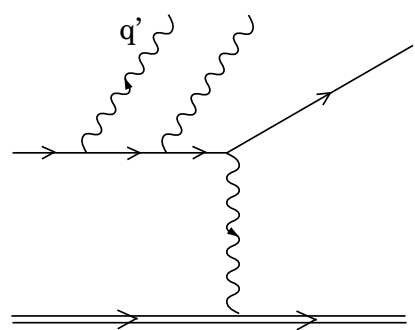

(b3i)

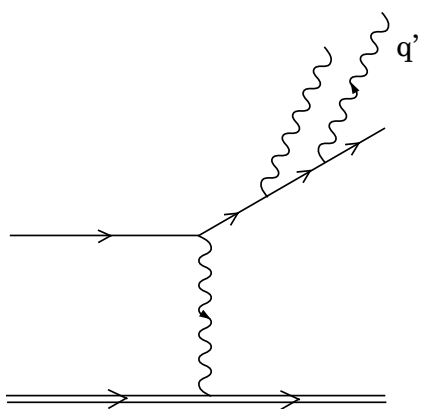

(b3f)

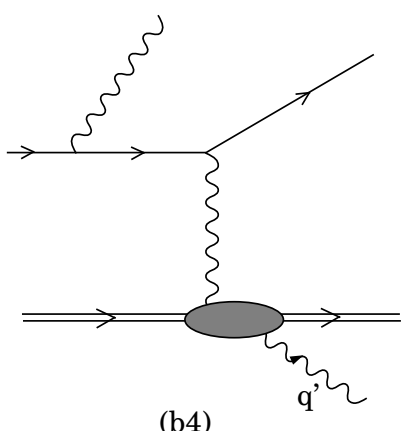

(b4)

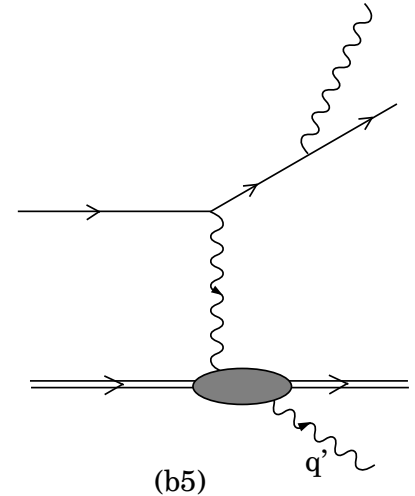

(b5)

FIG. 3. First order soft photon emission contributions to the $e p \rightarrow e p \gamma$ reaction. 


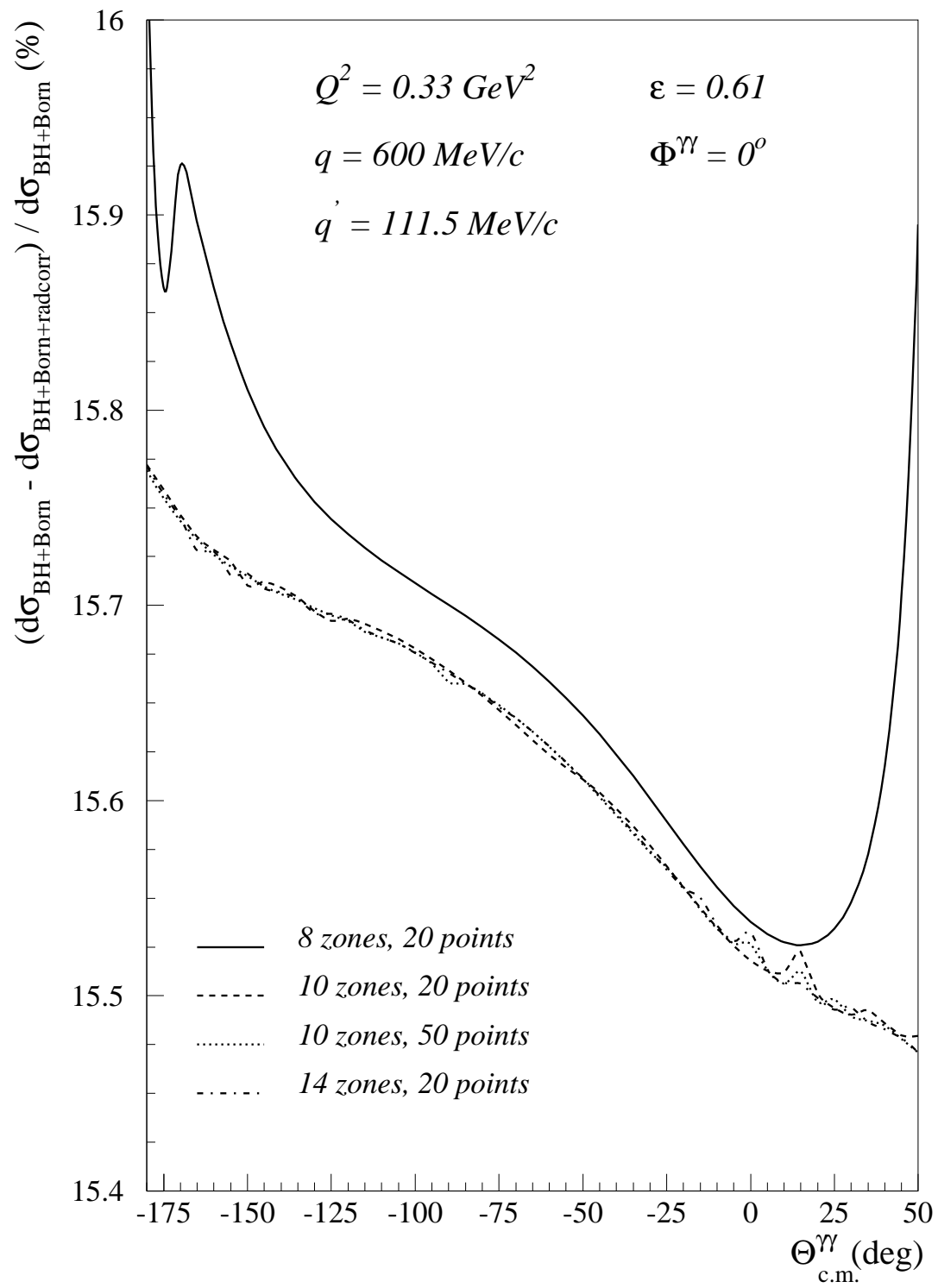

FIG. 4. Test of convergence : The relative effect of the virtual radiative corrections to the $\mathrm{BH}+$ Born cross section is shown as a function of the angle between the two photons $\left(q, q^{\prime}\right)$ for a typical MAMI kinematics. The curves correspond to tests performed with different densities of integration zones and points near the edge of the integration domain. They show the good numerical convergence obtained. 


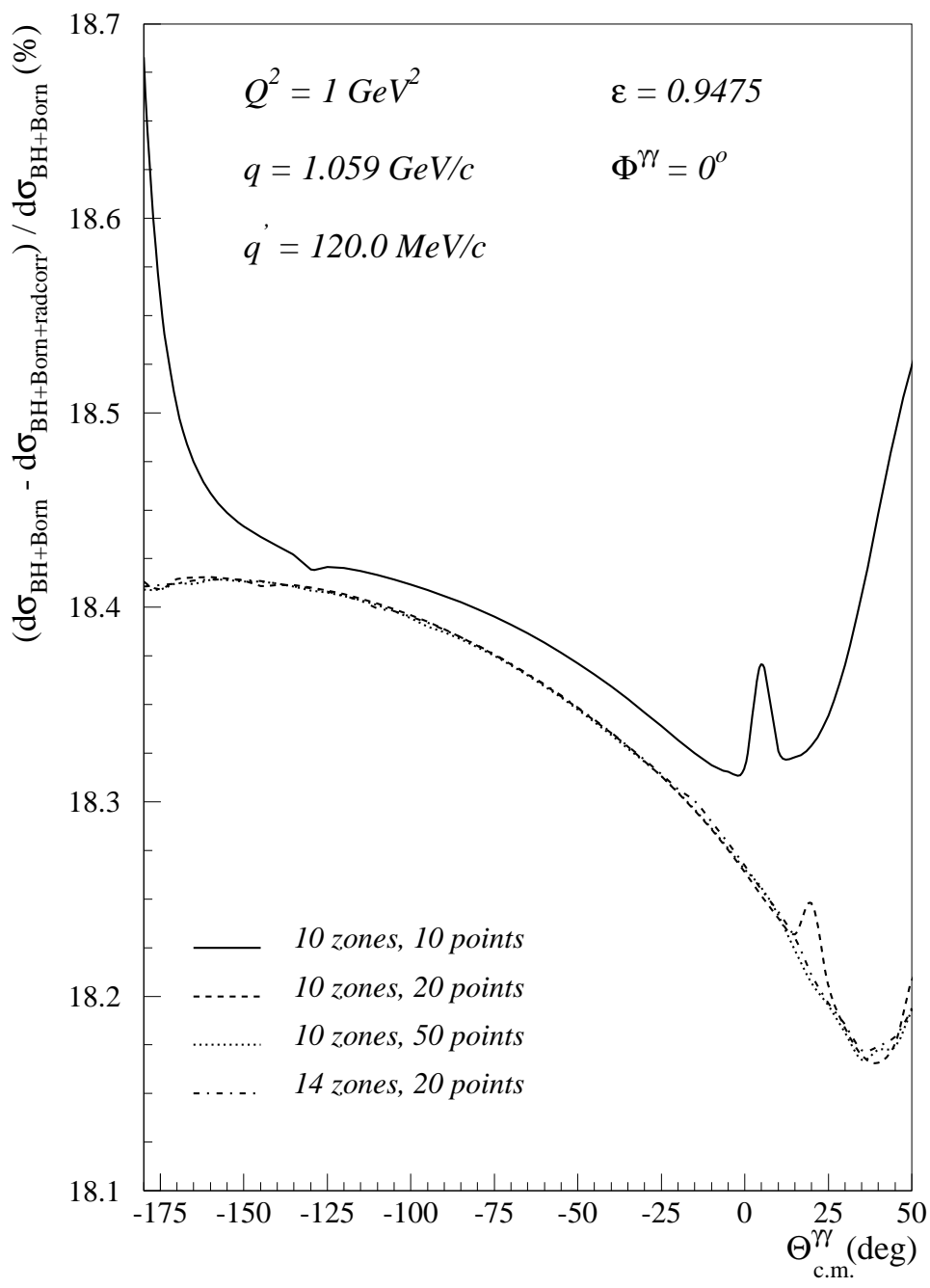

FIG. 5. Analogous test of convergence as in Fig. 4 but for JLab kinematics. 


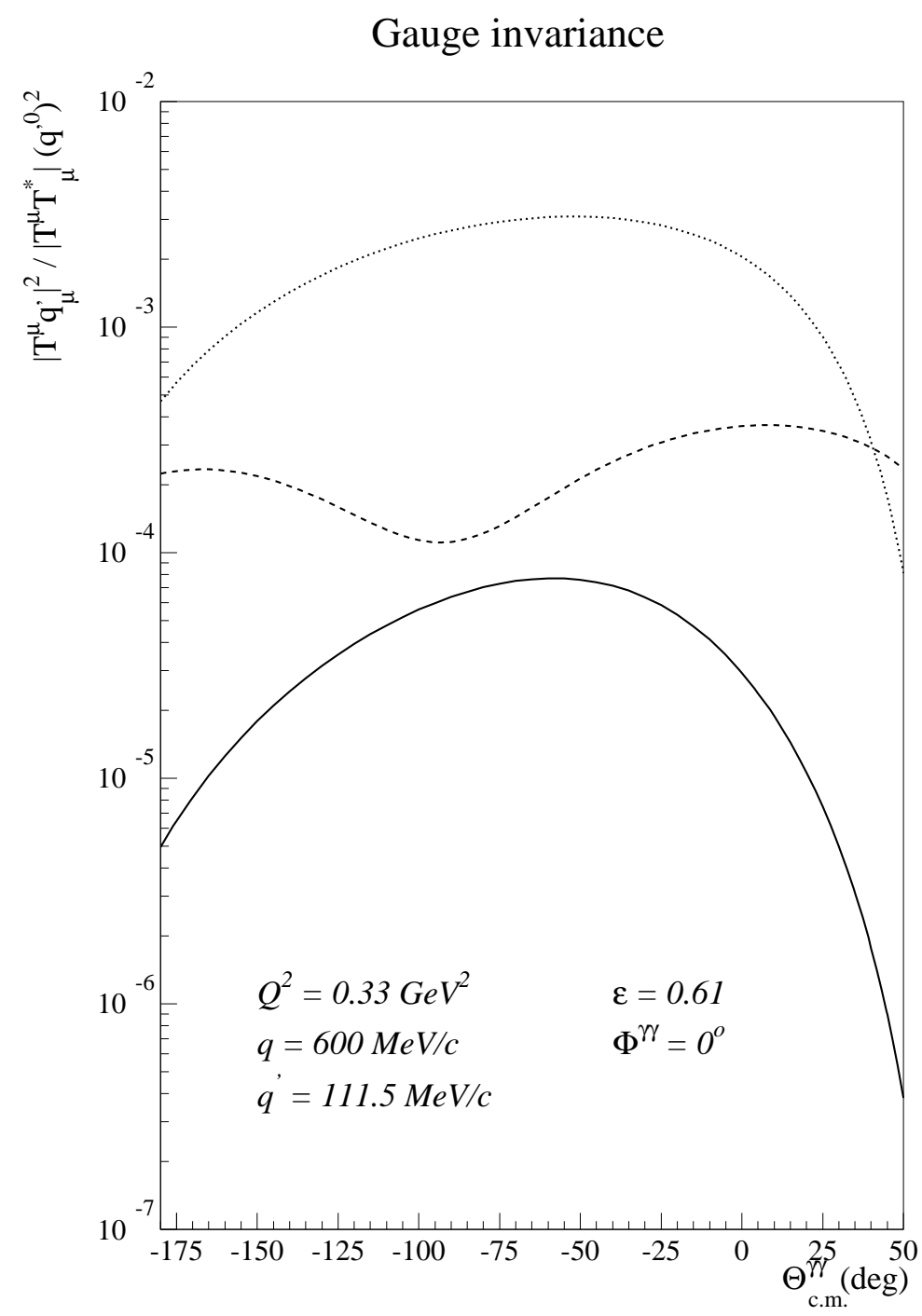

FIG. 6. Test of gauge invariance for MAMI kinematics. The dotted curve shows (for illustrative purpose only) the result when only the diagrams (V2i) and (V2f) of Fig. 2 are included. The dashed curve is the result of all analytically calculated virtual radiative corrections. The full curves show the result when also the numerical contributions (Feynman parameter integrals) are included. 


\section{Evolution in mass}

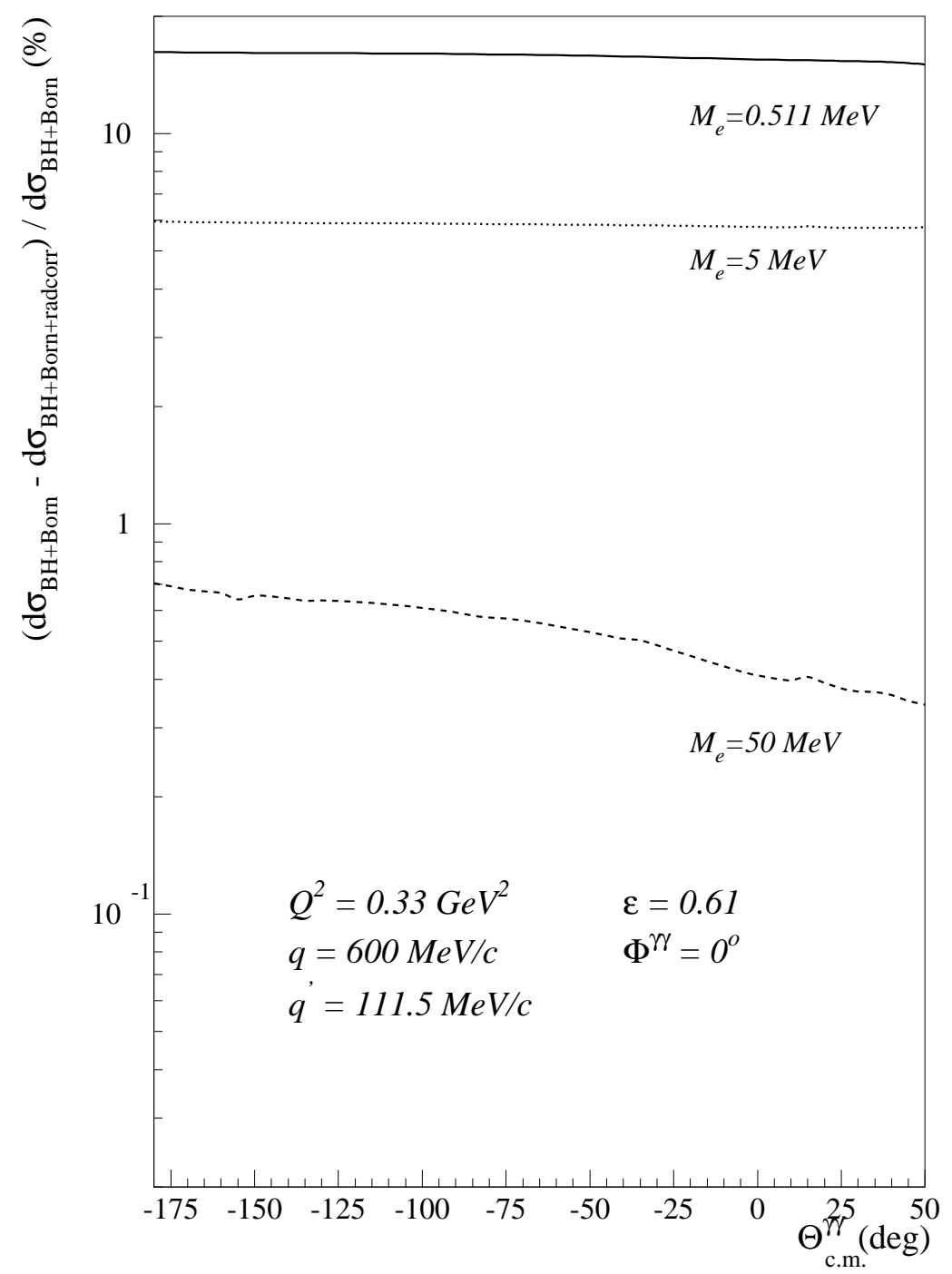

FIG. 7. Mass dependence of the virtual radiative corrections for MAMI kinematics. 


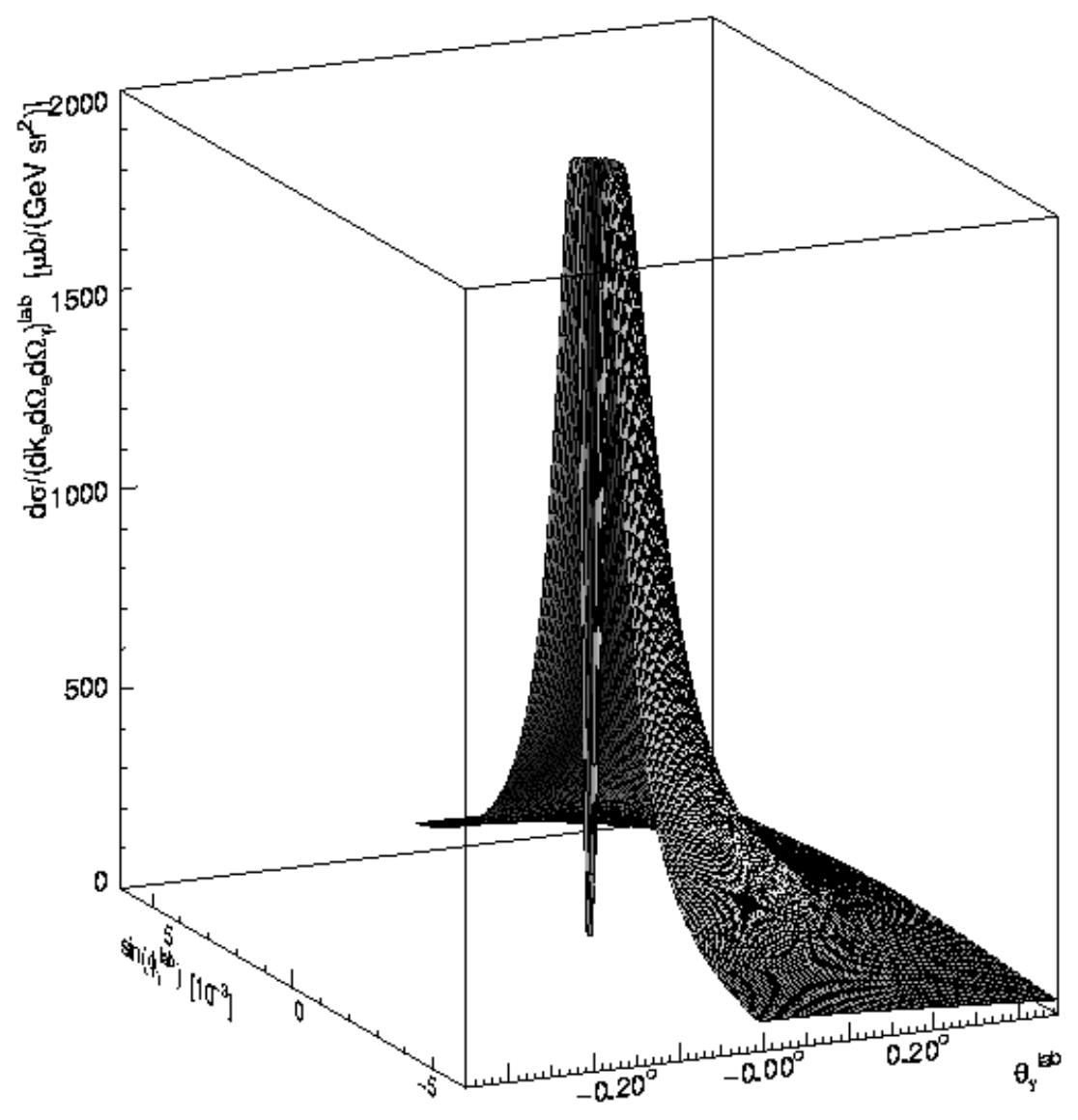

FIG. 8. Detail of the cross section for photon emission from an electron (Bethe-Heitler cross section), when the photon is emitted around the incoming electron direction. The electron kinematics correspond with : $E_{e}=855.0 \mathrm{MeV}, E_{e}^{\prime}=621.4 \mathrm{MeV}, \theta_{e}=52.18^{\circ}$. 

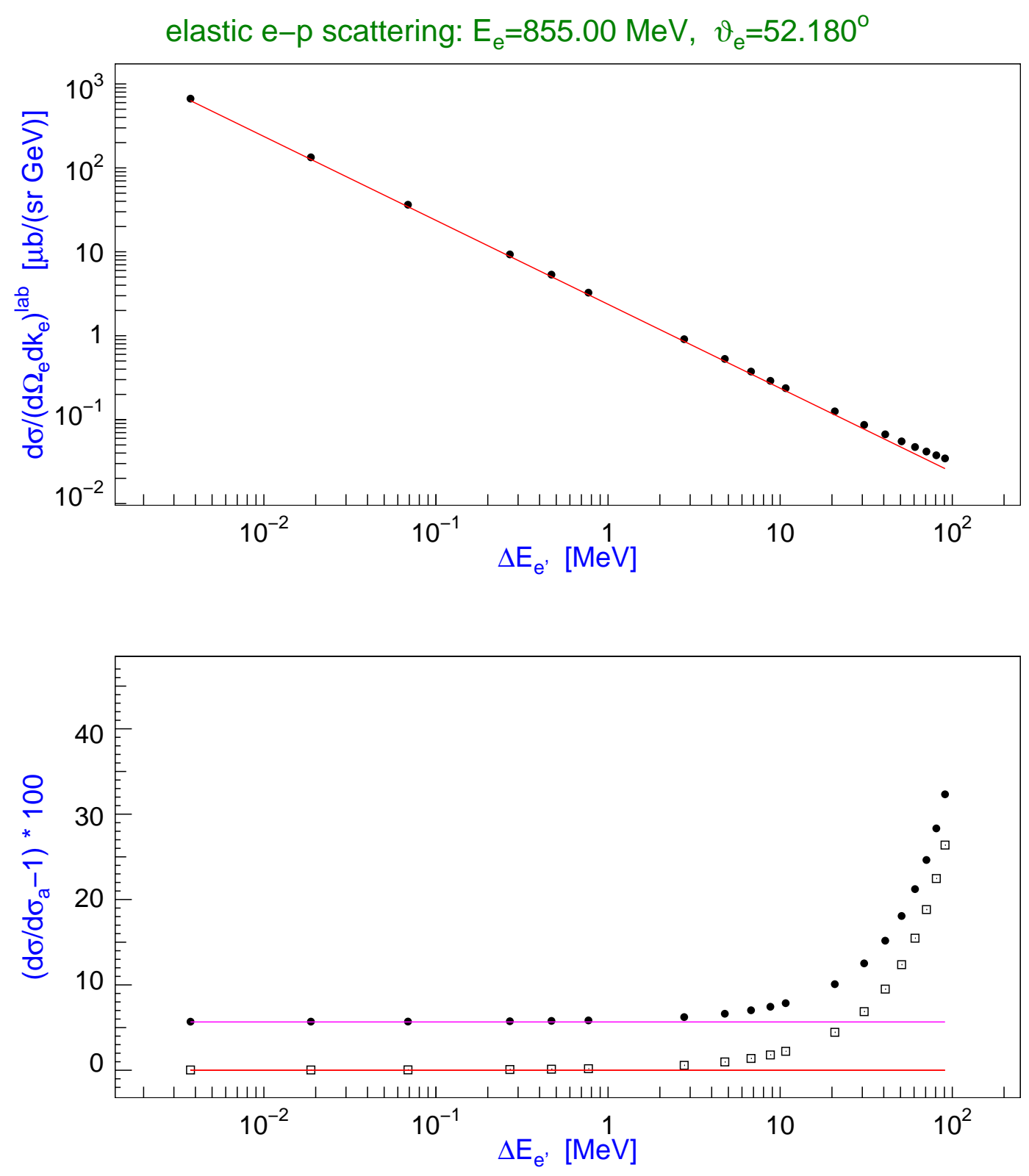

FIG. 9. Radiative tail of elastic electron-proton scattering. Upper plot : fully numerical calculation (black points) compared with the $1 / \Delta E_{e}^{\prime}$ dependence of the soft photon result (straight line). Lower plot : deviation between the full calculation, when only radiation from the electron is included (open diamonds) and when both radiation from electron and proton are taken into account (black points), with the soft photon result (straight lines). See text for details. 


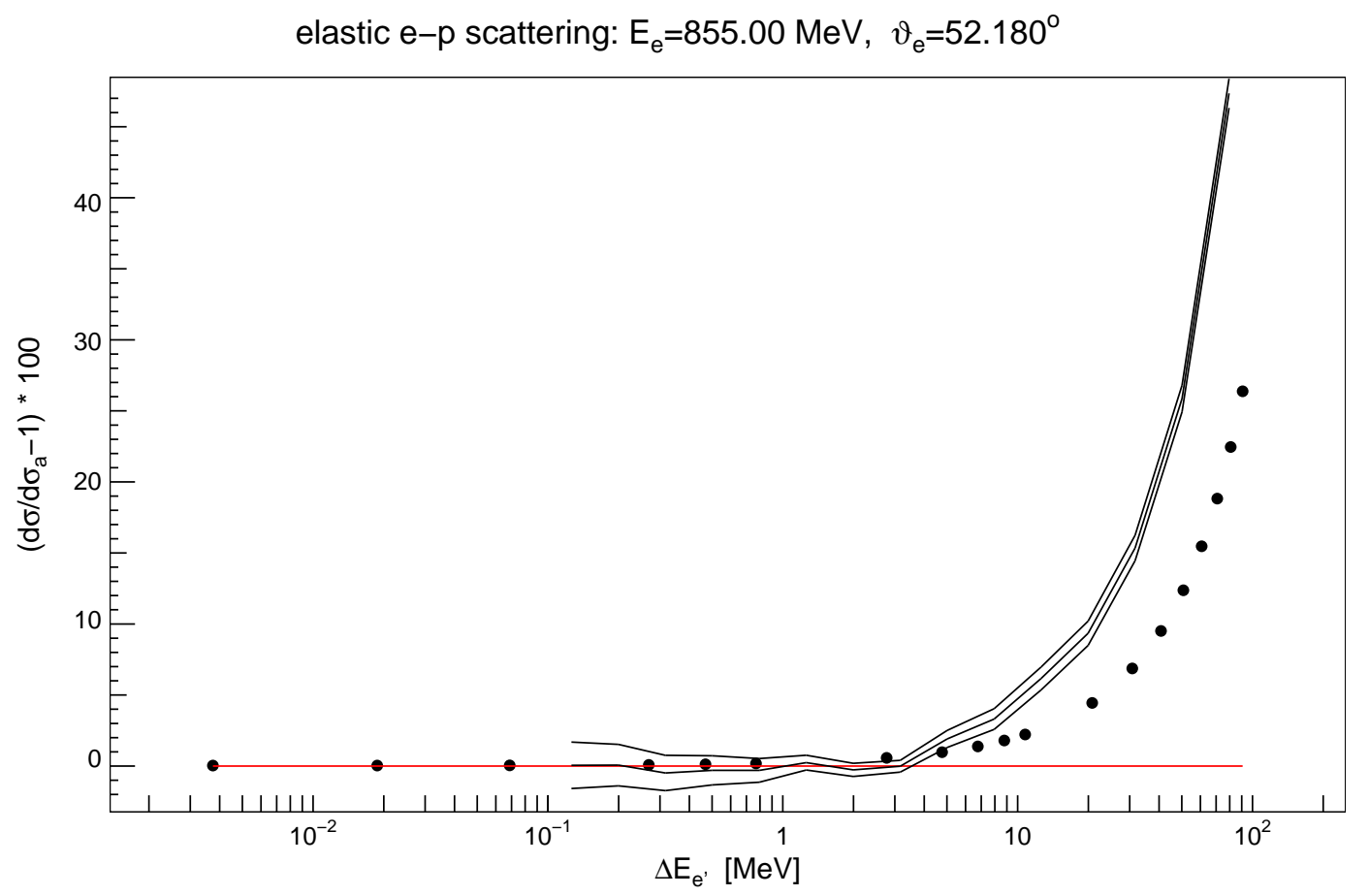

FIG. 10. Radiative tail of elastic electron-proton scattering at $E_{e}=855.00 \mathrm{MeV}$ and $\theta_{e}=$ $52.18^{\circ}$. A comparison is shown between fully numerical calculation (indicated by the points) and the simulation (curves, see text).

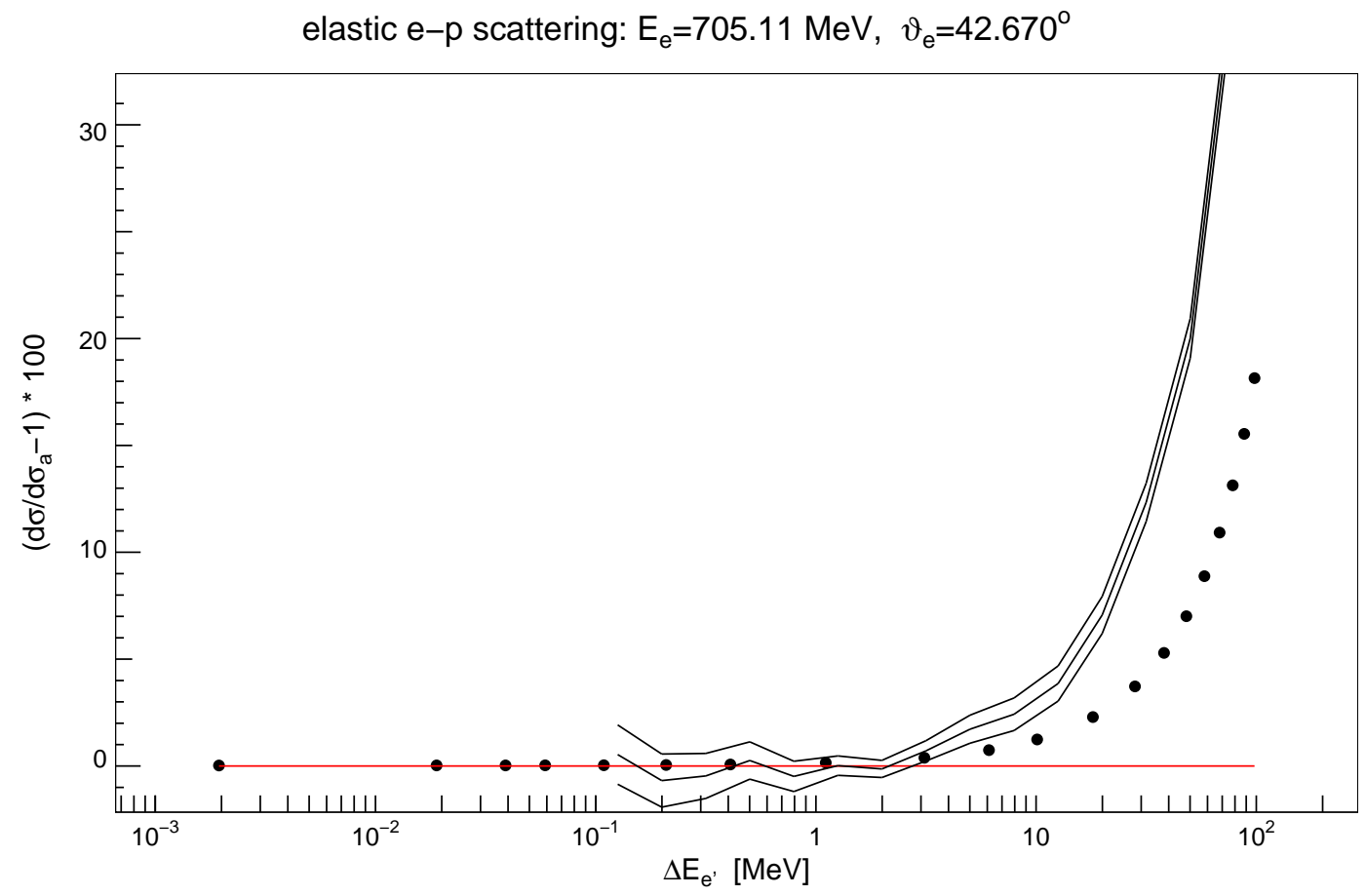

FIG. 11. Same as for Fig. 10, but for elastic electron-proton scattering at $E_{e}=705.11 \mathrm{MeV}$ and $\theta_{e}=42.67^{\circ}$. 

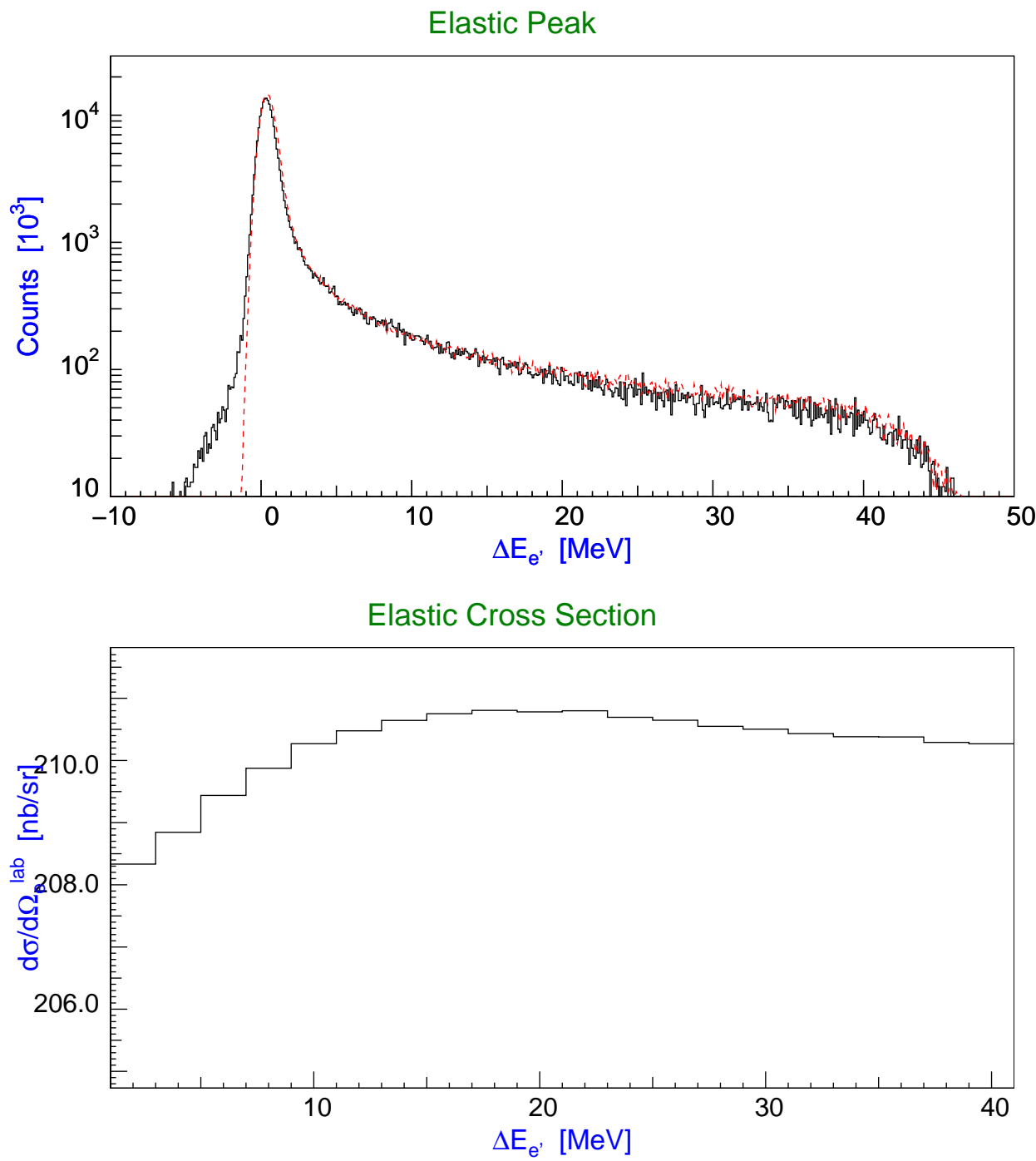

FIG. 12. Determination of the elastic cross section for the kinematics $E_{e}=705.11 \mathrm{MeV}$ and $\theta_{e}$ $=42.6^{\circ}$. 


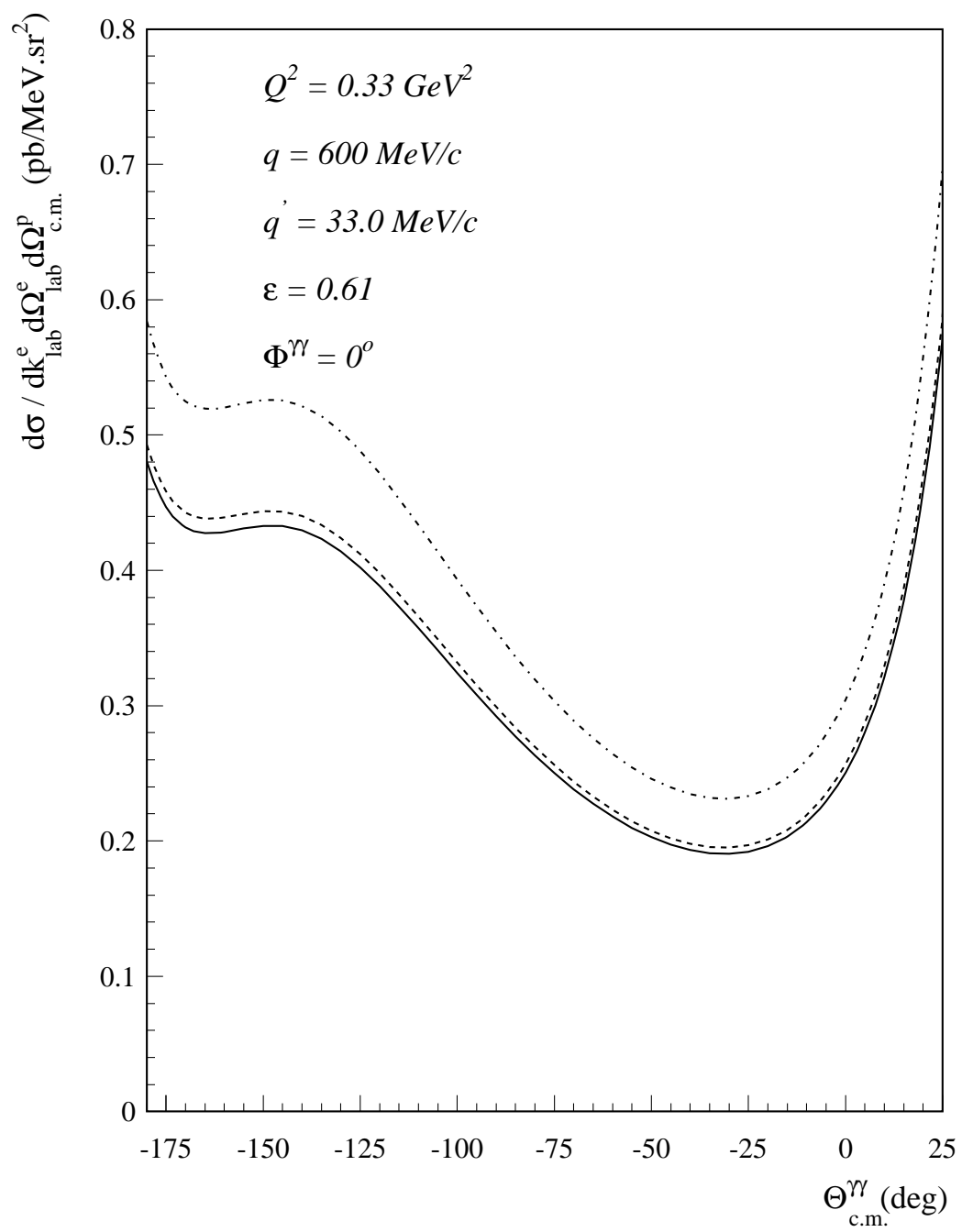

FIG. 13. Differential $e p \rightarrow e p \gamma$ cross section for MAMI kinematics at $q^{\prime}=33 \mathrm{MeV} / \mathrm{c}$. Dashed-dotted curve : $\mathrm{BH}+$ Born contribution, dashed curve : $\mathrm{BH}+$ Born + virtual radiative correction, full curve : $\mathrm{BH}+$ Born + total radiative correction. The real radiative correction is shown here for a maximal soft-photon energy of $\Delta E_{s}=10 \mathrm{MeV}$. 


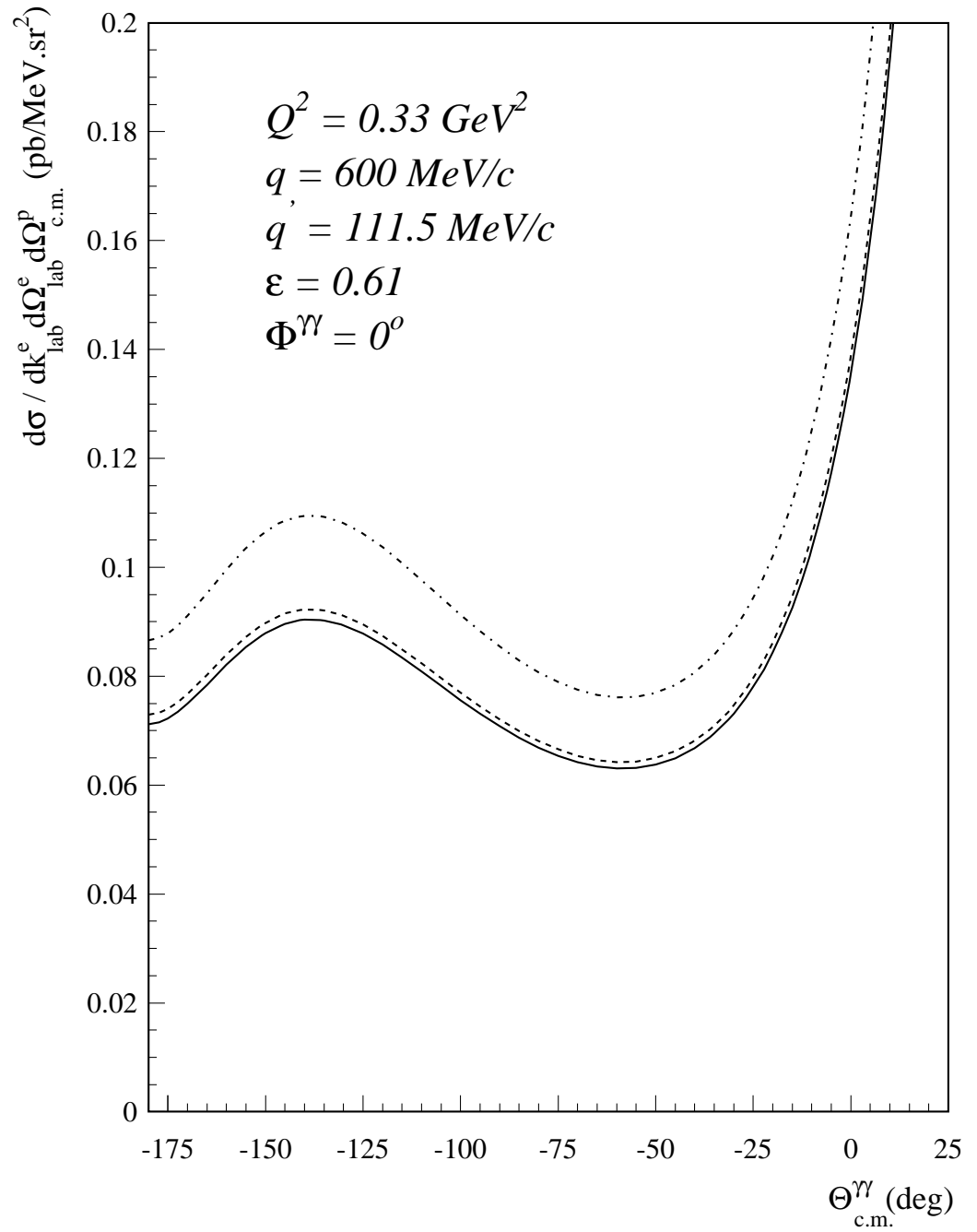

FIG. 14. Differential $e p \rightarrow e p \gamma$ cross section for MAMI kinematics at $q^{\prime}=111.5 \mathrm{MeV} / \mathrm{c}$. Curve conventions as in Fig.13. The real radiative correction is shown here for a maximal soft-photon energy of $\Delta E_{s}=10 \mathrm{MeV}$. 


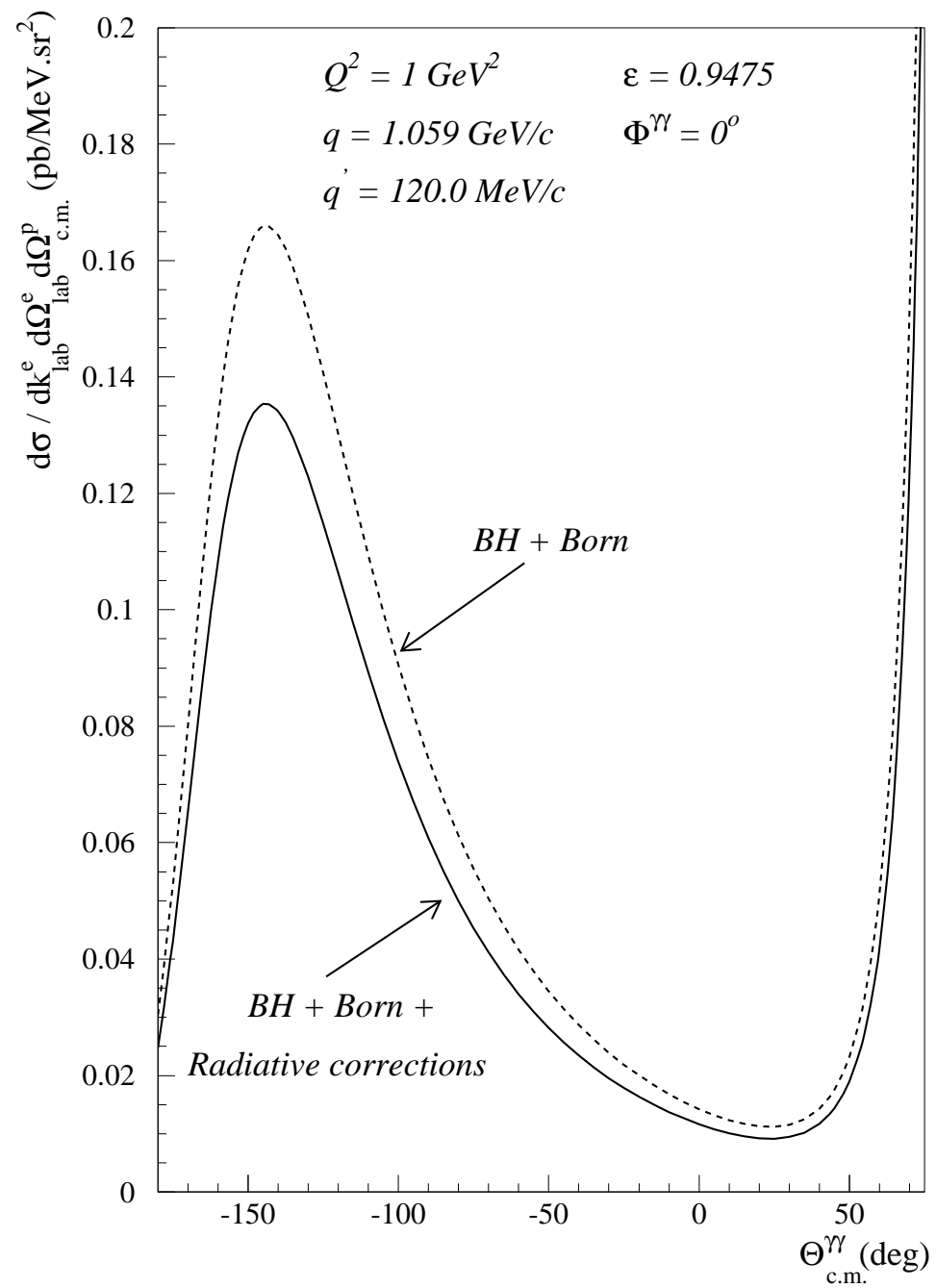

FIG. 15. Differential $e p \rightarrow e p \gamma$ cross section for JLab kinematics at $q^{\prime}=120 \mathrm{MeV} / \mathrm{c}$. The BH + Born result is compared with the result including virtual radiative corrections. 


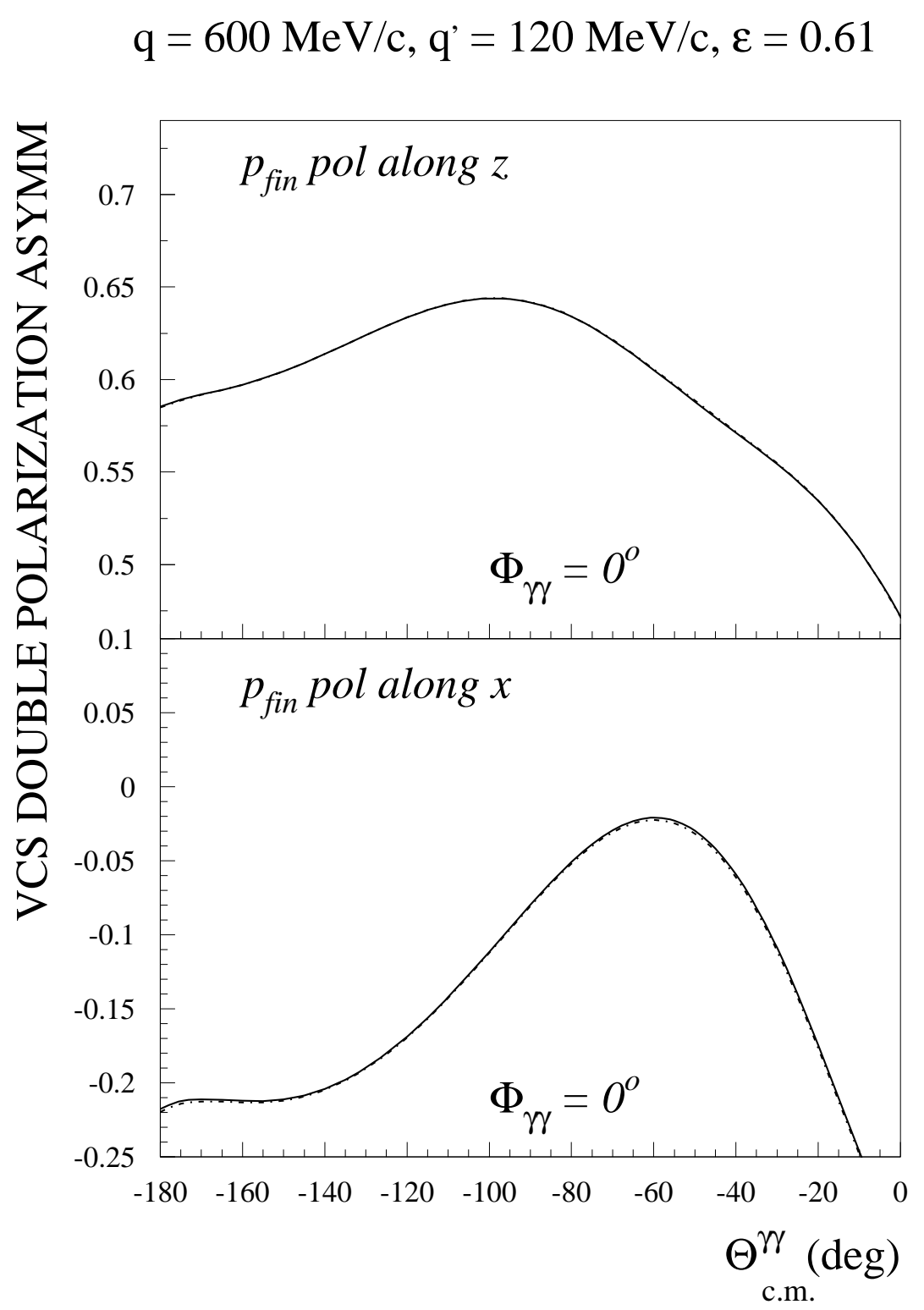

FIG. 16. Double polarization asymmetry for VCS with proton polarized along the z-axis (upper panel) or polarized along the x-axis (lower panel) for MAMI kinematics. Dashed-dotted curve : $\mathrm{BH}+$ Born, full curve $: \mathrm{BH}+$ Born + radiative corrections (both curves nearly coincide!). 


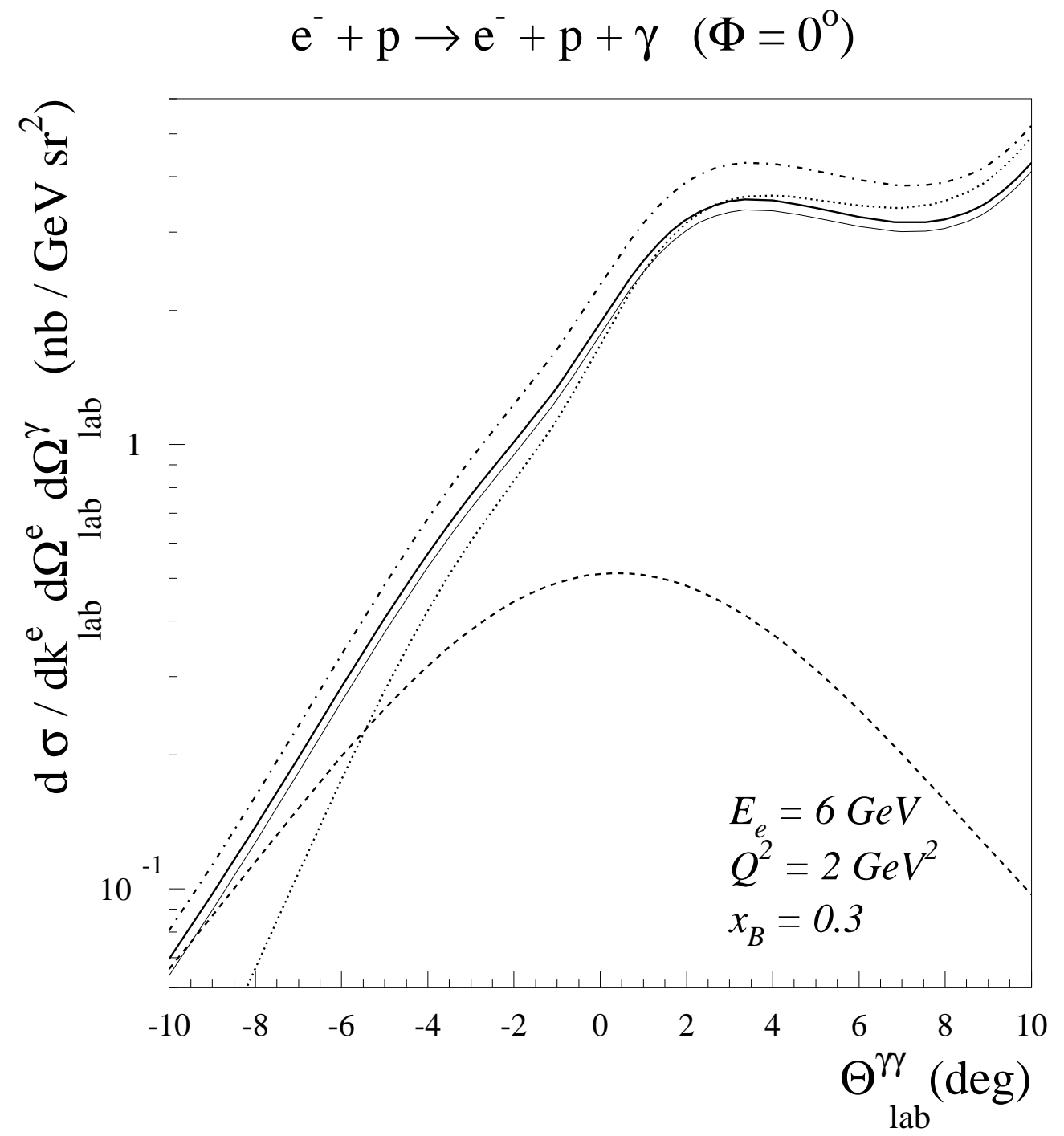

FIG. 17. Differential ep $\rightarrow e p \gamma$ cross section in lab: DVCS kinematics at JLab. Dotted curve : BH, dashed curve : DVCS, dashed-dotted curve : BH + DVCS, thin full curve : BH + DVCS + virtual radiative corrections. The thick full curve represents the $\mathrm{BH}+$ DVCS + virtual and real radiative corrections, where the real radiative corrections are calculated with $\Delta E_{s}=0.1 \mathrm{GeV}$, which corresponds with a cut in the missing mass spectrum (Eq. (59)) of $M_{m 1}^{2}-M_{N}^{2} \simeq 0.21 \mathrm{GeV}^{2}$. 


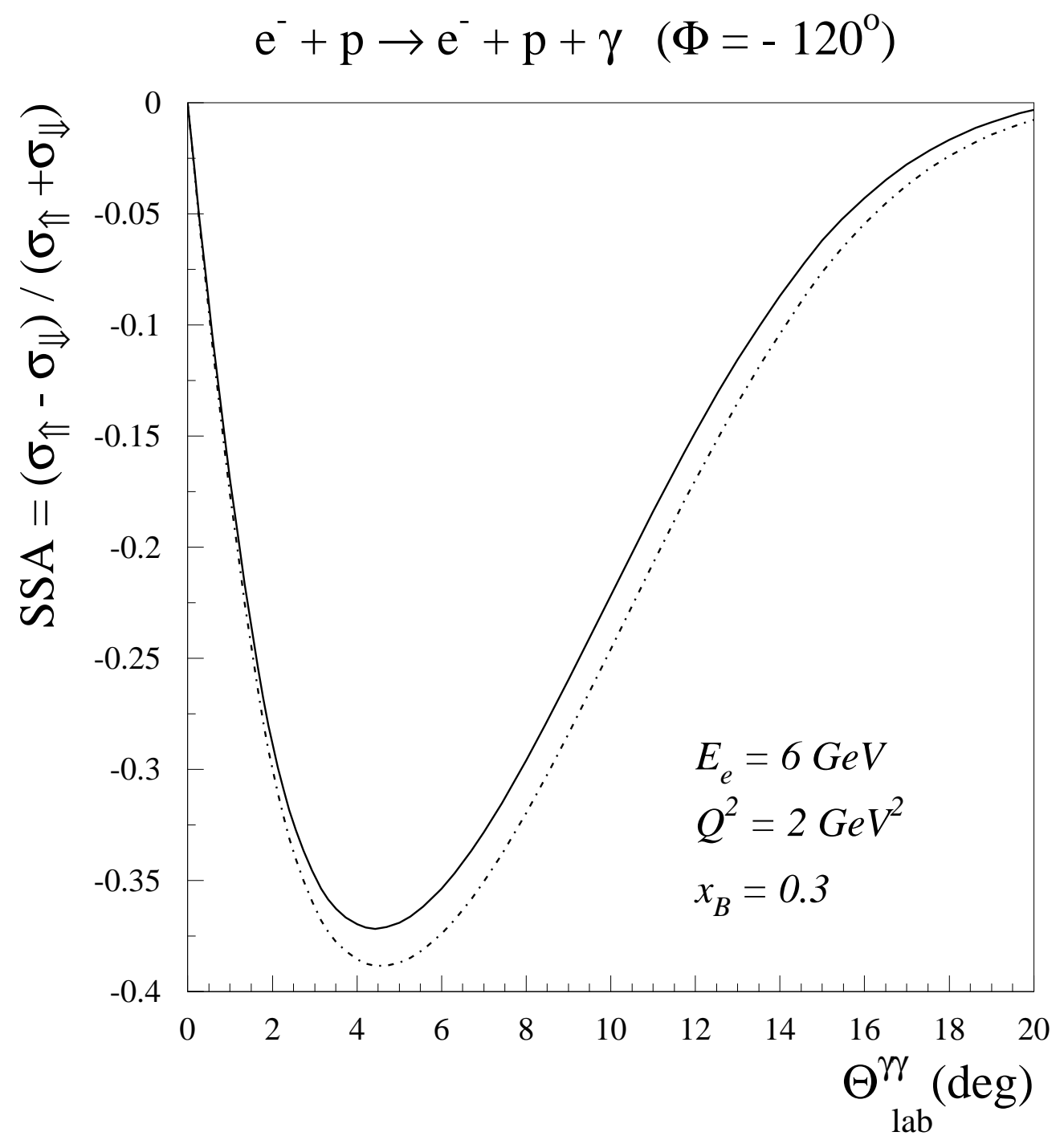

FIG. 18. Electron single spin asymmetry : DVCS kinematics at JLab. Dashed-dotted curve : $\mathrm{BH}+\mathrm{DVCS}$, full curve $: \mathrm{BH}+\mathrm{DVCS}+$ radiative corrections. 

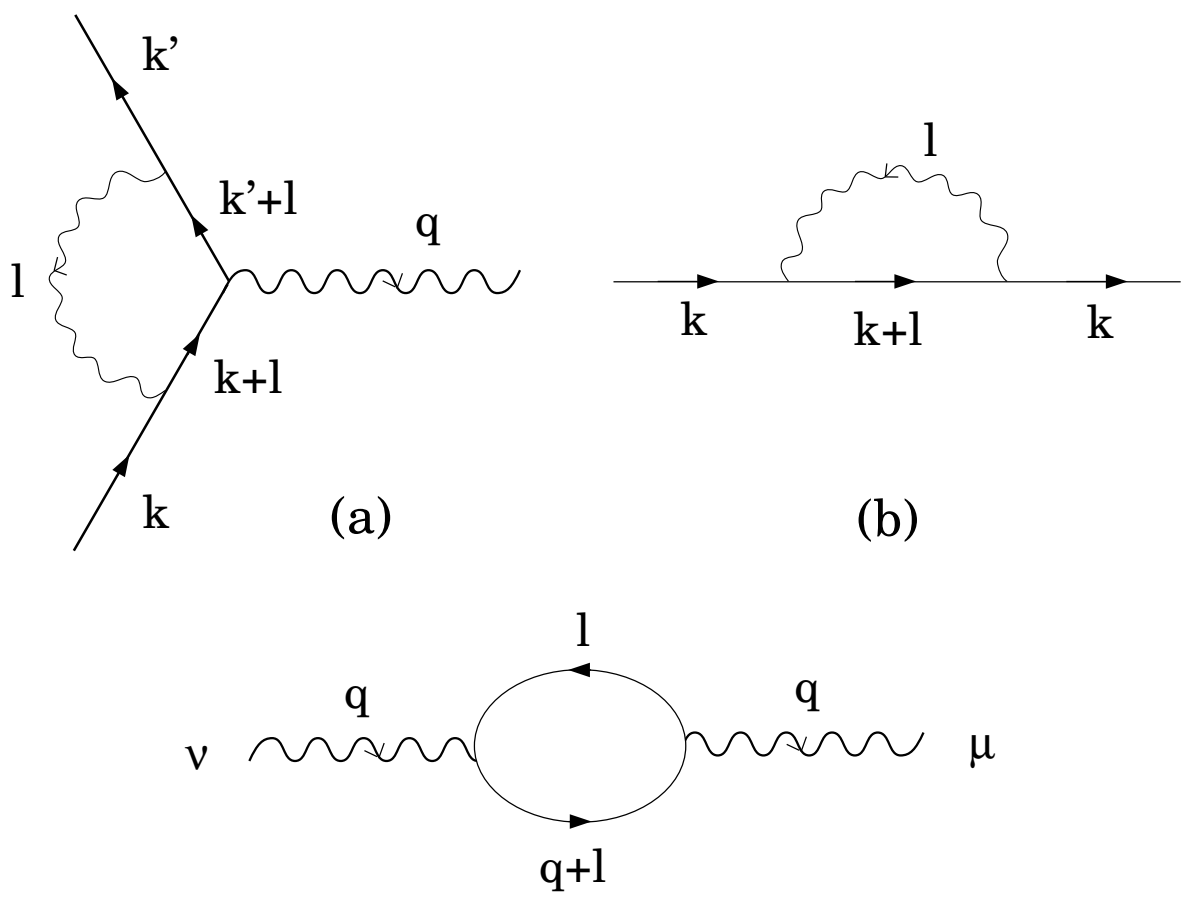

(c)

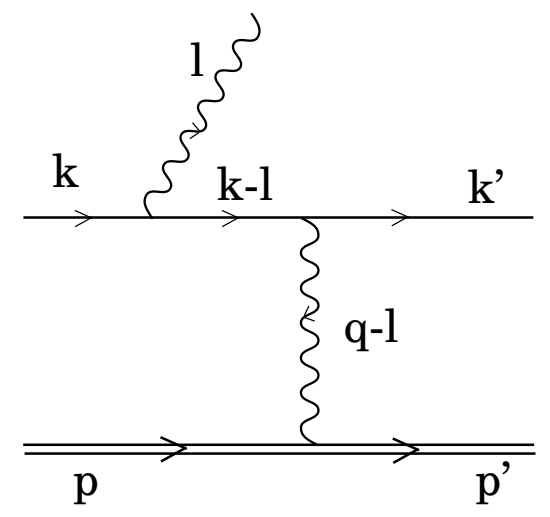

(d)

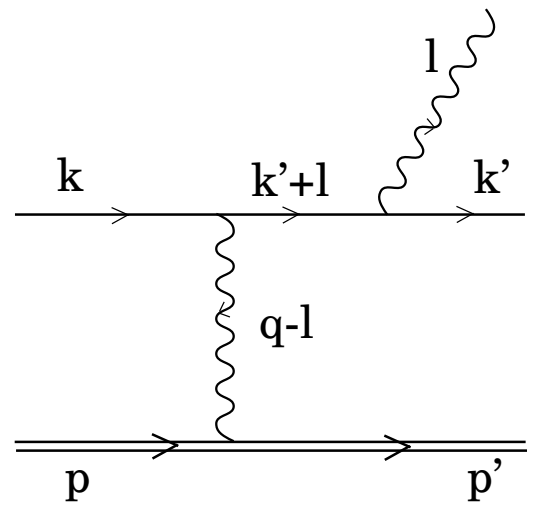

(e)

FIG. 19. First order virtual and real radiative correction processes : (a) vertex diagram, (b) lepton self energy diagram, (c) photon polarization diagram, (d) and (e) soft-photon emission contributions to elastic lepton-nucleon scattering. 